FORSCHUNGSERGEBNISSE DER WIRTSCHAFTSUNIVERSITÄT WIEN

Dagmar Kiefer

\title{
Multicultural Work in Five United Nations Organisations
}




\section{Dagmar Kiefer}

\section{Multicultural Work in Five United Nations Organisations}

This book aims at developing an understanding of multicultural work in five Organisations of the United Nations. The context of international organisations differs from international companies, amongst others, with regards to the legal base, the financial and operational agenda, and human resource practices.

The purpose of this study is to explore which factors are a trigger, a barrier, and/ or an influence on team and group work in this specific context. Based on 50 semi-structured, personal interviews which were analysed within the framework of constant comparison method, a typology of multicultural work is provided. Moreover, this study offers an integrative view of the reciprocal relationships between organisation, management, group and individual.

Dagmar Kiefer received her doctorate in International Management from the Vienna University of Economics and Business in 2008. During her doctoral studies she mostly worked full time for an international company and spent numerous months abroad to work and to research. 
Multicultural Work in Five Nations Organisations 


\section{Forschungsergebnisse der Wirtschaftsuniversität Wien}

\section{Band 35}

\section{施

Frankfurt am Main · Berlin · Bern · Bruxelles · New York · Oxford · Wien 
Dagmar Kiefer

\section{Multicultural Work in Five United Nations Organisations}

An Austrian Perspective

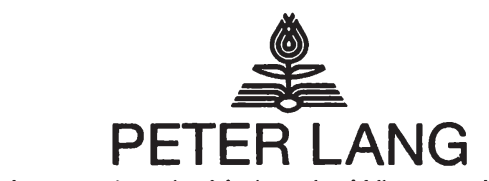

Internationaler Verlag der Wissenschaften 


\title{
Bibliographic Information published by the Deutsche
} Nationalbibliothek

The Deutsche Nationalbibliothek lists this publication in the Deutsche Nationalbibliografie; detailed bibliographic data is available in the internet at <http://www.d-nb.de>.

Open Access: The online version of this publication is published on www.peterlang.com and www.econstor.eu under the international Creative Commons License CC-BY 4.0. Learn more on how you can use and share this work: http://creativecommons. org/licenses/by/4.0.

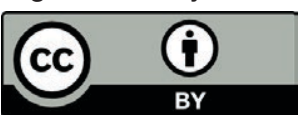

This book is available Open Access thanks to the kind support of ZBW - Leibniz-Informationszentrum Wirtschaft.

\section{Sponsored by the Vienna University of Economics and Business Administration.}

Cover design:

Atelier Platen according to a design of the advertising agency Publique. University logo of the Vienna University of Economics and Business Administration.

Printed with kindly permission of the University.

\author{
ISSN 1613-3056 \\ ISBN 978-3-631-59170-3 \\ ISBN 978-3-631-75389-7 (eBook) \\ (c) Peter Lang GmbH \\ Internationaler Verlag der Wissenschaften \\ Frankfurt am Main 2009 \\ All rights reserved.
}

All parts of this publication are protected by copyright. Any utilisation outside the strict limits of the copyright law, without the permission of the publisher, is forbidden and liable to prosecution. This applies in particular to reproductions, translations, microfilming, and storage and processing in electronic retrieval systems.

www.peterlang.de 
To my family and friends

Dagmar Kiefer - 978-3-631-75389-7

Downloaded from PubFactory at 01/11/2019 05:36:21AM

via free access 
Dagmar Kiefer - 978-3-631-75389-7

Downloaded from PubFactory at 01/11/2019 05:36:21AM

via free access 


\begin{abstract}
This book aims at developing an understanding of multicultural work in five Organisations of the United Nations. The context of international organisations differs from international companies, amongst others, with regards to the legal base, the financial and operational agenda, and human resource practices.

The purpose of this study is to explore which factors are a trigger, a barrier, and/or an influence on team and group work in this specific context. Based on 50 semi-structured, personal interviews a typology of multicultural work is provided. Moreover, this study offers an integrative view of the reciprocal relationships between organisation, management, group and individual.
\end{abstract}

\title{
Acknowledgments
}

This book is based on the results of an OeNB Jubiläumsfonds research project (Project Number 11618) and my doctoral thesis, submitted to the Vienna University of Economics and Business Administration in February 2008.

Cordial thanks are due to my interview partners in the United Nations. This book would not have been possible without their cooperation and willingness to support my research. Many thanks for helpful advice and discussion of related isssues are due to Anne-Katrin Neyer.

I especially would like to thank my supervisor Univ. Prof. Dr. Gerhard Fink for his valuable scientific support and the time invested during my doctoral studies, and also the second reviewer of my thesis, Univ. Prof.in. Dr.in. Edeltraud Hanappi-Egger for her constructive feedback in the final stage of writing up my doctoral thesis.

Last but not least a special thank you to my family and my friends for their endless encouragement not just for this project but in life. 
Dagmar Kiefer - 978-3-631-75389-7

Downloaded from PubFactory at 01/11/2019 05:36:21AM

via free access 


\section{Table of Contents}

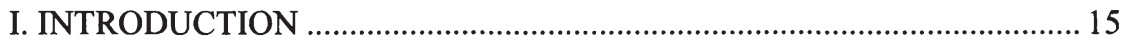

1.1. Background to the research ...................................................................... 15

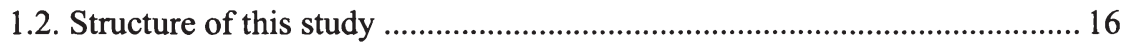

II. LITERATURE REVIEW ON CURRENT STUDIES INTO TEAMS AND GROUPS IN ORGANISATIONS ....................................................... 18

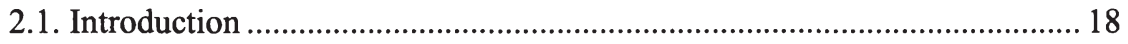

2.2. Definition of the terms team and group .................................................. 18

2.3. Journal review: Studies of teams and groups in real organisational

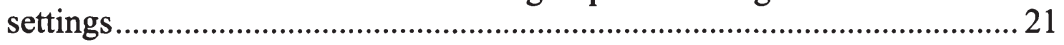

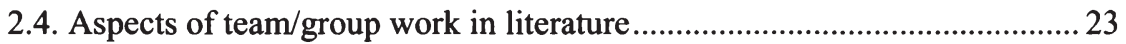

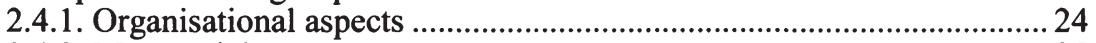

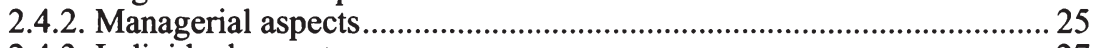

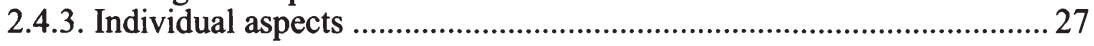

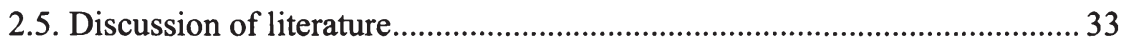

III. RESEARCH METHODOLOGY, METHOD, RESEARCH DESIGN

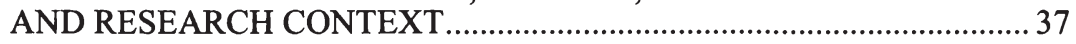

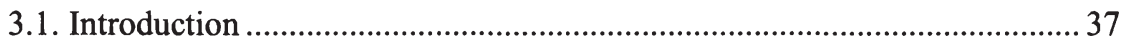

3.2. Methodology: Grounded Theory ............................................................... 37

3.2.2. Method - Case Study .............................................................................. 38

3.2.3. Objectivity, reliability, validity, and generalisability ............................. 39

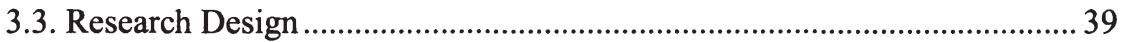

3.3.1. Semi-structured interviews .................................................................. 40

3.3.2. Data Analysis - Constant Comparative Method ..................................... 44

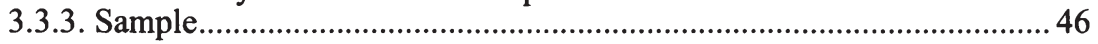

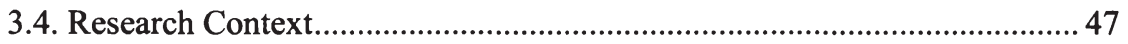

IV. EMPIRICAL FINDINGS - DATA ANALYSIS ....................................... 52

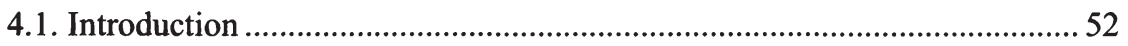

4.2. Team or group work? A typology grounded in the data ............................. 52

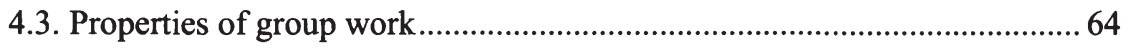




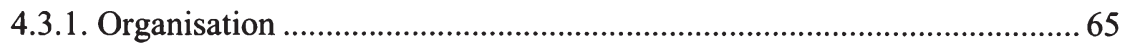

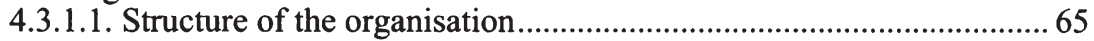

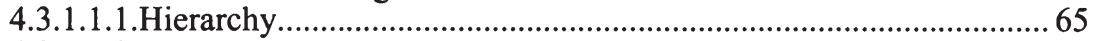

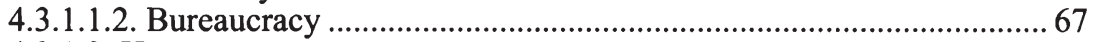

4.3.1.2. Human resource management ........................................................ 72

4.3.1.3. Moderator: Boundary crossing and boundary spanning ....................... 77

4.3.1.3.1. Individual boundary spanning ........................................................ 79

4.3.1.3.2. Organisational boundary spanning ................................................ 80

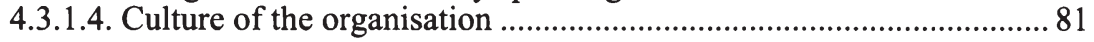

4.3.2. Employee - Management relationship .................................................... 84

4.3.2.1. Management - the crucial link .......................................................... 84

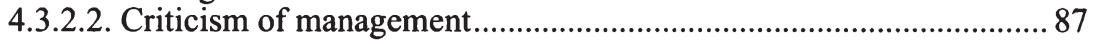

4.3.3. Employee - Co-worker relationship .......................................................92 92

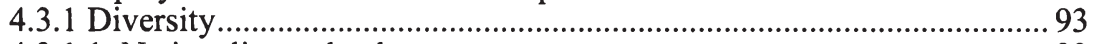

4.3.1.1. Nationality and culture ....................................................................... 93

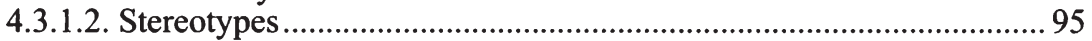

4.3.1.2.1. Excursus: Working in the field ..................................................... 96

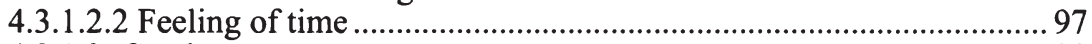

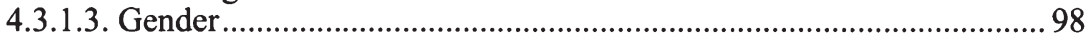

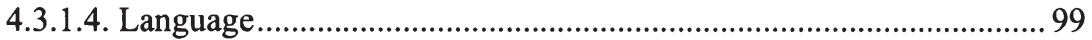

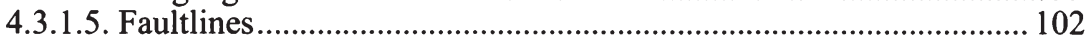

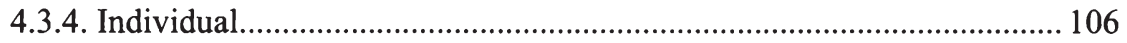

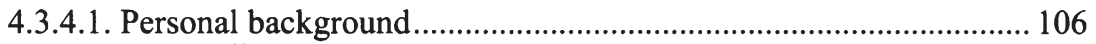

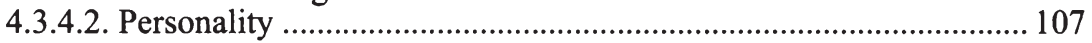

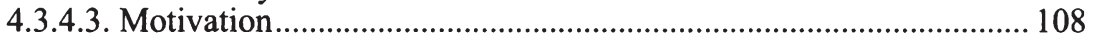

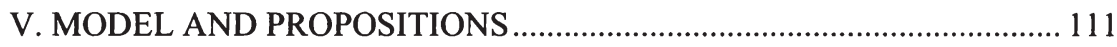

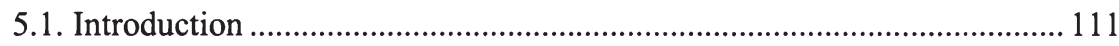

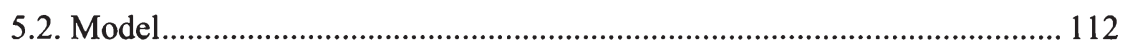

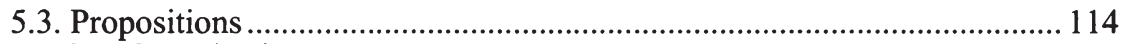

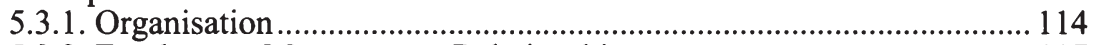

5.3.2. Employee - Management Relationship.............................................. 117

5.3.3. Employee - Co-worker Relationship.................................................. 118

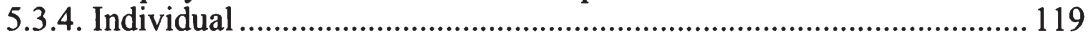

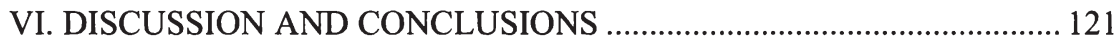

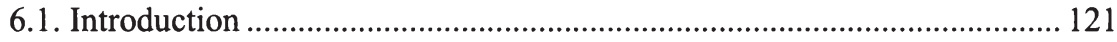

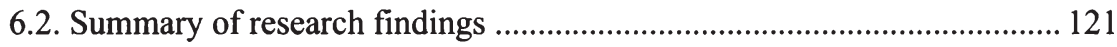

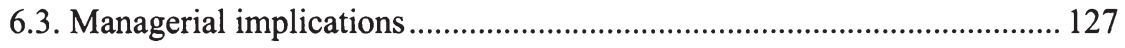

6.4. Limitations and directions for future research .................................... 132 
VII. BIBLIOGRAPHY

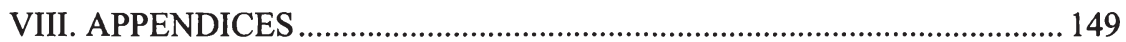

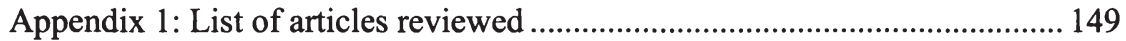

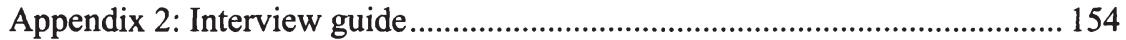

Appendix 3: Notes on interview situation........................................................ 155 


\section{List of abbreviations}

D-Staff Directors Staff

ECE Economic Commission for Europe

GA General Assembly

G-Staff General Services Staff

HRM Human Resource Management

IAEA International Atomic Energy Agency

ILO International Labour Organisation

JPO Junior Professional Officer

OCHA Office for the Coordination of Humanitarian Affairs

OIOS Office of Internal Oversight Services

P-Staff Professional Staff

SG Secretary General

SC Security Council

UN United Nations

UN Charter United Nations Charter

UNDP United Nations Development Programme

UNIDO United Nations Industrial Development Organisation

UNO United Nations Organisation

UNOPS United Nations Office for Project Services

USG Under Secretary General

WIPO World Intellectual Property Organisation 


\section{List of figures}

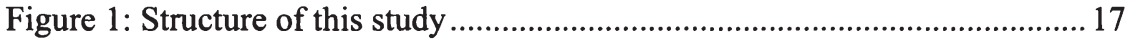

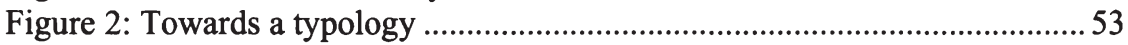

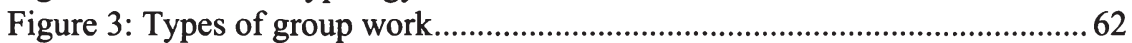

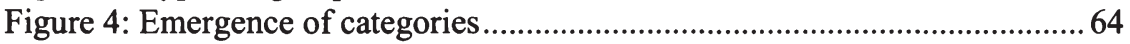

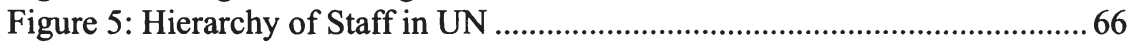

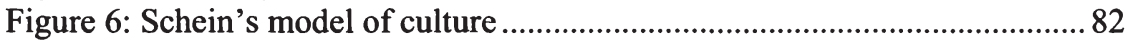

Figure 7: Network view on organisational and managerial components............91

Figure 8: Network view on employee-Co-worker relationship .......................... 93

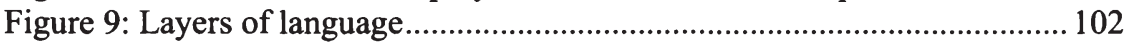

Figure 10: Network view on components of group work ................................. 110

Figure 11: A conceptual model of group work in five UN Organisations ....... 113

Figure 12: Five managerial implications...................................................... 131 


\section{List of tables}

Table 1: Definitions of team/group in chronological order ............................... 19

Table 2: Journal screen from 1998 until March 2007 .......................................... 22

Table 3: Demographics of the sample ............................................................4 47

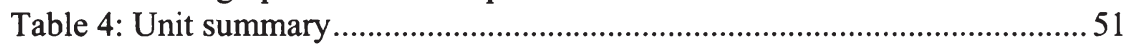

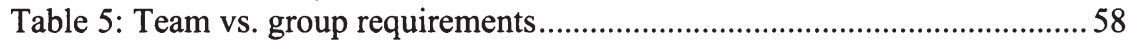

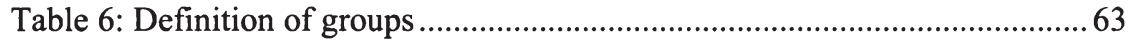

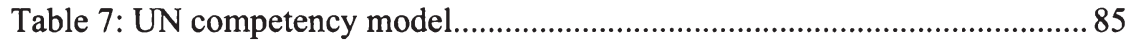

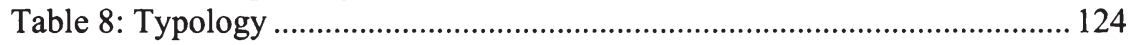




\section{Introduction}

\subsection{Background to the research}

The United Nations Organisation at the beginning of the $21^{\text {st }}$ century is different from the Organisation which emerged more than 60 years ago. The number of member states has increased from 51 founding members in 1945 to 191 up to day. The United Nations Organisation has grown from a group of countries which have committed themselves voluntarily to safeguard peace, to an international organisation with several headquarters and a wide range of missions around the globe. This world organisation works with all different kinds of partners (governments, other international organisations, civil society groups, private sector companies etc.) on equally different activities. Consequently, the United Nations Organisation is a work place for a constantly growing number of people who come from different parts of the world, belonging to different cultures and religions.

Simultaneously the criticism of this world organisation has increased. Today's United Nations Organisation is considered being lame, inefficient, and costly. A legitimate question therefore is whether or not the structure of this organisation and its staff can keep up with the new needs and requirements induced by a changing environment?

Numerous attempts have been made to reform the United Nations Organisations. The latest reform proposal was initiated by the former Secretary-General, Kofi Annan (2006), who suggested reforms in six areas: people, leadership, information and communications technology, delivering services, budget and finance, and governance. The detailed measures formulated in the proposal aimed to enable the organisation to make better use of its managerial and human resources. Interestingly, Mr. Annan mentioned a reform of the personnel system on the first place suggesting reforms in recruitment and career development. These suggestions also alluded to a reform at the management level and of the leadership situation.

Unfortunately this reform package was not passed because the majority of the member states voted against it. This vote shows the fragile side of this huge intergovernmental organisation which is financed by states and therefore the very same states have the power to induce change, or block it. As formulated by one interviewee: „The UN is very weak, it is a receiver of political and economical realities."

However, this does not mean that there is no remedy whatsoever.

In management literature, the importance of teams and groups is accentuated as a factor increasing the performance of organisations as well as the performance 
of individuals. Numerous studies have shown that teams and groups decisively contribute to innovation, quality of service, cost reduction and increased productivity. Whilst a lot of research into teams and groups in internationally operating companies has been conducted, only rudimentary empirical research into the functioning of teams and groups in international organisations has been undertaken so far. Consequently, the contribution of this research is to assess the state of the art of team and group work in management literature and at the same time to investigate what kind of group and/or team work is practised in five Organisations of the United Nations. This study then attempts to highlight triggers and barriers for team and group work in five Organisations of the United Nations and aims at developing propositions and a model of analysis specifically addressing these triggers and barriers.

Summarising, the two central research questions are:

- What kind of team and group work exists in five United Nations Organisations?

- Which factors are a trigger, a barrier, and/or influence team and group work in this context?

Answering these two questions leads to develop:

- Propositions comprising the organisational, group and individual context

- A model of analysis

- Managerial implications

The type of question (how and why team and group work is/is not practised in five United Nations Organisations), the explanatory purpose of this study (e.g. the identification of triggers and barriers for team and group work) and the focus on contemporary events led me to apply a qualitative research design. $50 \mathrm{semi}$ structured interviews with predominately Austrian staff in five Organisations of the United Nations based in three locations were conducted for this case study. The interview data was analysed using the constant comparison method developed by Glaser \& Strauss (1967) and as explicated by Boeije (2002).

\subsection{Structure of this study}

This study is structured along the following chapters:

Chapter II provides an overview of management literature referring to team and group work and discusses in a critical review the controversial findings. The salience of missing definitions in literature is the main point of criticism. Chapter III covers the research methodology, method and explains the research design. 
This chapter also discusses the sample and the context of research describing the five Organisations under investigation. Chapter IV presents empirical findings based on the analysis of 50 semi-structured interviews in five Organisations of the United Nations. Based on the empirical findings a theoretical model of triggers and barriers for team work is presented in chapter V. This study concludes with chapter VI which raises a discussion of research findings, managerial implications and limitations of this study.

Figure 1: Structure of this study

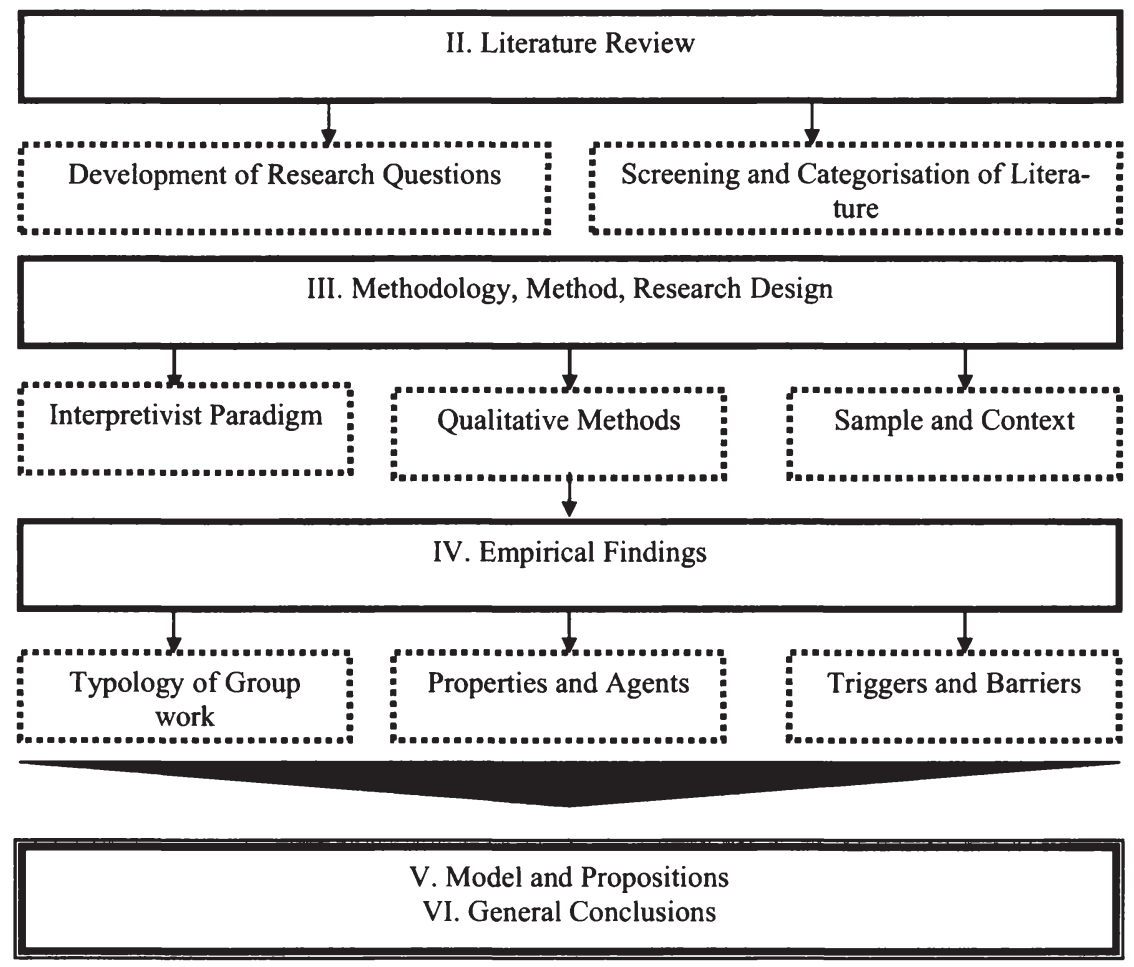

Source: author 


\section{Literature Review on current Studies into Teams and Groups in Organisations}

\subsection{Introduction}

Teams and groups in companies have been intensively studied over the past two decades. Different disciplines looked at various aspects of teams and groups in the private sector. To list a few, research has investigated group composition, task design, organisational context, environmental factors, internal processes, personality traits, diversity and effectiveness. Furthermore, the definitions of teams and groups used in the different studies vary. Existing literature has investigated work teams, work groups, self managed teams, management teams, and most recently virtual teams. In other words: the body of literature is vast.

The following literature review systematically structures existing team and group literature relevant to the conceptual framework of this thesis.

The aim of this review is

- to give an overview of the definitions of the terms team and group

- to give an overview of studies of teams and groups in real organisational settings and

- to identify which factors enhance and influence teamwork

\subsection{Definition of the terms team and group}

As mentioned above, a variety of definitions for the terms team and group exist and often the terms are used interchangeably. The mere existence of two terms suggests that there must be a difference. The Oxford English Dictionary (1989) records the following uses of the terms:

Group (in general): A number of persons or things regarded as forming a unit on account of any kind of mutual or common relation, or classified together on account of a common degree of similarity (Vol. VI, p 887)

Team: A number of persons associated with some joint action; now esp. a definite number of persons forming a side in a match, in any team sport, hence a group collaborating in their professional work or in some enterprise or assignment (Vol. XVII, p 692)

According to the dictionary the term group is the more general one whereas the term team is more special as joint action is implied. Hare (1992) argues that the term team is reserved for work groups that are highly visible and require more differentiated roles and more integration of members' activity. He further argues that no clear distinction between the terms is evident in the social-psychological literature. The current literature review also shows that researchers often use the terms interchangeably: for example Bettenhausen (1991) intensively reviewed group research and only refers to teams in the context of team building activi- 
ties, whereas Cohen and Bailey (1997) refer to Bettenhausen's work as a review of teams. Over the years, the term team has largely replaced the term group. Especially in management literature the term team is used more often (e.g. empowered teams, self managed teams, team effectiveness) whereas the academic literature used the word group more frequently (group dynamics, group cohesion, group effectiveness). Still there is no universally accepted definition of the terms in management literature (Guzzo \& Dickson, 1996). However, some researchers agreed on one widely established definition of team being "a collection of individuals who are interdependent in their task and who share responsibility for outcomes" (Guzzo \& Shea, 1992; Hackman, 1987; Sundstrom, DeMeuse, \& Futrell, 1990).

Table 1 below gives an overview of the definitions of the terms team and group found in the articles analysed.

Table 1: Definitions of team/group in chronological order

\begin{tabular}{|l|l|}
\hline Author & Definition \\
\hline $\begin{array}{l}\text { Francis \& } \\
\text { Young } \\
(1979)\end{array}$ & $\begin{array}{l}\text { Occasionally we meet an exceptional group that combines high } \\
\text { morale, effective task performance, and clear relevance to the } \\
\text { organisation and we award the accolade of a 'team'. }\end{array}$ \\
\hline $\begin{array}{l}\text { Hackmann } \\
(1983)\end{array}$ & $\begin{array}{l}\text { Work groups are intact, bounded social systems, with interde- } \\
\text { pendent members and differentiated member roles that pursue } \\
\text { shared, measurable goals. They consist of all employees who } \\
\text { report to the same supervisor and who are engaged in tasks re- } \\
\text { quiring some degree of coordination. }\end{array}$ \\
\hline $\begin{array}{l}\text { Wall, Kemp, } \\
\text { Clegg (1986) }\end{array}$ & $\begin{array}{l}\text { Self-directed work teams are those organizational units in } \\
\text { which (a) employees share functionally interrelated tasks and } \\
\text { are collectively responsible for end products, (b) individual } \\
\text { team members have the variety of skills necessary to perform } \\
\text { tasks that are the collective responsibility of the team, and (c) } \\
\text { employees receive feedback and evaluations that are given in } \\
\text { terms of team performance. Self-directed work teams have a } \\
\text { high degree of self-determination that includes control over the } \\
\text { pace of work, distribution of tasks, work breaks, and participa- } \\
\text { tion in recruiting and training new members. }\end{array}$ \\
\hline $\begin{array}{l}\text { Dyer (1987) } \\
\text { A team is a collection of people who must collaborate, to some } \\
\text { degree, to achieve common goals. }\end{array}$ \\
\hline $\begin{array}{l}\text { Patten } \\
\text { (1988) }\end{array}$ & $\begin{array}{l}\text { The group must have a natural reason for working together. } \\
\text { each others experience, abilities, and commitment in order to } \\
\text { fulfil mutual objectives. Group members must be committed to }\end{array}$ \\
\hline
\end{tabular}




\begin{tabular}{|c|c|}
\hline & $\begin{array}{l}\text { the idea that working together as a group leads to more effec- } \\
\text { tive decisions. The group must be accountable as a functioning } \\
\text { unit within a larger organisation. }\end{array}$ \\
\hline $\begin{array}{l}\text { Alderfer } \\
(1977), \\
\text { Hackman } \\
\text { (1987), } \\
\text { Sundstrom, } \\
\text { DeMeuse, \& } \\
\text { Futrell } \\
(1990)\end{array}$ & $\begin{array}{l}\text { A team is a collection of individuals who are interdependent in } \\
\text { their task, who share responsibility for outcomes, who see } \\
\text { themselves and who are seen by others as an intact social entity } \\
\text { embedded in one or more lager social systems (for example } \\
\text { business unit or the corporation), and who manage their rela- } \\
\text { tionships across organisational boundaries. }\end{array}$ \\
\hline $\begin{array}{l}\text { Orsburn, } \\
\text { Moran, Mus- } \\
\text { selwhite, } \\
\text { Zenger, \& } \\
\text { Perrin (1990) }\end{array}$ & $\begin{array}{l}\text { Successful SMWTs (Self managing work teams) have the } \\
\text { proper resources at their control, a wide range of cross- } \\
\text { functional skills among the team members, and all the informa- } \\
\text { tion they need to make sound decisions. Additionally, SMWTs } \\
\text { have the authority to plan, set priorities, coordinate with other } \\
\text { teams, measure progress, and take corrective actions. }\end{array}$ \\
\hline $\begin{array}{l}\text { Salas, Dick- } \\
\text { inson, Con- } \\
\text { verse \& } \\
\text { Tannenbaum } \\
\text { (1992) }\end{array}$ & $\begin{array}{l}\text { Contrary to groups, teams consist of two or more individuals } \\
\text { that can be characterized by high task interdependence, high } \\
\text { role differentiation, high task differentiation, and distributed } \\
\text { expertise. }\end{array}$ \\
\hline $\begin{array}{l}\text { Guzzo \& } \\
\text { Dickson } \\
\text { (1996) based } \\
\text { on Alderfer } \\
\text { and Hack- } \\
\text { mann }\end{array}$ & $\begin{array}{l}\text { A workgroup is made up of individuals who see themselves } \\
\text { and who are seen by others as a social entity, who are interde- } \\
\text { pendent because of the tasks they perform as members of a } \\
\text { group, who are embedded in one or more larger social systems } \\
\text { (eg. community, organisation), and who perform tasks that af- } \\
\text { fect others (such as customers or co-workers). }\end{array}$ \\
\hline $\begin{array}{l}\text { Corderly } \\
(2003), \text { For- } \\
\text { syth (1999) }\end{array}$ & $\begin{array}{l}\text { Teams are commonly regarded as structured sets of people that } \\
\text { pursue collective performance objectives within larger organ- } \\
\text { izational systems and that require coordinated interactions to } \\
\text { successfully accomplish relevant tasks. }\end{array}$ \\
\hline $\begin{array}{l}\text { McCreery \& } \\
\text { Bloom } \\
(2000)\end{array}$ & $\begin{array}{l}\text { A self-managing work team (SMWT) is typically given a wide } \\
\text { latitude in decision making in organizations. }\end{array}$ \\
\hline $\begin{array}{l}\text { Marquardt \& } \\
\text { Horvath } \\
(2001)\end{array}$ & $\begin{array}{l}\text { Multicultural teams are task-oriented groups consisting of peo- } \\
\text { ple of different national cultures. }\end{array}$ \\
\hline
\end{tabular}


The definitions above bear several messages:

At a general level, most definitions include (1) who is on the team/group, (2) why those individuals are on the team/group and (3) what their task and/or goal is. At a more narrow level some definitions describe specific types of teams/groups only, whereas others are less detailed and structured. Nonetheless, the general idea of a team or a group is basically the same which may be one reason for the interchangeable use of the two terms. Contrary, Gibson \& Zellmer-Bruhn (2001) argue that in a multinational context the differences in the definition of teams account for different outcomes of similar studies.

Summing up, there is no clear definition of and no clear line between the terms group and team. As a result, researchers use the terms interchangeably and therefore both terms were included in the keyword search for this literature review.

\subsection{Journal review: Studies of teams and groups in real organisational set- tings}

The broad field of team/group work and its outcomes has been widely discussed in academia in the past decades. Consequently, there is such a huge body of literature across various disciplines that in fact it has become rather difficult to get an overview of existing research. To give a structure to the findings of research into team and group work, the following steps have been taken:

A journal review of team and group literature in 25 academic journals and management journals between 1998 and March 2007 was conducted. The journals were chosen based on their rating (common consideration as top and leading academic journals) and because of their relevance in the field of group research and public administration.

Prior to the physical journal review a number of criteria for inclusion and exclusion of articles were developed. Only studies published in the journals listed (table 2) were included, only studies conducted in real work environments have been chosen as these findings can more readily be generalised to the world of work, and only studies of teams and/or groups of employees were included.

Consequently, the following exclusion criteria were developed: studies with students or experimental studies were not included because of the practical focus of the topic of this dissertation. Furthermore, management teams were excluded as this thesis focuses on employees. Lastly, action teams (e.g. sport teams), performing teams (e.g. musical groups), teams in hospitals (e.g. health care teams), and virtual teams were excluded. However, conceptual and theoretical articles as well as meta analyses have been looked at and will be presented in the review.

The literature review is partly based on three seminal reviews of team literature which have been conducted recently. Van Knippenberg \& Schippers (2007) reviewed literature on work group diversity from 1997-2005, Rasmussen \& Jeppe- 
sen (2006) assessed current knowledge of teams and associated psychological factors from 2000 to 2005, and Stock (2004) investigated drivers of team performance in articles published between 1990 and 2003. The current review includes all factors in one paper and covers the period between 1998 and March 2007. However, slightly different journals have been looked at and therefore not all articles listed in the reviews mentioned will be covered in this review.

A keyword search using the terms "team", "group", "work team", and "workgroup" within the journals listed was conducted. 96 out of approximately 12.000 articles in the 25 journals screened were identified as being relevant.

Table 2 gives an overview of the journals screened and the number of articles found relevant for the topic.

Table 2: Journal screen from 1998 until March 2007

\begin{tabular}{|l|c|}
\hline Journals screened & Relevant studies \\
\hline Academy of Management Journal & 14 \\
\hline Academy of Management Review & 4 \\
\hline Administrative Science Quarterly & 9 \\
\hline Applied Psychology & 3 \\
\hline $\begin{array}{l}\text { Asia Pacific Journal of Human Re- } \\
\text { sources }\end{array}$ & 0 \\
\hline European Management Journal & 1 \\
\hline Group Dynamics & 6 \\
\hline Human Relations & 3 \\
\hline $\begin{array}{l}\text { International Journal of Cross Cultural } \\
\text { Management }\end{array}$ & 0 \\
\hline $\begin{array}{l}\text { International Journal of Public Sector } \\
\text { Management }\end{array}$ & 0 \\
\hline $\begin{array}{l}\text { International Public Management Jour- } \\
\text { nal }\end{array}$ & 0 \\
\hline $\begin{array}{l}\text { International Studies of Management } \\
\text { and Organizations }\end{array}$ & 14 \\
\hline Journal of Applied Psychology & 1 \\
\hline Journal of Psychology & 1 \\
\hline Journal of Applied Social Psychology & 2 \\
\hline Journal of Business and Psychology & 0 \\
\hline Journal of International Management & 8 \\
\hline Journal of Management & 0 \\
\hline $\begin{array}{l}\text { Journal of Public Administration Re- } \\
\text { search and Theory }\end{array}$ & 10 \\
\hline Journal of Organisational Behavior & \\
\hline
\end{tabular}




\begin{tabular}{|l|c|}
\hline Journal of World Business & 6 \\
\hline Organisational Dynamics & 0 \\
\hline Personnel Psychology & 4 \\
\hline Public Administration Review & 0 \\
\hline Small Group Research & 9 \\
\hline Total & 96 \\
\hline
\end{tabular}

Subsequently, the 96 included studies were analysed by the author.

First, the articles were screened with regards to what kind of teams or groups were looked at to get an overview of the different terms used. Furthermore, the studies were grouped into quantitative and qualitative and I also distinguished between studies conducted in the private and the public sector.

In the following, a few general comments on the findings of the article search will be made before the literature analysis will be discussed in more detail.

- The majority of studies (73 studies out of 96) were conducted in the private sector and followed a quantitative research approach (76 studies). This phenomenon highlights the importance of qualitative research in the public sector and as a consequence the relevance of this dissertation. Nonetheless, the findings of existing studies form a vital basis for this research setting, as it is suggested that findings of team/group work in private sector companies can at least partly also be found in team/group settings in the public sector.

- Because of the variety of definitions and the interchangeable use of the terms, the articles identified were also analysed according to the definition of team/group and the terminology used in the different studies. The majority of articles ( 78 out of 96) did not provide a clear definition of the unit of analysis at all. Some authors used the terms team and group interchangeably and only 18 studies actually provided a definition of the units of interest.

Appendix 1 gives an overview of all articles screened. The articles are sorted by author in alphabetical order and labelled into categories such as private/public sector, qualitative/quantitative studies and show definitions of the terms used.

\subsection{Aspects of team/group work in literature}

In the following pages, the studies of teams and groups in organisational settings which have been found in the course of the journal and literature review will be presented in more detail.

The articles are grouped around three main aspects of interest in research into teams and groups: 
1. Organisational aspects such as organisational context, structure and culture,

2. Managerial aspects such as leadership, relationships between management and individuals, and

3. Individual aspects such as diversity in its various forms and personality.

Accordingly, the articles will be summarised and presented.

\subsubsection{Organisational aspects}

Organisational aspects such as the context in which groups and teams are embedded are depicted by the structure and culture of an organisation as well as society and politics. Context is inescapable because it is there whenever research is conducted. However, this does not mean that context is also considered in the setting and analysis of research. How much attention is paid to it lies solely in the hands of the researcher. Rousseau and Fried (2001, p.1) argue that the clue to more integrative studies is based in contextualisation which "entails linking observations to a set of relevant facts, events, or points of view that make possible research and theory that form part of a larger whole". In general, a rich description of the context of a research setting helps to explain the meaning, variation, and relationship among variables under study.

The current review showed that organisational aspects only play a peripheral role in group and team literature. Most studies seem to have neglected the importance of context and its effects on teams and groups.

Those contextual influences which received the most attention in research into teams and groups in multinational companies are, according to Jackson et al. (2003): task characteristics (e.g. Gilson, Mathieu, Shalley, \& Ruddy, 2005; Gilson \& Shalley, 2004; Pelled, Eisenhardt, \& Xin, 1999), organisational culture (e.g. Ely \& Thomas, 2001; Polzer, Milton, \& Swann, 2002), and strategic context (e.g. Jehn \& Bezrukova, 2004).

Few studies noted the importance of boundary spanning activities as a key variable that shapes group and team processes and outcomes. External activities or boundary spanning activities are directed toward the team's/ group's environment (other units within the same organisation, other organisations, or the general public).

Choi (2002) revealed that traditional rules and organisational structures are incomparable with team and group settings. On the one hand, tasks are too interdependent or too complex to be hierarchically managed. On the other hand, teams and groups need to communicate directly with other teams or units of the organisation or other organisations. For this reason, boundary activities on an organisational as well as on an individual level are being practised. 
Cross et. al (2000) demonstrated that adopting boundary-less organisational structures by implementing team and group structures lead to an increased need for boundary activities. In hierarchical structures boundary activities are executed by functional heads while they shift to team and group members in less formalised structures. Keller (2001) associated boundary activities with team and group performance and maintained that external activities lead to a decrease in cohesiveness of a team/group.

As mentioned in 2.3. only a few studies ( 4 out of 96) were conducted in the context of public organisations. Despite the fact that the studies were undertaken in public sector organisations, the authors did not provide a description of the context nor did they refer to a possible contextual impact on the results. This lack of contextualisation may impede the reader's understanding and interpretation of results.

In a longitudinal study Armstrong, Stassen, Wagar \& Cattaneo (2004) investigated job satisfaction in association with downsizing in a public sector environment. Members of work groups that remained intact during and after the downsizing reacted with lower levels of job satisfaction, lower job involvement and lower perceived justice than those who underwent a change of team membership during the downsizing. To assess the impact of the structure and culture of the organisation studied would have been an interesting avenue for research.

The same is true for the study conducted by Carless \& De Paola (2000) which focused on different forms of cohesion (task cohesion, social cohesion and individual attraction to the group) in a team setting.

Other studies conducted in the public sector investigated the role of perceived diversity in teams (Hobmann, Bordia, \& Gallois, 2003), and cognition and its indirect effects on team effectiveness (Rentsch \& Klimoski, 2001).

To summarise, a weakness of the studies analysed is that organisational aspects have often been ignored. If important contextual factors are ignored, results don't provide incremental knowledge. Yet, the understanding of the importance of contextual factors triggers one big problem: the number of potentially important aspects of context may quickly become overwhelming (see also Jackson et. al, 2003). It is totally impossible to capture all contextual factors in one study. Moreover, quantitative studies might be limited by a higher complexity whereas qualitative studies offer more space for inclusion of a wider range of contextual factors and as a result more comprehensible and integrative explanations.

\subsubsection{Managerial aspects}

The move towards team based structures in organisations challenges managers to lead and motivate not only individuals but teams/groups as a whole. There- 
fore, the behaviour of managers/leaders as a main driver of team/group performance definitely needs to be considered in theoretical foundations and practical studies. However, the literature review revealed that studies on team performance in general have overemphasized a direct link between demographic or functional characteristics and outcomes without adequately describing intervening psychological and social constructs. Literature focuses primarily on team processes but only rudimentary on the role of leadership styles and their impact on team performance (e.g. Zohar, 2002). As a result, the driving influence of managers on team processes and the role of leadership are still in an early stage of research.

Joshi \& Lazarova (2005) researched extensively into competences which leaders of teams/groups should possess of in order to effectively lead such teams/groups. Chang \& Tharenou (2004) tried to asses a set of competences needed to manage a multicultural work team/group. In a qualitative study managers as well as team/group members identified several competences which they considered as important: communication skills, the ability to continuously learn on the job and keep an interest in the work team/group, cultural empathy, honesty, and stability.

In a similar vein, Keller (2006) stressed in a longitudinal study that transformational leadership and initiating structure matter the most to R\&D team performance. A multilevel study of leadership, empowerment, and performance in teams was conducted by Chen, Kirkman, Kanfer, Allen \& Rosen (2007a). The study indicated that leaders need to use different approaches to motivate individual team members on the one hand and teams as a whole on the other hand. In the first case, a high level of mutual trust has to be built to empower individuals whereas in the latter case a delegation of autonomy and decision making is associated with high empowerment and performance. The study by Chen, Lam \& Zhong (2007b) showed that managers can motivate employees to improve performance by opening a clear and approachable channel through which they can seek positive and especially negative feedback.

Also most recently, Ambrosini, Bowman \& Burton-Taylor (2007) have investigated inter-team coordination activities as a driver of performance and a critical component of delivering customer satisfaction. Once again, the importance of encouraging communication in a team/group setting has been highlighted. Team empowerment as a trigger for higher performance and customer satisfaction was also reported by Kirkman \& Rosen (1999).

In a cross-cultural study, Chevrier (2003) observed that when managers encounter cross-cultural differences, they often ignore them and rely on individual team members to surmount these differences. Moreover, in multinational teams, team members reported that they tended to forget the nationality of their colleague and to focus on technical issues. A study by Reagans, Zuckermann \& McEvily (2004) showed that the demographic composition of an organisation limits man- 
agers to shape the demographic composition of a team. They argue that as a result the manager's interventions are less likely to be successful.

The relevant literature has arrived at the conclusion that managing people very much depends on contextual aspects. However, the weakness of most studies is that these contextual aspects have - if at all - only rudimentary been considered and described. A greater attention to the link between leadership and organisational context would broaden our understanding of the effects of different management styles.

Management practices and styles tend to be subject to trends and vary across cultures. Longitudinal studies would help to gain a deeper understanding of the complex interactions between individuals and to assess causality.

One example for cross-cultural, longitudinal research is the Global Leadership and Organisational Behaviour Effectiveness (GLOBE) research programme. The programme's focus lies on culture and leadership in 61 nations. House et al. (2004) found that regardless of culture, there are some universal aspects of leadership.

Research lacks studies of management in international organisations. While challenges in the private sector may be found in some form in the public sector, it appears that the public sector faces more political influences and has to deal with more rules and regulations (Feldman, 2005). This gap in literature is an interesting avenue for future research.

\subsubsection{Individual aspects}

Teams and groups are a collection of individuals who may be different with regards to a plethora of aspects such as nationality, gender, or personality. Hence, team and group literature is dominated by research into diversity in its various forms.

Diversity research

Typically diversity is conceptualised as a reference to the differences of personal attributes among interdependent members of a social unit (Jackson, 1992; Jackson et al., 2003; Triandis, Kurowski, \& Gelfand, 1994; Williams \& O'Reilly, 1998).

In practice diversity research focuses on differences

- in demographic compositions like age, ethnicity, gender

- in job related issues such as education, function, task, and

- between individuals with regards to personality, attitudes, and values. 
Williams \& O'Reilly (1998) covered 40 years of diversity research in their seminal research into work group diversity. They identified two main strands in research into work group diversity and performance:

- the social categorisation perspective and

- the information/decision-making perspective.

The social categorisation process is elicited by the assumption that demographic similarity/dissimilarity is the basis for categorising self and others into groups. As a result, people categorise demographically similar people as members of the in-group and dissimilar ones as members of the out-group (Brewer, 1979). Consequently, people of the in-group are more similar to one self than members of the out-group. Furthermore, people tend to trust members of the in-group more than members of the out-group (Brewer, 1979; Tajfel \& Turner, 1986). Overall the self categorisation perspective arrives at the conclusion that the more homogenous the team/group the higher the commitment of the members and thus the higher the performance.

The information/decision-making perspective holds the idea that heterogeneous teams/groups positively affect performance and creativity because of a broader range of perspectives, knowledge and abilities. This gives diverse teams/groups a larger pool of resources that may be especially helpful in dealing with nonroutine tasks and problems. In addition, diverse perspectives may lead to potentially more innovative ideas and to solutions which would not have been reached in a homogenous team/group (De Dreu \& West, 2001). However, some studies found a higher rate of conflict in heterogeneous teams/groups and a greater need for reconciliation between the members of the team/group (Jehn, Northcraft, \& Neale, 1999; Lovelace, Shapiro, \& Weingart, 2001; Pelled et al., 1999).

As a result of these two conflicting perspectives, a general disagreement exists on whether diversity in a team/group has overall positive or negative effects on performance. In his review, Bettenhausen (1991) even concluded in an overall negative effect of diversity on team performance. At the same time other researchers highlight the positive effects of diversity in bringing the group a variety of values, perspectives and behaviours and therefore enhancing the overall creativity and performance. In support of this proposition, Gibson \& Vermeulen (2003) found that diversity was positively related to team learning behaviour whereas Van der Vegt \& Bunderson (2005) suggested that team learning behaviour mediated the relationship between expertise diversity and performance. Simultaneously, some researchers predict positive as well as negative effects of diversity: Polzer, Milton, \& Swann (2002) for example revealed that high interpersonal congruence between diverse team/group members improved creative task performance while diversity undermined performance in teams/groups with low interpersonal congruence. 
Ely \& Thomas (2001) reported that according to each member's attitude toward diversity the effects on performance may be either positive or negative.

Research into the positive and negative effects of diversity on performance has moved into separate directions and an integrative theoretical framework which helps to understand both directions seems to be missing e.g. (Guzzo \& Dickson, 1996; Milliken \& Martins, 1996; Williams \& O'Reilly, 1998). Moreover, the highly inconsistent evidence for positive as well as negative effects of diversity is striking (Bowers, Pharmer, \& Salas, 2000; Webber \& Donahue, 2001; Williams \& O'Reilly, 1998). The context in which studies have been conducted has been widely neglected in explaining team/group dynamics which may be one reason for conflicting results across studies (Jackson et al., 2003). Another possible explanation could lie in the types of diversity studied and the role of intervening variables affecting teamwork and performance. Additionally, Jackson et al. (2003) revealed in an extensive review on recent research into team and organisational diversity that the majority of the studies focused on a select diversity attribute only. Studies on demographic and functional/educational dissimilarity were found to be predominant in literature (Jackson et al., 2003; Van Knippenberg, 2007). Rightly the latter argue that those studies fail to capture the full spectrum of diversity found in organisations. Van Knippenberg, deDreu, \& Homan (2004) went as far as to propose refraining from explaining the effects of diversity through typologies and dimensions of diversity. They promote giving up the strict distinction between a self categorisation perspective and an information/decision-making perspective for a more integrative view of diversity.

\section{Faultlines: Gender, Stereotypes, Language}

In a similar vain, Lau \& Murnighan (1998) suggested to go beyond the analysis of single characteristics and to investigate the interrelationship and effects of multiple characteristics to understand and to explain the dynamics and the effectiveness of teams and groups. They introduced the term 'faultlines' which refers to a division of group member's on the basis of one or more attributes. The novelty of the concept of faultlines is that groups with moderate diversity tend to experience more difficulties in establishing trust and managing conflict than groups with maximum diversity. In a highly culturally heterogeneous group/team, few common bases for categorisation, sub-group formation, selfcategorisation and social identity exist. Hence, members will try to establish a new shared understanding of processes, communication, role expectation, and the like (Earley \& Mosakowski, 2000).

Moreover, Lau \& Murnighan (1998) argue that demographic faultlines may have their strongest effect at the beginning of a team's or group's life. Yet, if team and group members do not divide along demographic faultlines at the beginning, other attributes such as gender, personality, religion or hobbies may determine the group's/team's faultline structure. For example, the emergence of 
gender faultlines may result in a division into male and female subgroups. The implementation of teams and groups in organisations has been accompanied by greater participation of females in work roles that traditionally were occupied by males (Hirschfeld, Jordan, Feild, Giles, \& Armenakis, 2006). Yet, the potential influence of gender on team and group outcomes has only been superficially researched into and stereotypes seem to prevail. For instance, females tend to be regarded to have a lower capability to succeed than males (Chattopadhyay, Tluchowska, \& George, 2004). Other stereotypes propose that sex is a more salient social category among women than men and that men are more competitive with one another than women (In: Chatman \& O'Reilly, 2004).

Research has shown that social interaction is frequently accompanied by stereotypes which directly influence perceptions. Other individuals are being categorised on the basis of easily identifiable characteristics such as race, language or age. Stereotyping also means that all individuals of the categorised group are attributed with the same roles, characteristics and skills (Husemann, 1993). This way, stereotyping is a means to reduce complexity. In fact, if stereotypes are 'right' individuals can react faster in a given situation. Moreover, they are said to be more beneficial when making comparisons between cultures than within cultures. However, they become counter productive if they do not get modified by individuals based on experience and observation in the course of team and group work (Adler, 2002)

The concepts of faultines and stereotypes are closely related to social identity theory (Tajfel \& Turner, 1986). On the one hand, individuals categorise themselves and hold multiple social identities which vary depending on context and situation (Garcia-Prieto, Bellard, \& Schneider, 2003). On the other hand, individuals categorise their team and group members into in-group and out-group members. Moreover, individuals use negative stereotypes to view out-group members as less attractive than in-group members and to maintain high levels of self esteem (Flynn, Chatman, \& Spataro, 2001).

In multicultural teams, nationality and culture often trigger faultlines and individuals use cultural stereotypes to categorise each other.

Randel (2003) proposed that culture was salient to those members of a multinational team who had the same country of origin as either few or many fellow members. Further studies on cultural diversity looked at social interactive processes in multinational groups. Vallaster (2005) revealed that effective collaboration between individuals of different nationalities occurs only when everybody understands and accepts the guiding principles or cultural values (Kirkman \& Shapiro, 2001) for their actions. Barinaga (2007) looked at two sides of the meaning of cultural difference in a multinational team. On the one hand, national culture stressed the sense of difference among group members whereas on the other hand it served to emphasize a sense of interdependency among group 
members. The 'national/cultural' discourse served both as a marker of distinction among group members and signified a sense of mutual dependency.

Another aspect of diversity and identity, namely language, seems to be forgotten in research into teams and groups. 20 years ago, Holden (Holden, 1987) remarked in a review on research into language and linguistic issues in management literature that these topics are treated with brevity by a very small number of authors only. 20 years later, Holden's remark still holds true. In fact, language studies have been treated as separate areas of research and not seen in the context of organisational studies or group and team research (Peltokorpi, 2007). This is unfortunate as numerous challenges result from language diversity: a negative impact on interpersonal relations, trust and the socialisation process have been illuminated (Lagerstroem \& Andersson, 2003). Language and communication competences become a major challenge in the socialisation process and when it comes to small talk. The interpretation of small talk in crosslanguage communications triggers more problems than the discussion of technical issues (Henderson, 2005). Hence, if language is managed effectively it enhances team building and group cohesion (Goodall \& Roberts, 2003; Holden, 2002). For instance, trust can be established by speaking the language of other team members from time to time even if it is not the main working language (Goodall \& Roberts, 2003). Nonetheless, even if all members share one common language, it is common that native speakers of one language will form closer relationships with each other, hence subgroups emerge (Chen, Geluykens, \& Choi, 2006).

Overall research into teams and groups and the effects of language still is in its infancy. We agree with Chen et al. (2006) that effective communication practises need to be identified to manage language difficulties in teams and groups. Another weakness lies in the lack of knowledge of which aspects of language impact on team and group performance and to what extent. Moreover, the impact of organisational, societal and cultural aspects on language and subsequently on performance is not yet investigated.

\section{Personality}

The role of personality is another decisive issue related to diversity. According to existing literature there is evidence that specific personality traits are supportive for team and group work (Driskell, Goodwin, Salas, \& O'Shea, 2006). Other research suggests a moderating effect of personality on e.g. acculturation of expatriates (Caligiuri, 2000).

One of the earliest investigations on the relationship between personality and team effectiveness were undertaken by Mann (1959) who found some 500 personality traits which have been measured in diverse studies conducted until that time. A reduction to the "Big Five" personality traits happened almost 40 years later when Costa \& McCrae (1992) discovered that there are about five basic but 
general dimensions that describe personality. These big five personality traits are emotional stability, extraversion, openness to experience, agreeableness, and conscientiousness which will be briefly explained.

Extroversion: the extent to which a person is outgoing versus shy. High extroverts enjoy social situations while introverts avoid them.

Emotional stability: the degree to which a person has appropriate emotional control. People with high emotional stability are self-confident and have high selfesteem, while those with low emotional stability tend toward self-doubt.

Agreeableness: the degree to which a person is friendly and approachable.

Conscientiousness: the extent to which a person is responsible, dependable, motivated and achievement orientated.

Openness to experience: the degree to which a person thinks flexibly and is receptive to new ideas. More open people tend toward creativity and action, less open people favour the status quo.

These dimensions are relatively independent and proved to hold up crossculturally. Research has also found that the 'Big Five' are related to several work behaviours e.g. to work motivation (Judge \& Ilies, 2002) and work satisfaction (Judge, Heller, \& Mount, 2002).

Many studies also refer to four respectively five dimensions established by Hofstede (2001) which are individualism/collectivism, masculinity/feminity, power distance, uncertainty avoidance, and long/short term orientation. As discussed in an interview between Hofstede \& Fink (2007) studies by Schwartz (1994) or the GLOBE study (House et al., 2004) include many more dimensions. The interview revealed that the popularity of McCrae's Big Five lies in the limited number of dimensions which are easier to relate to and also easier to remember. This is also said to be true for the four dimensions established by Myers-Briggs, namely Extraversion/Introversion, Sensing/Intuition, Thinking/Feeling, and Judging/Perceiving.

The importance of a consideration of personality traits when hiring individuals who will be placed in a team has been investigated by Morgenson, Reider \& Campion (2005). They support the notion that the big five personality traits together with social skills and team work knowledge positively influence contextual performance. Similarly, Neumann \& Wright (1999) stressed the importance of a consideration of personality traits when investigating team effectiveness. Their study proved that agreeableness and conscientiousness are predictive of effectiveness.

However, research sometimes risks neglecting the fact that the statistical significance of personality traits may be sensitive to other variables. For instance, personality traits and culture interact with each other and the question to ask is how this interaction shapes the relationship between individuals and subsequently the relationship within teams and groups. Moreover, Yukl (2002, p.203) writes: "hundreds of trait studies were conducted, but individual traits failed to correlate 
in a strong and consistent manner with leadership effectiveness". To answer how and why personality and leadership styles do (not) correlate would also be an interesting avenue for future research.

\subsection{Discussion of literature}

In the following I aim to provide general conclusions drawn from the literature review on teams and groups and specific conclusions related to the further progress of this dissertation.

\section{Clear definition of key terms}

The rather loose use of the terms "team" and "group" is the starting point of my argumentation. Researchers hardly make a reference to a definition of what makes a team or group as appendix 1 clearly shows. I conclude that it is a result of the lack of a common theoretical agreement on a definition of the terms "team" and "group". As argued earlier, the simple existence of two terms would justify a distinction. Nonetheless, it became common practice to use the terms interchangeably. Researchers may still give a different connotation to terms when they sample and even more when they interpret their findings. I argue that it does make a difference whether research is set in a self managed work team versus a team with an appointed leader or in a group of people who sometimes work together without having a common goal. The dynamics might clearly be very different. On a cultural level a clear connotation of the terms becomes even more important as different cultures have a different understanding of the concept of team/group work and associate different underlying meanings with it (Gibson \& Zellmer-Bruhn, 2001)

\section{Integration of dimensions}

A plethora of studies on demographic diversity and its effects on performance exists. At the same time, numerous studies on the influence of other factors on group processes exist. The second pillar of my argumentation corroborates that most studies look at one isolated dimension of team/group work only and that existing research into team/group performance lacks integrative research settings. As discovered earlier, most studies on the outcomes of diversity focus solely on demographical variables. Most of them don't take into consideration interactive effects with other dimensions of diversity such as functional or individual characteristics, see also Jackson et al. (2003). Out of 30 studies grouped in our category named diversity, 16 focus on demographic compositions only, five look at job related variables only, four studies involve demographic as well as job related issues, and a mere single study looks at individual aspects. That means that out of 30 studies 26 deal with pure diversity issues only. The point I wish to make is threefold: there is evidence for a tendency to conduct studies on specific variables, secondly these variables are looked at exclusively, and thirdly 
across all studies an integrative, conceptual model seems to be missing. It seems that research got stuck in dimensions and categories by conducting most studies in the same field with just slightly differing variables. Only few studies take into consideration a multi-dimension approach (Ely, 2004; Jehn et al., 1999; Kirkman \& Shapiro, 2001; Mathieu, 2006; Mendez, 2003; Randel \& Jaussi, 2003) and little pioneered on a multi-category approach (Harrison, Price, \& Bell, 1998; Jehn \& Bezrukova, 2004).

\section{Contextualisation and moderators}

As extensively discussed in 2.4.1., context is inescapable but at the same time tremendously neglected in contemporary research. Also, much is still unclear about the effects of diversity, and psychological factors etc. which are strengthened or weakened by moderators. Only recently research has started to examine the direct impact of variables of dimensions on team outcomes by also considering moderators. The set of moderator variables ranges from conflict norms (e.g.: Lovelace, Shapiro, \& Weingart, 2001), power centralisation (Bunderson, 2003), team work quality (Hoegl \& Parboteeah, 2003), to task routineness and team longevity (e.g. Pelled, Eisenhardt \& Xin, 1999). Other moderators within this scope are team cohesiveness, team interdependence, and change orientation. Thus, moderators worth considering might be the feeling for time, the role of humour, and the role of language.

\section{Qualitative research}

It appears as if research into teams/groups has come to a stage of science characterised by theory testing rather than theory building. As discussed earlier, social identity theory (Tajfel \& Turner, 1986) and self categorisation theory (Brewer, 1979) prevail in this area of research. Thus, quantitative methods dominate since they are particularly used to test theory (Creswell, 1994). Appendix 1 also clearly indicates this trend. Out of 96 studies the majority of around 70 studies followed a quantitative approach. Considering the wide range of questions still unanswered a theory building, qualitative research approach appears vital. This is for several reasons true in this specific research setting. To start off with, teamwork in the context of intergovernmental organisations is rather unexplored. Only two studies on teams and groups in the context of international organisations could be found (Elron, Halevy, Ari, \& Shamir, 2003; Neyer, 2004). However, the key word research in the journals in the field of public administration delivered no results. The six studies identified as being conducted in the public sector predominately focused on attitudinal behaviour. ArmstrongStassen, Wagar \& Cattaneo (2004) looked for example at the effects of downsizing on work-group membership while Klein, Lim, Saltz \& Mayer (2004) investigated into networks in teams. Carless \& De Paola (2000) illuminated the measurement of cohesion in work teams. Hobmann, Bordia \& Gallois (2003) 
focused on value dissimilarity, others on team member schema agreement (Rentsch \& Klimoski, 2001) or compliance and contextual performance (Van Emmerik, Lambooy, \& Sanders, 2002). Those studies clearly provide valuable insights but are far away from being integrative as argued further above.

Since the key word search on teams/groups in intergovernmental organisations did not provide a lot of results I undertook a general review on research into the field of public administration. A number of studies have compared structural characteristics of public and private organisations. Some of the most eminent researchers propose that public and private organisations are more similar than different from each other (according to Rainey \& Bozeman, 2000). Other researchers who examined for example decision making practices in public and private organisations reported notable differences (Nutt, 2005). Again, divergent opinions and research findings make it hard to bridge to the possible dynamics in teams and groups.

Consequently, the findings of the literature review bring me to the following conclusions:

- Teams and/or groups in organisations are a widely investigated field across disciplines. However, research into teams and/or groups in intergovernmental organisations hardly exists. As argued throughout this literature review, the context of a study appears to be the crucial part of it. Therefore the aim of this dissertation is to investigate whether phenomena associated with team/group work in the context of private organisations also apply to the context of an intergovernmental organisation.

- The interchangeable use of the terms "team" and "group" which might be the result of a lack of a common definition needs to be addressed again. I argue if academia is not able to find a consensus on whether or not the two terms theoretically may be seen as two different concepts of work units and as a result be labelled with a clear definition, or whether the terms may be used interchangeably, a not very well defined starting point of research will continue to lead to not completely accurately interpretable results. Even more so considering that different cultures connote different meanings to the concept of team and/or group work. I therefore let my interviewees speak and aim to provide clarification on whether or not individuals perceive to be part of a team or group. Based on the experiences and perceptions of the sample of individuals working in an intergovernmental organisation I attempt to provide a clear definition for the specific context. 
- The findings of the literature research suggest predominantly a non - integrative research approach. As argued many times before, only specific aspects of team and group work and its outcome on performance have been looked at. The aim of this study is to approach this topic in a different way by looking at three main aspects of team and group work: organisational aspects, managerial aspects and individual aspects. I argue that only an integrative view of aspects allows conclusions on the dynamics in the work units. Furthermore, the identification of moderators and the openness for asymmetric relationships will play a vital role in generating an integrative model.

As a logical consequence, an exploratory, qualitative study with clear definitions of the key terms of investigation, a rich contextual explanation and an integrative view of as many dimensions as possible seems to be appropriate and moreover necessary. 


\section{Research Methodology, Method, Research Design and Re- search Context}

\subsection{Introduction}

This chapter aims to develop the methodological framework of this research setting which focuses on team and group work in an intergovernmental organisation, and will present the research methods used. The design of the study will be presented as well as a description of the sample and the research context.

\subsection{Methodology: Grounded Theory}

Strauss and Corbin (1994, p.273) defined grounded theory as a "general methodology for developing theory that is grounded in data systematically gathered and analysed". The procedures of grounded theory are designed to develop a well integrated set of concepts that provide a thorough theoretical explanation of social phenomena under study (Corbin \& Strauss, 1990). Directly linked to this methodology is the term constant comparative method which connotes an interrelated process of data collection and analysis, an interaction between theory and data and an interplay of deduction and induction.

There are many advantages in using grounded theory. The selection of this methodology for this research setting has been directed by the following three motives:

- Grounded theory helps to develop theoretical explanations of the situations being investigated and is so close to those studied that the theory is of use to them (Turner, 1981).

- The objective of this study is to build an integrative model of how team/group work is practised in an intergovernmental organisation. Since relatively little is known about "why" and "how" teams/ groups work (or do not) in this specific context, the choice for grounded theory seems to be appropriate.

- Grounded theory as a practical method focuses on the interpretive process by analysing "the actual production of meanings and concepts used by social actors in real settings" (Gephart, 2004, p.457).

The process of theory building using grounded theory methodology can very briefly be described as follows:

Grounded theory is all about discovery and grounds a theory in reality (Glaser \& Strauss, 1967). Therefore, the research begins with an area of study and a not well defined theory. As soon as the first data within this area of study is collected, analysis begins. In the following, data collection and analysis are interre- 
lated processes. This parallel process enables the researcher to capture all potentially relevant aspects of the research topic. Each aspect discovered earns its way into the theory by repeatedly being present or by being significantly absent (Corbin \& Strauss, 1990). In the next step, data is conceptualised by comparing incidents and naming them. These concepts may then be grouped into categories by comparing, reducing and specifying them. Over time, categories can become related to one another and form a theory (Corbin \& Strauss, 1990).

\subsubsection{Method - Case Study}

Case studies are a useful research method when the research topic is well studied but a fresh perspective is needed, or when little is known of the phenomenon under study. Furthermore, a case study is a research strategy which aims to understand the dynamics within single settings (Eisenhardt, 1989). Thus, a case study can either involve single or multiple cases and numerous levels of analysis, e.g.: organisation and individual (Yin, 1984). Case studies allow for qualitative as well as quantitative data collection and analysis. Finally, case studies may be used to test theory (Pinfield, 1986) or to generate theory (Gersick, 1988). According to Eisenhardt (1989), at the beginning of a case study based on the methodology of grounded theory, it is important to start as theory free as possible. A researcher should of course identify a research area and should formulate a research question and possibly identify some potentially important variables. Yet, the researcher should avoid thinking about theories or relationships between variables as much as possible. When selecting a case, Stake (1995) describes a choice of three types of case studies: a) intrinsic cases which focus on the uniqueness of a particular case aiming to understand solely the case itself, $b$ ) instrumental cases which offer insight into a substantive issue and enable to advance theory and c) collective cases which investigate into several cases and allow a comparison between cases.

Having explained the characteristics of a case study, I believe that the study of team and group work in an intergovernmental organisation is predestined for a case study for the following reasons: Teams and groups have been extensively studied over the past decades but not in the specific context of intergovernmental organisations. To understand how individuals co-work in this specific environment justifies the application of a so called intrinsic case study. Qualitative research with embedded units of analysis will be applied to show whether existing theory in the context of multinational corporations proofs to be valid in the context of an intergovernmental organisation. Finally, a case study based on grounded theory will allow for new theory building. 


\subsubsection{Objectivity, reliability, validity, and generalisability}

Qualitative research often has been criticised as lacking in objectivity, reliability, validity, and generalisibility as opposed to hypothesis falsification used in postpositivism (Gephart, 2004). However, respecting a consistency between methodology and method and a thorough application of qualitative research methods may weaken this criticism.

To deal with the weaknesses of interpretive research, I followed the recommendations by Hussey, \& Hussey (1997), Yin (1984), Miles, \& Huberman (1994).

- Thorough explanation of methods used in study

- Respecting and following the rules of applied methods

- Documentation of all study materials (interviews, memos, assumptions and interpretations during analysis etc.)

- Triangulation by using different sources of data

- Audit of entire study

To keep as much objectivity as possible is a challenge for the researcher conducting interpretive research. Therefore, to avoid biases during the interview and during analysis, attention was paid by thoroughly following the rules of the methods applied. Reliability refers to the extent to which the results obtained could be repeated by another researcher. Validity, the core indicator in qualitative research (Bortz \& Döring, 2002), refers to the extent to which research findings accurately reflect the happenings in a specific context (Hussey \& Hussey, 1997). Validity in this research setting could be provided by respecting the five rules mentioned above. Finally, generalisibility is not a main concern of qualitative studies as this research method values the integration of a specific context and there is no primary concern about conducting the same research in completely different contexts.

Triangulation by using different sources of data (internal reports, internal case studies, books and press reports) was vital to provide reliable results. Last but not least, discussions with experts in the field (e.g. supervisors, lecturers, and staff of the UN not included in the sample) helped the researcher to cross-check conclusions.

\subsection{Research Design}

The study was designed to identify

- What kind of team and group work in intergovernmental organisations exists and 
- Factors which are a barrier, a trigger, and/or influence team and group work.

Due to the fact that relatively little is known about team and group work in intergovernmental organisations a qualitative research approach was chosen. Qualitative research aims to explain social reality by learning from the people's experiences (Flick, Karldorff, \& Steineke, 2000). Thus, qualitative research allows gathering deep insights by considering context and situational factors and as a result theory can be generated in a context in which little is known about the phenomena under investigation (Eisenhardt, 1989). Qualitative methods have been defined as procedures for "coming to terms with the meaning not the frequency" of a phenomenon by studying it in its social context (Van Maanen, 1983, p.9).

To set up this research design as a case study based on semi-structured in depth interviews has been driven by the arguments above as well as the underlying methodological assumptions.

The research design for this study comprises four iterative stages:

- field research: semi-structured interviews in 3 headquarters of an intergovernmental organisation

- desk research: literature review on teams and groups in multinational organisations

- data analysis using constant comparative method

- development of propositions and a model

\subsubsection{Semi-structured interviews}

Grounded theory research is typically associated with collecting data through interviews (Osland \& Osland, 2001). In personal interviews the interviewer is the research instrument and the interview is an opportunity to enter the mind of another and to see the world as they see it (McCracken, 1988). Consequently, this technique proves to be a valid tool to gather a broad understanding of the context the interviewees act in as well as related phenomena.

\section{Interviewing}

Grounded theory demands the researcher to enter the field of study without having any pre- existing theoretical assumptions (Glaser \& Strauss, 1967). Although I argue that it is difficult to conceive of any researcher to start a study with a theory-free mind I conducted the first five interviews without reading related literature. The first interviews were general in nature and mostly consisted of an introductory question which aimed to form a brief overview of the topic under study. According to Mayring (2002) this narrative approach allows the inter- 
viewee to openly and freely respond to the overall research question without being restricted by the interviewer. Following constant comparative method I analysed the interviews, linked them to literature and developed an interview guide for the next set of interviews. This basic list proved to be a helpful tool during interviews to make sure that all relevant topics were covered. However, a second round of interviews opened new views of the construct under investigation which I then again linked to literature and included in the interview guide. The final interview guide developed this way and has been used for the remaining sets of interviews (see appendix 2).

The interview comprised three main parts.

The first set of questions was aimed at the individual and his/her work environment. A short description of the people the interviewees work with usually led them to tell a related story. This made them feel comfortable and in the course of telling the story they naturally gave hints relating to the set of questions which was aimed at team work. Also, the interviewer could learn about the personality of the interviewee.

The second set of questions was designed to capture the role and set up of team work and to make the interviewee define what a 'team' means to him/her. An important aspect of this set of questions turned out to be the role of management that will be explained in more detail in the analysis of the data.

The third set of questions focused on the structure of the organisation and the impact of bureaucracy and red tape on the work place and team situation. Lastly, I asked the interviewees what they would wish to change in their work environment if they were in a position of power.

After the interview was over and the interviewee left, I immediately took notes on the demographics of the interviewee and the interview situation. The latter included observations made during the interview or remarks which seemed important to understand the context of the interview (see appendix 3 ).

\section{Challenges}

Applying the method of semi-structured interviews on the one hand makes sure to cover all areas of interest during the interview. The interview guide can be used to direct the conversation so that it stays on course. At the same time this technique leaves enough freedom for the interviewees to come up with unexpected descriptions and arguments. However, this technique challenges skills: the interviewer always needs to keep in mind the focus and goal of the interview. At the same time the interviewer should take advantage of the fact that most people like to talk about themselves (Bewley, 2002) and should keep the interviewee engaged and not constrained by persistent questioning. The interviewer constantly needs to find a balance between getting an answer to all questions and leaving the interviewee enough freedom to tell his/her story. Simulta- 
neously, the interviewee needs to keep track of the content of the interviewee's narratives and already formulate the next questions in her head.

Another challenge is to find interviewees who are willing to tell their story (Hermanowicz, 2002). Many people are willing to give an interview only if promised strict confidentiality (Bewley, 2002). The interviewer needs to assure confidentiality and anonymity in the introductory letter. It should engage the potential interviewees by making sure that their expert knowledge is needed and valued. During the interview it is important to create a comfortable atmosphere by asking introductory questions and by conducting small talk. As most people are busy it is important to sustain their interest throughout the interview by maintaining eye contact and also by inviting the interviewee to bring up new topics (Bewley, 2002). Furthermore, the questions need to be formulated in a way that the interviewees can understand them, and the questions need to be about topics the interviewees can meaningfully respond to.

A further topic that needs to be handled with care is the recording of interviews. Interviewees must give permission for recording. Some people might not feel comfortable and this has to be respected by the interviewer. If interviewees do not mind being recorded, it still is advisable to take notes during and after the interview because the best equipment may fail and sometimes informants may change their minds about recording (Wilkinson \& Young, 2004).

\section{Biases}

When interviewing, one always needs to keep in mind the biases which go hand in hand with this technique. In the following I would like to point out the main biases which have to be taken into consideration for this research setting, namely the cross-cultural and context bias.

\section{Cross-cultural bias}

As this study is set in a multicultural intergovernmental organisation, the researcher might be tempted to interview a variety of people from different cultural backgrounds to cover as many views as possible. However, cross cultural interviews add another dimension to the already fairly difficult technique of interviewing: culture. If the interviewer has a different cultural background as the interviewee, there is the risk that both persons attribute different meanings and interpretations to the content of the interview. This so called construct bias occurs 'if there is only partial overlap in the definitions of the construct across cultures' (Van de Vijver \& Tanzer, 1997) and it can only be avoided if both people in an interview situation belong to the same culture. Moreover, interviews across cultures are very likely to be held in languages other than the mother tongue. Either one part of the party or both parts need to express themselves in a second 
language; hence, misunderstandings and misinterpretations are very likely to happen, and as a consequence interviewer and response biases arise. Overall 'the choice and use of language as well as the researcher's and the interviewee's language skills affect the dynamics of the interview' (Marschan-Piekkari \& Reis, 2004). Furthermore, the interviewer also risks neglecting non-verbal communication. As a consequence, Fink et al., (2004) recommend refraining from conducting cross-cultural interviews to avoid cross-cultural biases unless the common third language is spoken fluently and both the researcher and interviewee are familiar with the second culture.

\section{Social desirability bias}

Social desirability can be explained as the tendency of individuals to describe themselves and the organisation they work for in a more attractive manner than they are in reality (Schwab, 1999). Especially personal or sensitive questions are prone for leading the respondent to answer socially desirable.

In this research, cooperation with other individuals and work in teams/groups can be seen as a personal issue for interviewees. Consequently, related questions may be exposed to social desirability bias. Keeping this in mind is particularly vital during analysis and interpretation of the data.

\section{Context bias}

Context as specified by Cappelli \& Sherer (1991, p.56) is 'the surroundings associated with phenomena which help to illustrate that phenomena'. As widely acknowledged and also argued throughout the literature review, qualitative research -contrarily to quantitative research - is capable of capturing contextual factors (Miles \& Huberman, 1994; Yin, 1994). Therefore, context is a vital part of qualitative research and makes models more accurate and the interpretation of results more robust (Schneider, 1985).

Marschan-Piekkari, Welch \& Tahvanainen (2004) suggest considering context at four different levels. Interview context being a potential source of bias will be explained first, followed by three other closely interwoven levels (individual context, organisational context, and external context).

The interview context as such can be described by situational variables such as the location in which the interview takes place, the time of the day, the amount of time the interviewee allows, the mood both parties are in and the interpersonal dynamics during the interview. While the location and time of the meeting can be arranged, the interviewer has no influence on interruptions during the meeting, last minute obligations on the side of the interviewee; not to mention the mood of the interviewer and interviewee the day the interview takes place. Furthermore, interviews are an arena of power relations. The interviewee might want to impose his or her meta-communicative norms on the interviewer (Yeung, 1995) which is particularly the case when interviewing elites. It is often 
assumed that the more senior the interviewer the more he or she knows (MacDonald \& Hellgren, 2004). Others argue that top management may not always know most about what is going on in the organisation and, therefore, suggest interviewing other staff in the hierarchy (eg.: Macdonald \& Hellgren, 2004). These aspects are part of the individual context of an interview referring to education, profession, hierarchical status and power of both the interviewee and the interviewer. Bewley (2002) for example reported that being a professor made interviewing very rewarding for him as interviewees paid a lot of respect to him because of the fact that he is academic. By contrast, my professor often argued that professors may represent a certain degree of authority which might intimidate some interviewees from answering the way they feel. They might fear of answering "un-academically" or they might assume that the professor is hiding his/her knowledge.

At the second level, the type of organisation under study (its size, structure, strategy, culture, and history) forms the organisational context of the interview (Marschan-Piekkari et al., 2004). A thorough consideration and description of the organisational context is suggested (Rousseau \& Fried, 2001). The latter ones argue that organisational factors are very often taken for granted by the interviewer who fails to consider organisational influences during the interview. The same may also apply to interviewees who may personalise events and exclude organisational effects. Contrary to the organisational context, which is also known as inner context, the external (or outer) context encompasses the national culture, the political and economic environment in which organisations are embedded (Pettigrew, 1985). A constant consideration of the external context during analysis is recommended in order to avoid false assumptions.

To avoid biases, a rich description and consideration of context on all four levels during the interview as well as during analysis is highly recommended. This is even more so in qualitative research which demands a rich description of context.

\subsubsection{Data Analysis - Constant Comparative Method}

With the permission of interviewees, interviews were recorded, transcribed and linked to personal characteristics: demographic ones such as age, gender, education and functional characteristics such as position, experience.

The text material was fed into software Atlas.ti, Version 5, which helps to code, match and administer qualitative research data. It allows to quantify the findings and to draw network views. The software does assist in data analysis such as data reduction, categorisation and administration, but the actual analysis, interpretation and theory building is in the hands of the user of the software. 


\section{Constant Comparative Method}

As described in the seminal work of Glaser \& Strauss (1967) four stages of constant comparative method have been respected throughout the data analysis:

1. Comparing incidents applicable to each category

2. Integrating categories and their properties

3. Delimiting the theory

4. Writing the theory

Step 1: Comparing incidents applicable to each category

The beginning of analysis using constant comparative method is dominated by the discovery and creation of codes through interpretation of data. As explained by Hussey, \& Hussey (1997) codes serve to enable the data to be segmented, compiled and organised. Strauss \& Corbin (1990) talk about three different kinds of codes: open codes, axial codes and selective codes. Open codes are the basic form of codes which come from the researcher's own imagination or from literature (vivo codes). In the course of open coding, the researcher assigns names to contexts, events, activities or stories found in the data. The analytic process of open coding leads to an identification of concepts which are the basis for the development of theory. In a next step, through axial coding, the researcher tries to make connections between categories. Once again this is an iterative process and the researcher goes back and forth between open and axial coding. In a last step, selective coding results in the development of a core code which is the central phenomenon that has emerged from axial coding.

The coding process involves both inductive and deductive thinking. Inductive thinking means that categories, themes and patterns emerge from the data material itself whereas deductive thinking verifies existing theory and frameworks against the data (Fink et al., 2004; Patton, 2002). Furthermore, coding and categorising shows a constant interplay between proposing and checking as explained by Glaser \& Strauss (1967, p. 106): 'While coding an incident for a category, compare it with the previous incidents in the same and different groups coded in the same category'

A constant comparison leads the researcher to generate theoretical properties: the researcher may eventually relate categories to each other and start to think in terms of the full range of types of categories, their dimensions and their major consequences.

After coding for a category for possibly several times, Glaser and Strauss (1967, p. 107) suggest stopping to code and, instead, to recording a memo of the researcher's own ideas. 'The analyst should take as much time as necessary to reflect and carry his thinking to its most logical (grounded in the data, not speculative) conclusion.' Furthermore, it is advised to write memos throughout the process of analysis to illustrate the researcher's ideas. 
Step 2: Integrating categories and their properties

As the coding continues, constant comparison causes categories to become related to other categories and as a result accumulated knowledge is built up. As different categories become integrated, the researcher can start to develop theory (Glaser \& Strauss, 1967, p. 109).

Step 3: Delimiting the theory

This step is mostly characterised by reduction, which comprises bundling categories to a smaller set of higher level concepts. Consequently, reduction leads to a more precise terminology. Thus, the researcher complies with two requirements of theory: parsimony of variables and their formulation and scope in the applicability of the theory to a wide range of situations (Glaser \& Strauss, 1979, p. 111).

Step 4: Writing theory

After having carefully respected the first three stages, the researcher finally arrives at writing a theory based on the categories developed, their relation to each other and their integration to a whole concept. The content behind the categories is provided by the memos which represent the major themes of the theory (Glaser \& Strauss, 1979, p. 113).

\subsubsection{Sample}

As this study deals with team and group work and the conditions under which this type of work is enhanced (or not), I decided to conduct interviews at a macro-level unit of analysis. Hence, 50 semi-structured, face to face and individual interviews in three locations of five Organisations of the United Nations were conducted between June 2005 and June 2006. To avoid a cross-cultural bias, the decision was made to only interview Austrian and German employees. Contacts were made through networking and 50 interviews could be conducted. All interviews were conducted by the author and lasted between 45 minutes to 2 hours. The interviews mostly took place in the cafes of each of the three locations visited. With permission, interviews were recorded and taped. Total anonymity was promised in every case.

The demographics and functional characteristics among the people interviewed are very diverse and the sample represents a wide variety of staff members.

Table 3 below summarises the characteristics of the interviewee sample: 
Table 3: Demographics of the sample

\begin{tabular}{|l|l|}
\hline Demographics of sample & Frequency \\
\hline Age & \\
\hline $20-30$ & 8 \\
\hline $31-40$ & 20 \\
\hline $41-50$ & 14 \\
\hline Over 50 & 8 \\
\hline Gender & \\
\hline Male & 30 \\
\hline Female & 20 \\
\hline Education & \\
\hline University degree & 37 \\
\hline No university degree & 13 \\
\hline No. of languages spoken & \\
\hline 2 & 20 \\
\hline 3 & 23 \\
\hline 4 & 7 \\
\hline Time of tenure & \\
\hline 1-5 years & 23 \\
\hline 5 -10 years & 12 \\
\hline Over 10 years & 15 \\
\hline Position & \\
\hline Head of Department/Director & 5 \\
\hline Professional Staff & 18 \\
\hline Junior Professional Officer & 8 \\
\hline General Staff & 14 \\
\hline Intern & 5 \\
\hline Organisational unit & \\
\hline IAEA & 18 \\
\hline UNIDO & $9 *$ \\
\hline UNDP & 8 \\
\hline UNOPS & 5 \\
\hline WIPO & 5 \\
\hline Others & 5 \\
\hline & \\
\hline
\end{tabular}

*one person has been interviewed twice (before and after leaving the UN)

\subsection{Research Context}

The interviews took place in three locations of the United Nations, namely in Vienna, Geneva and New York. At a lower organisational level, five organisational units were chosen: the International Atomic Energy Agency (IAEA) in Vienna, the United Nations Development Programme (UNDP) in New York, the United Nations Industrial Development Organisation (UNIDO) in Vienna, the United Nations Office for Project Services (UNOPS) in New York, and the 
World Intellectual Property Organisation (WIPO) in Geneva. The reason for this choice was twofold: first, the huge body of principle organs of the United Nations System with its numerous related agencies, organisations and programmes cannot possibly be investigated by a single researcher within the limitations of a single research project. However, to get a broader understanding of the structure and culture of the United Nations Organisation it seemed necessary to conduct interviews in different types of units. Therefore, I aimed to conduct interviews in agencies, programmes and organisations with diverse functions, diverse structures, and diverse cultures and last but not least, diverse locations.

Second, within these units a sufficiently large number of German speaking staff who were willing to give interviews could be identified.

The following details aim to describe briefly the history of the United Nations Organisation and its structure as well as the diverse units under investigation.

\section{Context}

'The United Nations Organisation (UN) is an international organisation bringing together 191 states which have committed themselves voluntarily to a mutual obligation to safeguard peace and humane living conditions for the people of the world' (Gareis \& Varwick, 2005).

In June 1945, in the aftermath of World War II, 50 founding members gave birth to the United Nations by signing the Charter of the United Nations in San Francisco. Over the years, a further 140 states have become members of this intergovernmental organisation.

The United Nations System consists of six primary organs which will briefly be explained in the following (source: (Gareis \& Varwick, 2005):

- The General Assembly: intergovernmental forum for consultation and cooperation. All member states are represented equally by one vote per state.

- The Security Council: responsible for world peace and international security. 5 permanent and 10 non-permanent member states; meets on a daily basis.

- The Economic and Social Council: works together with General Assembly on economic and social topics; 54 members.

- The International Court of Justice: primary juridical organ of the UN, consist of 15 independent judges appointed by Security Council and General Assembly.

- The Secretariat: main administrative organ. The Secretary-General is elected by the General Assembly on the recommendation of the Security Council and serves for 5 years.

- Note: The Trusteeship Council: suspended its work in 1994 after the transition of the last trustee 
Apart from these 5 main organs, the United Nations System also consists of various, sometimes independent, decentralised organisations and programmes, each with its own by-laws, membership, structure and budget (Gareis \& Varwick, 2005).

\section{International Atomic Energy Agency (IAEA)}

The first United Nations Organisation to be located in Vienna was the IAEA in 1957. The IAEA counts as an autonomous, international organisation within the United Nations System and the two bodies consult each other. A special agreement between the UN and the IAEA governs their relationship with regards to reporting, exchange of information, cooperation, technical assistance, budgetary and financial agreements and personnel arrangements. The IAEA is responsible for international activities concerned with the peaceful uses of atomic energy. It finances itself out of regular budget resources and voluntary contributions. The IAEA reports to the Security Council and the General Assembly. (Source: [www.iaea.org] downloaded 15.05.2007)

\section{United Nations Development Programme (UNDP)}

The UNDP emerged in 1965 as an independent instrument for the planning, financing and coordination of development projects. It specifically administers the technical part of these projects which aim to fight poverty and to improve living conditions. The funds for the projects are based on grants which do not have to be repaid by the developing countries. The UNDP has no formal membership. It is financed through the voluntary contributions of member states. The central office is in New York but it has offices in more than 130 countries and is involved in some 5.000 projects at any given time (Gareis \& Varwick, 2005). UNDP reports directly to the General Assembly and has a non-subsidiary relationship with the Economic and Social Council.

\section{United Nations Industrial Development Organisation (UNIDO)}

UNIDO was established in 1966 as an organ of the General Assembly. Like the IAEA, UNIDO functions as an autonomous organisation within the United Nations. Its permanent headquarter is in Vienna. UNIDO is represented in 50 developing countries (thereof predominantly in Africa). The two main areas of activities are to strengthen industrial capacities and to encourage cleaner and sustainable industrial development.

Funding for UNIDO activities is drawn from the regular budget, the operational budget, and voluntary contributions. (Source: [www.unido.org], downloaded 15.05.2007) 


\section{United Nations Office for Project Services (UNOPS)}

UNOPS was established as a self-financing unit at the beginning of 1995 and provides operational management services such as reduction of red tape, loan administration and supervision, but also protection of international waters and biodiversity or electoral assistance. The clients of UNOPS are United Nations System partners such as UNDP, UNICEF, UNESCO and country governments. UNOPS is self-financing and charges fees on estimates of the actual costs of rendering services to a client. UNOPS reports directly to the General Assembly. (Source: [www.unops.org], downloaded 15.05.2007)

\section{World Intellectual Property Organisation (WIPO)}

In 1967 WIPO was established by the WIPO Convention to promote the protection of Intellectual Property. The headquarters is located in Geneva. WIPO is a specialised agency of the United Nations dedicated to developing an accessible international intellectual property system among its member states. This includes development of international laws and treaties regarding patents, trademarks, and industrial designs, copyright and related issues. WIPO administers fee-based services based on international agreements which enable users in member countries to file international applications for patents and international registrations for trademarks, designs, and appellations of origin. WIPO is largely self-financing with 90 percent of the budget coming from earning from provided services. (Source: [www.wipo.int], downloaded 15.05.2007)

Table 4 summarises the main characteristics of the organisational units described. It shows that these five units differ in many ways: the choice of units covers a United Nations programme (UNDP), an office (UNOPS), an agency (IAEA), and two organisations (WIPO, UNIDO). The units not only differ by the status of the entities under investigation within the United Nations System but also in the way they are financed (self financing and/or regular budget), with regards to their main area of work (internal and/or external) and their function (technical and/or services). Last but not least, they are also located in different headquarters. 
Table 4: Unit summary

\begin{tabular}{|c|c|c|c|c|}
\hline Unit & Reports to & Financed by & Function & $\begin{array}{l}\text { Loca- } \\
\text { tion }\end{array}$ \\
\hline IAEA & $\begin{array}{l}\text { Security } \\
\text { Council, } \\
\text { General } \\
\text { Assembly }\end{array}$ & $\begin{array}{l}\text { Self-financing } \\
\text { because } \\
\text { autonomous } \\
\text { agency }\end{array}$ & $\begin{array}{l}\text { Peaceful use of Atomic } \\
\text { Energy } \\
\text { technical }\end{array}$ & Vienna \\
\hline UNDP & $\begin{array}{l}\text { General } \\
\text { Assembly }\end{array}$ & $\begin{array}{l}\text { Voluntary con- } \\
\text { tributions }\end{array}$ & $\begin{array}{l}\text { Administers technical part } \\
\text { of development pro- } \\
\text { grammes }\end{array}$ & $\begin{array}{l}\text { New } \\
\text { York }\end{array}$ \\
\hline UNIDO & $\begin{array}{l}\text { General } \\
\text { Assembly }\end{array}$ & $\begin{array}{l}\text { UN budget, } \\
\text { operational } \\
\text { budget, mainly } \\
\text { voluntary con- } \\
\text { tributions }\end{array}$ & $\begin{array}{l}\text { Public and private sector } \\
\text { services. Technical: de- } \\
\text { sign and implementation } \\
\text { of programmes }\end{array}$ & Vienna \\
\hline UNOPS & $\begin{array}{l}\text { General } \\
\text { Assembly }\end{array}$ & Self-financing & $\begin{array}{l}\text { Mainly UN internal ser- } \\
\text { vices }\end{array}$ & $\begin{array}{l}\text { New } \\
\text { York }\end{array}$ \\
\hline WIPO & $\begin{array}{l}\text { General } \\
\text { Assembly }\end{array}$ & $\begin{array}{l}\text { Self financing, } \\
\text { charging of } \\
\text { fees for ser- } \\
\text { vices }\end{array}$ & $\begin{array}{l}\text { Development of laws; reg- } \\
\text { istration of patents and } \\
\text { trade marks (services) }\end{array}$ & Geneva \\
\hline
\end{tabular}

Source: author 


\section{Empirical Findings - Data Analysis}

\subsection{Introduction}

This chapter forms the vantage point for the development of theory on teams and groups in an intergovernmental organisation. The analysis procedure of 50 qualitative, semi-structured interviews followed the grounded theory and constant comparative method approach as formulated in the methodology and methods part.

As argued throughout the previous chapters, at the heart of qualitative research and data analysis is a rich description. Therefore, emphasis will be put on the explanation of the development of patterns and their implications.

This chapter will start with answering the question whether or not group/team work exists in the investigated Organisations of the United Nations. The aim is to provide a definition for the constructs revealed during this research. Furthermore, the factors which enhance or hinder team/group work in this specific context will be explored and described. Moreover, it will be tested whether phenomena discovered in a non-governmental environment are also valid in an intergovernmental environment.

Finally, results of the findings of the analysis will be aggregated into a conceptual model.

\subsection{Team or group work? A typology grounded in the data}

The first research question 'Do teams/groups in the United Nations exist and if so, what are they like?' forms the point of departure of this research. The stories told by 50 individuals working in five different Organisations in the United Nations were analysed in detail to clarify which kinds of team and group work are practised.

The explanation of how team and group work is perceived in five United Nations Organisations will be developed in a threefold manner. Firstly, those characteristics will be explained that the majority of all interviewees relate to team and group work. Single quotes that best capture the content of these characteristics will be used as illustrations. Secondly, this study reverses the direction of discussion by evaluating characteristics of team and group work which were reported to be missing. Thirdly, the characteristics reported will be compared with theoretical definitions of team and group work. The analysis concludes in a typology of constructs. 
Figure 2: Towards a typology

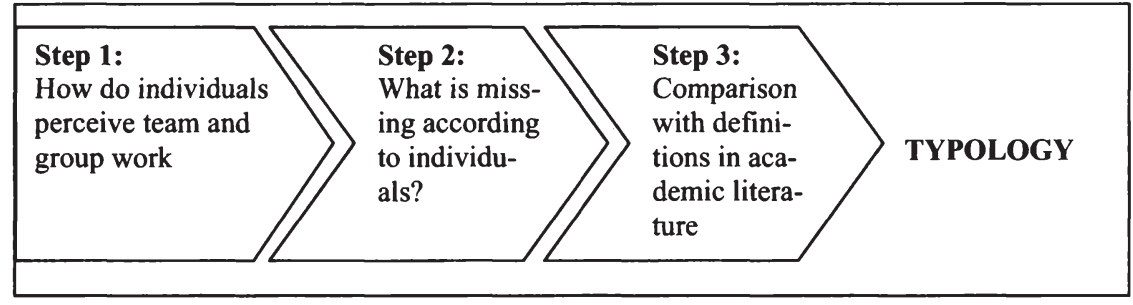

Source: author

Step 1: How do individuals perceive team and group work

The first step of data analysis revealed that the basic question "Do you work in a team or group" could not be answered straight forward. The most common approach interviewees chose to answer the question was to describe the hierarchical position of both co-workers and supervisors and to list the different nationalities interviewees work with. The following quote is a typical sample for a response:

Well, I had a supervisor from Sweden and then from Belgium. [...] And my colleague from Haiti has been here for a while as well. (P9:06)

Another pattern which emerged in this first step of analysis is that interviewees tried to position themselves as a specific type of member of a team or group. The narratives would start with ' $I$ am ...' and result in a self-categorisation of interviewees as either a member or a leader of a team or group.

I work with 5 technical officers in a group and in collaboration with another officer. (P20:19).

I am a group leader. (P26:17)

I am a team leader of 5 persons. (P35:11)

I am part of a planning team in a project. (P41:13)

$90 \%$ of interviewees then described their tasks and responsibilities. They were of a very different nature depending on the Organisation the interviewees 
worked for and depending on the type of staff the interviewees belong to. However, and regardless of the function of interviewees, two patterns emerged. On the one hand, interviewees highlighted the importance of management as the main factor of whether or not team and group work functions. Interviewees referred to the responsibility of a team and group leader to develop processes within the team and group to make it work. These processes are among others the communication style, the extent to which information is shared among staff, the coordination of tasks or the way how decisions are made. The quote below refers to how the likehood of conflicts has been reduced by setting up specific processes:

I have learned to direct certain team processes right from the beginning before team work degenerates into chaos and conflict. (P19:28)

The narratives around management were numerous and extensive and will be explained in more detail in the course of the data analysis.

On the other hand, an element of team and group work alludes to personality of individuals as a factor of whether or not team and group work is construed, and how it is accomplished. All interviewees reported at some stage that the personality of co-workers was a crucial element in group functioning. Personality was explained to be a stronger factor of influence than nationality and hierarchy. Personality too will be explained in more detail in the further course of this analysis.

The first step of data analysis further showed that around $60 \%$ of all interviewees interpretations of team and group work can be categorised around two intertwined behaviours which interviewees relate to team and group work: communication and coordination. According to the majority of the interviewees, communication involves the exchange of information and knowledge among the members of a team and group.

In my view, collaboration in teams stands for transfer of thoughts and knowledge. (P17:22)

A distribution of information provides each member with the information that is necessary to fulfil a task. The interviewee below accentuates equal treatment of all members in a network:

... equality for all, everyone gets information on anything and participates. Whether or not they are able to participate is another question but there is no separation of information. No. there is a network in which everyone is equal. When we plan a project all of our colleagues 
work on it, everyone adds a part and everyone corrects the others... (P29:26)

Moreover, the exchange of information is described as being intensive in order to gain as much background as possible:

It is a process, which consists of a lot of exchange with the person that stands between XX and the American who does the analysis. Also, there is a lot of exchange because this person has been working here for a long time and knows a great deal. He is Austrian. And I personally, try to understand the background of everything and to develop myself because I have not been working here for a long time. This means for me constant questioning why things are the way they are. $[\ldots]$ the rest is operational work which I do all by myself. (P11:120)

The means of communication may vary from email to written reports or meetings (reported by $90 \%$ of interviewees).

The extent to which team and group members share information among themselves depends on the coordination of the team and group with regard to tasks and resources:

Each person is allocated specific tasks. For example a press conference is being organised - I need to inform the media, I have good contacts with Austrian and international media. Interviews need to be organised and the TV stations as well. Each person always has the same task and knows what to do. (P21:08)

As described by the majority of interviewees, coordination is executed in a top down approach. The example below derives from a high ranking employee who is responsible for the strategy and development of a project:

Well, I have another responsibility now in a coordinators role, called human security. [...] on one hand, I develop strategies, strategies for the house, what does UNIDO do in this area, which services do we offer, what impact do our programmes have and on the other hand it is project development. And here we work together with technical departments and this means teamwork. Teamwork on another level; meaning that we develop a project together. (29:08) 
Step 2: What is missing according to individuals?

When I asked interviewees 'How do you define team or group work?' they became aware of the difference between the two concepts. However, despite this awareness, the terms team and group are words that were used very loosely: the majority of interviewees predominately used the term team, a considerable number mainly referred to the term group but most of the time interviewees used the terms interchangeably in the course of their narratives. Even when explaining the concepts it became clear that the simple use of terms does not mean that the concept beyond it corresponds to the terminology used. Individual work does not become group work simply because it is called so nor does group work become team work for the very same reason (Katzenbach \& Smith, 1993).

At the same time, some interviewees realised that - according to their very own definitions of team and group work - some major characteristics might be missing in their current work situation to make it a team.

One of these elements reported by interviewees is performance measurement. Around $25 \%$ of interviewees reported either a lack of performance measures or the wrong means of measurement:

They [management] should look at what they [group/team] achieved and set indicators to measure their performance. (P18:12)

There are performance indicators for projects but they have relatively little implications. (P19:93)

Of course there are performance evaluation reports for the individual. One can say that one has developed this and that but that does not say anything about the success of the project whatsoever. This is definitely missing. (P29:35)

Around $10 \%$ reported a lack of participation in the decision making process:

They (the supervisors) say that we can participate in the decision making process but in the end only for non-essential issues. (P40:20)

Another $10 \%$ of interviewees remarked that the overall structure of the United Nations Organisation is based on a hierarchical model and therefore does not allow team work.

Consequently, a summary of the first two steps of data analysis on how individuals construe team and group work delivers the following insights. Narratives on team and group work are in $\mathbf{4 5}$ out of 50 interviews associated with organisa- 
tional characteristics such as hierarchy and bureaucracy. The work situation is described by referring to the organisational context which comprises a diverse workforce with diverse national and cultural origin. Almost $90 \%$ of interviewees explain team and group work by relating it to hierarchical positions and functions of individuals in those positions.

Despite the fact that interviewees were not provided with a definition for the terms 'team' and 'group' work but rather had to try to define the constructs themselves, more than half of all interviewees acknowledged that some elements which may characterise real team and group work might be missing in their daily work lives.

Step 3: Comparison with theoretical constructs of team and group work in academic literature

In the next step of analysis I compared the explanations of the interviewees with regard to team and group work with theoretical concepts in academic literature. This step comprised a comparison of definitions of the terms 'team' and 'group' to determine which type of work - group and/or team work - exists in the United Nations Organisations under investigation.

A clear definition for the underlying concepts needed to be provided, hence, the simple but comprehensive definition by Katzenbach \& Smith (1993) seemed appropriate for this research setting.

Definition of the term "team":

A team is a number of 2 or more people who are committed to a common goal which is specific and different from each team member's individual goals. Team members work collectively to reach this goal. They share leadership roles and follow a collective decision making process. Furthermore, the solution to problems is found in the course of collective discussion. The outcomes of the team work are subject to collective performance measurement.

Definition of the term "group":

A group is a number of 2 or more people who are committed to a common goal which is similar to the broader organisational goals and each group member's individual goal. Group members work individually to reach their goals. The leadership role is centralised at one person who is also responsible for the decision making process and who delegates individual tasks. Finally, the outcome of group work is subject to individual performance measurement.

Table 5 summarises the main differences between a team and a group: 
Table 5: Team vs. group requirements

\begin{tabular}{|l|l|}
\hline Team & Group \\
\hline $\begin{array}{l}\text { Specific goal which is exclusively de- } \\
\text { livered by the team }\end{array}$ & $\begin{array}{l}\text { Goal is the same as the broader or- } \\
\text { ganisational mission }\end{array}$ \\
\hline Shared leadership roles & One strong leader \\
\hline Collective decision making & Centralised decision making \\
\hline Collective work-products & Individual work-products \\
\hline $\begin{array}{l}\text { Collective discussion and solution of } \\
\text { problems }\end{array}$ & Delegation of individual tasks \\
\hline Collective performance measurement & Individual performance measurement \\
\hline
\end{tabular}

Source: adapted from Katzenbach \& Smith (1993)

This clear distinction between the terms team and group allowed for a typology of what kind of team and group work exists in the United Nations Organisations under investigation.

A comparison of the interview data with the requirements for team and group work showed that the individuals' work environment is strongly influenced by hierarchy and bureaucracy. As a result, highly ranked staff within the hierarchy is in a position of power, makes decisions and delegates tasks. Task specialisation permits individual performance measurement. Thus, formal requirements for team work are not met. The data however refer to the existence of group work.

Based on the different definitions and underlying explanations of interviewees, three typologies of group work could be established and are grounded in the data. In the following, task forces, project groups, and collaboration and their categorisation into a typology of group work will be explained in some detail and illustrated by sample quotes.

\section{Task forces}

When the organisation needs something special to be done, it creates a small group to do it. This small group is brought together temporarily for a specific purpose and exists only until the problem is solved. Management literature calls these task forces 'temporary groups comprising people with complementary 
skills who work to a common purpose for which they are collectively accountable' (Child, 2005, p.102).

Task forces are flexible. They can focus on whatever problem of immediate importance. They often bring together people from different sections or divisions of the organisation to work on a cross-functional purpose. In this way, task forces use information and knowledge from several sources which may induce new ideas and generate new directions. Rules and regulations may sometimes be simplified for task forces. There is a dedicated leader of the task force. Decision making is based on hierarchical position. All of these characteristics are grounded in the interview data and illustrated by sample quotes. Task forces are explained to be set up for two specific purposes: non-routine purposes and emergency events.

Interview 31: Task force for non-routine purposes

There were ad hoc groups, hundreds of them. These ad hoc teams could be found in the whole UN. High ranking officials were responsible for the important issues. They gathered for non-routine-purposes which - in my view - were much more interesting. (P31:26-28)

There were also special teams for annual meetings. The special advisor had a supporting team as there was tremendous administrative work which had to be done under extreme time pressure while certain formal standards had to be maintained. A well functioning team was therefore very important. (P31:38)

Interview 42: Task forces for emergency events

It works like this: there is a catastrophe. Let's take the Tsunami as an example. We then needed many people, 50 or so, and once again, we break it down to 5 people, 5 people are a team which works somewhere in coordination. [...] and then we select the team here. Our office is responsible for this and one of us here [...] leads this team most of the time. We - senior people - lead the teams. (P42:24)

Our administration just called. They work for us in a simplified way [...] we do not have to go through the same administrative system like the other agencies. This is emergency response and all our deadlines and rules for recruiting and sending off people into the field and applications for money are shortened. [...] recruitment can be done within 48 hours. (P42:48) 


\section{Project groups}

A project group according to the interviewees is established for the duration of a specific project. There are certain rules how to apply for a project, how to develop project work plans, and about the duration of projects. Furthermore, reports about the progress of the project have to be delivered on a regular basis. Once the project is completed, group members are reassigned to new projects or their contract is determined. If the goal could not be reached the project may be extended. These project groups have a designated project leader. Tasks are distributed according to specialisation.

Project groups are reported to be found in technical units like the IAEA as well as in UNDP. The following quotes illustrate the findings:

Interview 20: Project group in IAEA

The application for the current project idea started in Sep. 2005. We are currently evaluating the applications, and at the end of 2006 we will visit the country and prepare a detailed plan of work for the next 2 years. The project will start in 2007 and run for 2 years. After 2 years you decide to extend the project or not. It depends on whether they reached their goals or not. But there will be reports every 6 months to see how the project is going. (P20:19)

For example we need to talk to farmers. I don't actually do it myself, the scientists there are responsible for it. But we bring a certain technology there and hope that it is successful. (P20:11)

I work with 5 technical officers in a group and in collaboration with another officer. (P20:15)

\section{Interview 13: Project groups in UNDP}

The work in small groups is running. There are 120 persons and they work in groups of 5 to 10 persons, there are project manager, decision makers and supporting staff, another 5 persons who do the paperwork etc. Every 6 weeks there is a huge meeting which is more like an informative management meeting. (P13:77) 


\section{Collaboration}

Wagner (1995) defined collaboration as a 'wilful contribution of personal effort to the completion of interdependent jobs'.

This form of work was reported by interviewees within all five Organisations under investigation. Typically, this form of collaboration involves communication and exchange of information among individuals. However, there is not necessarily a common goal collaborating individuals work for. Their collaboration might be a result of work in the same division or under the same manager.

Interview 4: Collaboration and Management

Everyone is involved, everyone has to describe his/her job. $\mathrm{XY}$ is the quality manager who supervises everything. (P4:45)

Interview 23: Collaboration and Co-Workers

For technical requests an Austrian technician is responsible, he is my contact person and I work very closely with him. (P23:04)

Interview 38: Collaboration and task

It is repetitive, a lot of routine, the process and the workflow is very well defined. (P38:16)

The typology presented shows that the extent to which group work takes place strongly depends on two factors: the timeframe and the degree of task specialisation. Task forces operate on non-routine tasks and are set up at an ad-hoc basis for a short period of time. Project groups work on well defined tasks for a longer period of time. Collaboration implies high task specialisation and unlimited timeframe. 
Figure 3: Types of group work

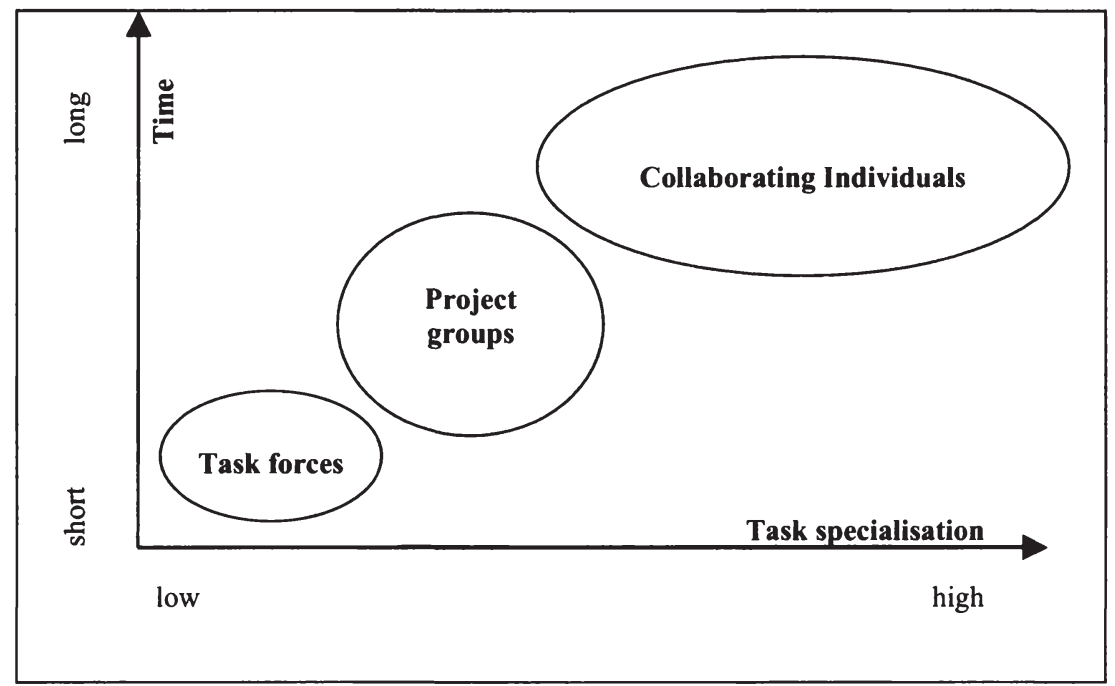

Source: data analysis

In a follow up conversation with five selected interviewees from different hierarchical positions and organisations I presented the typology of groups that evolved from the first part of data analysis. This feedback loop was very important to make sure that the narratives of interviewees and my interpretations of them do not digress. The typology was approved by the control group of interviewees. After interviewees were provided with a clear definition for the term team and the term group as shown in table 2, all of the five interviewees acknowledged the difference between the two concepts. Moreover they agreed that team work by this definition does not exist in the five United Nations Organisations under investigation due to structural restrictions of the overall organisation, which will be explained in the next part of analysis. Table 3 illustrates the typology of group work which emerged in the course of data analysis and by relating the data back to theory. The table gives an overview of all findings: 
Table 6: Definition of groups

\begin{tabular}{|c|c|c|c|}
\hline $\begin{array}{l}\text { Type of } \\
\text { group }\end{array}$ & Definition & Most significant quote & Task \\
\hline $\begin{array}{l}\text { Task } \\
\text { Forces }\end{array}$ & $\begin{array}{l}\text { For non-routine events or } \\
\text { emergency events. Highly } \\
\text { knowledgeable and/or es- } \\
\text { pecially skilled staff is as- } \\
\text { signed fully to an event. } \\
\text { E.g peacekeeping teams or } \\
\text { high-level staff teams }\end{array}$ & $\begin{array}{l}\text { It works like this: there is } \\
\text { a catastrophe. Let's take } \\
\text { the Tsunami as an exam- } \\
\text { ple. We then needed } \\
\text { many people, } 50 \text { persons } \\
\text { and once again, we break } \\
\text { it down to } 5 \text { persons, } 5 \\
\text { persons are a team which } \\
\text { works somewhere in co- } \\
\text { ordination. [...] and then } \\
\text { we select the team here. } \\
\text { Our office is responsible } \\
\text { for this and one of us here } \\
\text { [...] leads this team most } \\
\text { of the time. We - senior } \\
\text { people - lead the teams. } \\
\text { (P42:24) }\end{array}$ & $\begin{array}{l}\text { Trained } \\
\text { emer- } \\
\text { gency } \\
\text { tasks e.g. } \\
\text { earth- } \\
\text { quake or } \\
\text { military } \\
\text { mission. } \\
\text { Highly } \\
\text { intellec- } \\
\text { tual tasks } \\
\text { and/or po- } \\
\text { litical de- } \\
\text { cisions }\end{array}$ \\
\hline $\begin{array}{l}\text { Project } \\
\text { groups }\end{array}$ & $\begin{array}{l}\text { Established to fulfil a } \\
\text { specified task or project } \\
\text { within a certain time } \\
\text { frame. On completion } \\
\text { members are reassigned to } \\
\text { new projects or end con- } \\
\text { tract (modification based } \\
\text { on Child, 2005, p.105) }\end{array}$ & $\begin{array}{l}\text { The work in small groups } \\
\text { is running. There are } 120 \\
\text { persons and they work in } \\
\text { groups of } 5 \text { to } 10 \text { persons, } \\
\text { there are project manager, } \\
\text { decision makers and sup- } \\
\text { porting staff, another } 5 \\
\text { persons who do the pa- } \\
\text { perwork etc. Every } 6 \\
\text { weeks there is a huge } \\
\text { meeting which is more } \\
\text { like an informative man- } \\
\text { agement meeting. } \\
\text { (P13:77) }\end{array}$ & $\begin{array}{l}\text { Clearly } \\
\text { specified } \\
\text { in project } \\
\text { outlines, } \\
\text { interre- } \\
\text { lated tasks }\end{array}$ \\
\hline $\begin{array}{l}\text { Col- } \\
\text { labora- } \\
\text { tion }\end{array}$ & $\begin{array}{l}\text { Wilful contribution of per- } \\
\text { sonal effort to the comple- } \\
\text { tion of jobs (based on } \\
\text { Wagner, 1995, p.152) }\end{array}$ & $\begin{array}{l}\text { For technical requests an } \\
\text { Austrian technician is re- } \\
\text { sponsible, he is my con- } \\
\text { tact person and I work } \\
\text { with him very close. } \\
(\text { P23:04) }\end{array}$ & $\begin{array}{l}\text { Routine } \\
\text { tasks, well } \\
\text { defined, } \\
\text { individual } \\
\text { goals }\end{array}$ \\
\hline
\end{tabular}




\subsection{Properties of group work}

The first major step of data analysis revealed that group work summarised in three broad typologies is practised in the five United Nations Organisations under investigation. In a further step the properties of group work will be explored. All properties are derived from the interview data. Some of them emerged in the first step of analysis towards a typology; others emerged in the course of iteration and constant comparison.

The properties can be grouped around three main categories which emerged in the course of analysis, the first being the organisational level of the overall United Nations System comprising the Organisation's structure and processes. Second, the group level incorporates employee-management relationships and employee-co-worker relationships, and third, the individual level comprising personal attributes of individual employees represents another property of group work. Moreover, boundary spanning activities were identified to act as a moderator.

Figure 4 shows the process of how the categories emerged and led to generate a conceptual model. Each category which represents a property of group work will be explained in more detail in the remainder of this chapter which will conclude with the presentation of the conceptual model. Propositions will be deduced from the empirical analysis and formulated at the end of this chapter.

Figure 4: Emergence of categories

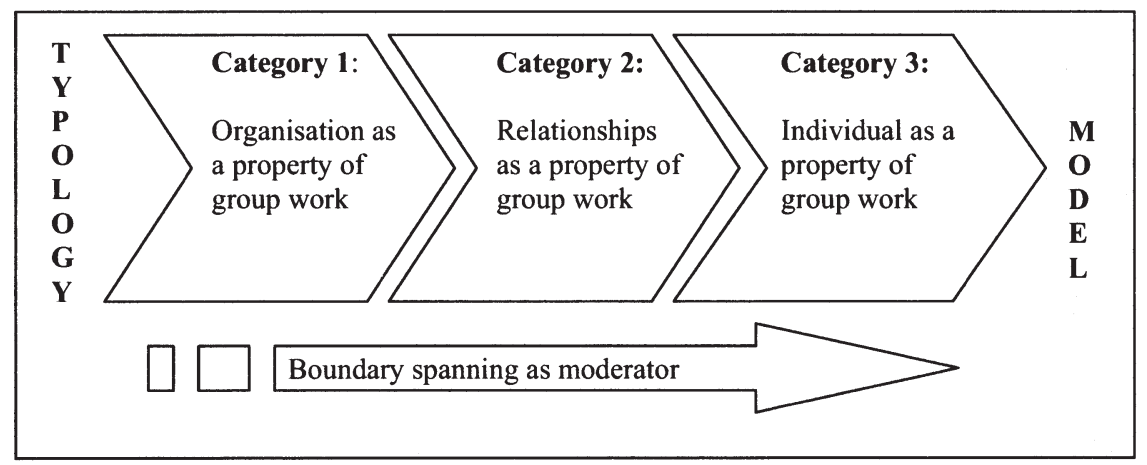

Source: Data analysis 


\subsubsection{Organisation}

\subsubsection{Structure of the organisation}

Like all organisations, the United Nations Organisations have a structure, lines of authority, formal communications, formal policies and formal rules (Beigbeder, 1987). In other words, the structure of the organisation is determined by hierarchy and bureaucracy.

According to Child (2005), hierarchy, as part of the structure of an organisation, is depicted by different positions of power within the hierarchy and adjunctive authority as well as reporting lines. The specialisation of tasks takes place according to functions like units, groups and roles. Furthermore, the structure of an organisation contains procedures to clarify to staff what is expected from them. This is translated into rules and standards (bureaucracy).

\subsection{Hierarchy}

Hierarchy is a fact of both human and organisational life. The bigger an organisation the more hierarchically structured it usually becomes. This way, organisational members can distinguish between different levels of responsibility, authority and power. It also identifies accountability.

In a conventional organisation, hierarchy is characterised as follows (adapted from Child (2005, p.61):

- Positions are structured into clearly differentiated levels

- People at higher levels have greater authority and responsibility and take decisions that are riskier and more complex

- People at a higher level have the authority to give instructions or request to those lower and are ultimately responsible for the people at lower levels

- People at lower levels have the right to ask for guidance or instructions from the ones above them

- Leadership is exercised top down through command, coordination and control

- People at the higher level receive information before it is passed down to the lower level

A purposeful way to explain the hierarchy of the United Nations Organisation is by using the United Nations Systems' salary structure which mirrors the hierarchical structure of the entire organisation.

The United Nations salary system comprises 15 ranges of salary. The number of ranges is dependent on the number of hierarchical levels in the Organisation. The skills which are required for a job, the responsibilities of the employee, the supervisor or subordinate status of staff are determined by the level they occupy in the structure. 
The majority of the affiliated Organisations of the United Nations System are divided into 15 ranges. Only recently, select Organisations were consolidated into four main ranges or so called bands (Fernandez, 2005) as shown in the figure below:

Figure 5: Hierarchy of Staff in UN

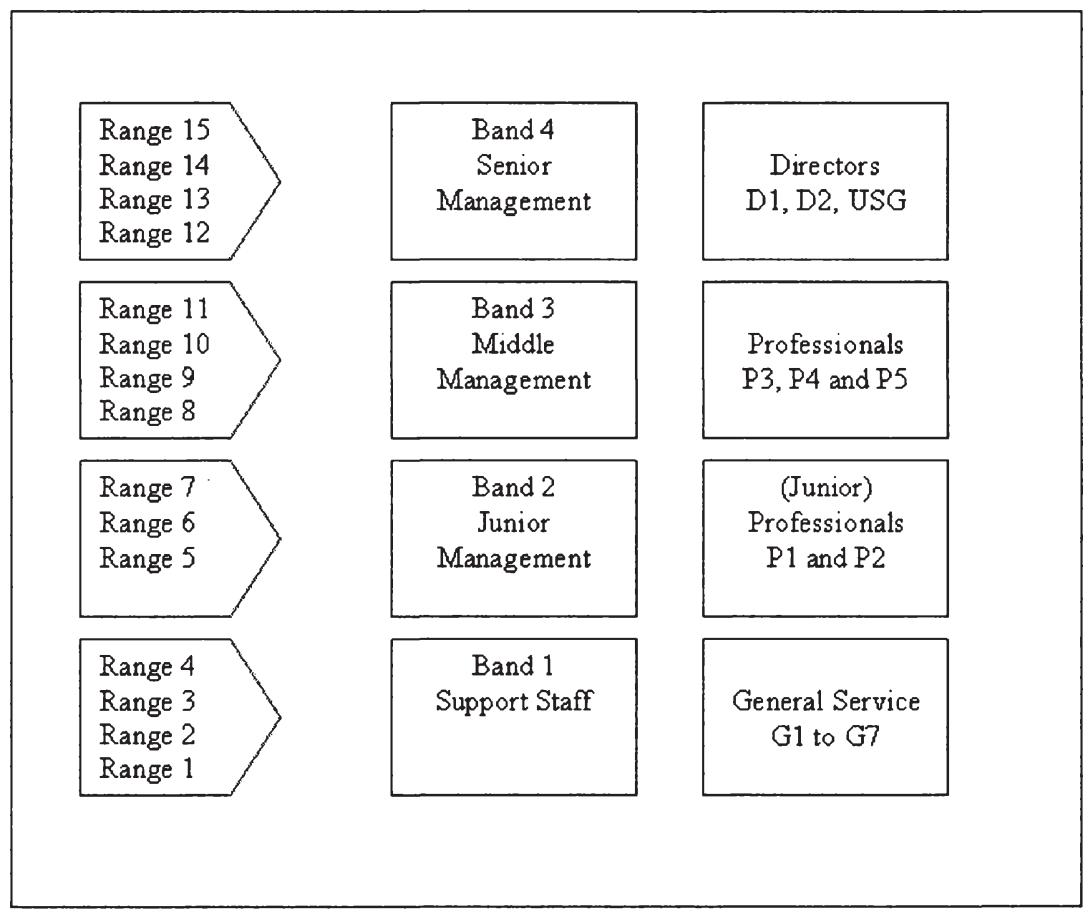

Source: modified from Fernandez, 2005, p. 149

Band 4 staff (or D-staff) is divided into two Director Levels (D1 and D2) as well as the levels of Assistant Secretary-General and Under-Secretary-General in some organisations and Assistant Director-General and Deputy Director-General in others. D-staff represents the highest level of staff within the hierarchy. Job duties involve, for example, strategic and / or political responsibilities. Band 3 staff (P-staff) or professional staff comprises three grades: P3, P4, P5 whereas band 4 comprises two grades, namely P1 and P2 (includes also JPO, Junior Pro- 
fessional Officer). These middle and junior management positions involve management activities and are quite frequently based on contracts with a specific date of expiry and therefore have to be renewed from term to term. Thus, the principle of rotation applies to all of the bands described so far. Furthermore, staff on the professional and director level usually are recruited internationally and are paid on the basis of salary scales which were established by the General Assembly and apply worldwide.

Finally, band 4 (G-staff) in the General Service comprises seven levels from G1 to G7. General Service staff is recruited locally and broadly speaking is responsible for administrative tasks. G-staff traditionally holds permanent contracts which are not subject to renewal. Overall, each band is connected to specific tasks, responsibilities and job related specialties such as the rotation principle.

Both the traditional as well as the broadbanded structure show that there is a strong hierarchy within the United Nations Organisations System. The existence of a strong hierarchy is also emphasised by the individual perceptions of $90 \%$ of all interviewees:

[...] what strongly characterises the $\mathrm{UN}$ is that there are strong hierarchies. Well, it is all ... work is in line. Yes. The hierarchies are very strong. (P30:12)

The hierarchy is very strict, I personally find that this is not beneficial for the outcome...let's put it this way. That does not mean that I really want to complain about it but it is a fact that it is very hierarchical here...yes, there is a section head, from an actual head to director general and back...so it is all very hierarchical. (P39:39)

A point which might need to be considered which is negative is this strong hierarchical system...that everything works according to fixed rules...it is very narrow and one does not have a lot of freedom, almost any step is given somewhere and has to be respected ...but whether this is good or bad is not up for discussion. (P2:22)

\subsection{Bureaucracy}

As touched upon briefly above, hierarchy often arises hand in hand with bureaucracy expressed by rules and regulations. The idea of bureaucracy is generally traced to Woodrow Wilson's (Wilson, 1887) influential article 'The study of administration' and Max Weber's work (Weber, 1922, 1962, 1978). To Weber, bureaucracy was a system of administration carried out on a continuous basis by trained professionals according to prescribed rules (Beetham, 1996). 
The key features of the ideal type of bureaucracy according to Weber are (Allison, 1984):

1. Official functions organised on a continuous basis and regulated through a system of rules and procedures

2. A division of labour so that each function has its specific sphere of competence

3. A hierarchical structure providing for the supervision of lower offices by higher ones

4. Technical rules and norms - their application requires that officials have received appropriate training, thus possess technical qualifications

5. A separation of the property of the organisation from that belonging to the official as a private individual

6. A written record of all administrative acts, decisions and rules

Bureaucracy is a means to describe relationships between organisational members in two ways: on the one hand as a relationship of power and on the other hand as a relationship of care (Sewell \& Barker, 2006). When bureaucracy works through the operations of hierarchy and rule based conduct, it forms a relationship of power. Consequently, bureaucracy can be seen as an instrument of power and control dominated by the top level of a hierarchy.

Complementary to this, bureaucracy may also be seen as a means to protect the organisation and its individuals from adverse actions of managers or co-workers. This ambiguity is also reflected in the interview data:

... in the UN many bureaucratic steps are undertaken because we need to control each other... [...] the bureaucracy in the $\mathrm{UN}$ is based on mutual distrust, which, yes, this is sometimes important to say because control is sometimes better than trust. (P13:39)

Well there are many many things one needs to know and which might be considered as bureaucracy in the broadest sense. But I think that this is indispensable. If an organisation is a certain size, this organisation needs a framework, a structure, which can be controlled and which people can work within. Unfortunately it does not work otherwise. (P29:48)

The bureaucracy of the United Nations System is a rather old bureaucracy in comparison with other intergovernmental organisations like, for example, the European Union. Thus, the United Nations System has experienced a lot of re- 
forms since the foundation of the system in 1945. These reforms where due to the dramatic operational expansion of the United Nations Systems throughout the years (Annan, 2006).

In the course of reforms, several internal reports have repeatedly reported one major structural problem of the United Nations System as expressed in the following:

- UN rules and regulations are too complicated and too numerous to serve as clear guidance for staff (Beigbeder, 1997)

Despite numerous attempts to reform the bureaucratic system, complexity is still a fact of organisational life ( $90 \%$ of interviews).

One interviewee argued that because of the long existence of the bureaucracy and the various changes due to reforms and changing general directors, the overall bureaucracy became even more complex throughout the years:

The UN bureaucracy has no logic. It has grown but the ones who make the bureaucracy only stay for 2 years and their successors do not look at what the people previously have done and so it all becomes quite illogical. (P13:81)

Or as stated by another interviewee:

It (the bureaucracy) is terrible! Each general director promises to reduce it but this never happens...it is absurd, there is practically no control in the web and you need 1000 signatures for even the smallest things. (P23:14)

Another staff member who worked for the United Nations 20 years ago, left to become self-employed and has returned to work on a 3-year contract remarked the following:

Well I have the feeling that it all slowed down. Purchasing for example used to be much faster in the past, now one needs much more time.

Recently it has taken 4 month to order equipment. (P24:16)

There are too many subdivisions. Each huge organisation structures a lot, too much, the processes become very long. (P24:16)

In general, the interviewed staff perceived bureaucracy to be extremely strong and especially hard to learn when beginning to work at the United Nations Or- 
ganisation. This point has been emphasised by the majority of interviewees who also mentioned the need to rely on the help of others to get acquainted with bureaucracy:

$\mathrm{Oh}$, it is a disaster. The processes and protocols are amazing. It takes 400 times longer than learning the alphabet. But you know, they have to be accountable and there are so many projects that's why they need all the protocols. So basically, I think it is all because of accountability. But the processes themselves are not clear. I am glad I have people I can ask. (P22:12)

Another interviewee described a feeling of being lost in bureaucracy by referring to being an apprentice who has to learn from scratch:

I have the feeling, well, I feel like an apprentice again. (P41:92)

The feeling of being lost in rules and regulations was also shared by another interviewee who has spent several years in the United Nations. The interviewee distinguishes between 'official rules and regulations' and 'personal rules' set up by individuals and not by the system. As touched upon earlier, the bureaucracy of the United Nations is a rather old one and has repeatedly changed, but also seems that it has been gradually built up over the years. These procedures are outlined in the following quote which helps to give a feeling for what awkward really means:

Up to now I still don't know how and why things work and others don't. There are different levels. On one hand there are relatively many rules, general rules and specific UNOPS rules which demand a lot of bureaucratic processes. And then there is the next level [...] I'd like to give an example: there are UN rules which state how to do the purchasing. If we have finally signed a contract, then, according to the rules, the producer has to send the original invoice. Why the original invoice...this originates from the old times ... supposing there was just one invoice which could guarantee that a) the invoice is correct and b) it is not paid twice. We still carry along the processes of the old system; we say that we need an original invoice. Well, we receive the original invoice; the project manager receives it, that's only a personal thing that does not have anything to do with rules. The project manager copies the invoice and passes it on to his payment assistant; the payment assistant copies it for personal records and passes it on the finance. Finance holds the original. This means that paper load is 
tripled but this is not a rule, it simply is like that because one day someone started doing it like this. If there is no original invoice because it came by fax it has to be approved by the division chief which does not make sense at all because a division has around 700 projects. And to know of 700 projects which one is an original invoice and has someone twiddled something does not make sense. [...] Anyhow, the invoice has to be signed and makes its way through many hierarchical levels. It takes a long time until it finally gets paid. Our task with this business process reengineering is to find out where do rules apply and where do we find processes which have developed over the years and became a habit. (P44:75)

However, the remarks about bureaucracy by the various interviewees should also be put into perspective. It is a kind of stereotype that public or intergovernmental organisations have particularly high levels of rules and red tape, hence bureaucracy. A number of studies have compared structural characteristics of private companies and public or intergovernmental organisations (Rainey \& Bozeman, 2000). Opposing the existing stereotype, these studies did not report significantly different levels of red tape (Bozemann \& Loveless, 1987). This view was also shared by a few interviewees who have had working experience outside of the United Nations System:

...to be honest, in huge companies like Bertelsman or IBM or others it was the same, it has to be like that. You need the different checks and balances. (P16:13)

If something becomes big, all huge organisations tend to document everything, to streamline it and then you have all these procedures which one has to respect. Everything that becomes big becomes relatively inflexible, on the other hand it becomes manageable because of the process... (P9:44)

Hmm...the bigger the company the more bureaucratic it becomes. It was the same when I worked at Ericsson, people had enough to do without producing any output. They were so occupied internally that no one would notice that there is no output. (P2:93)

Regardless of whether differences or similarities were highlighted throughout various studies, it was found that any activity related to personnel administration typically faced much more red tape in government organisations (Bozemann, 
2000). This topic will be investigated in more detail in the following section as it was a significant factor in the interview data.

\subsubsection{Human resource management}

A general objective of Human Resource Management is to find staff who match the abilities and attitudes desired by the organisation to provide just compensation and benefits and to guarantee continuous career management (Fernandez, 2005).

Being an international, intergovernmental organisation with 191 member states challenges the Human Resource Management of the United Nations System as each member state shall be represented proportionally in the various United Nations Organisations and gender equality shall be provided (UN, Charter). The subsequent implications are structured in the following into topics such as recruiting, contracts, career development and termination of contracts.

\section{Recruiting}

The United Nations System has strict recruitment policies to ensure transparency, equality and fairness. These policies are reflected in very detailed job descriptions. Furthermore, the venue and length of advertisements is clearly specified and the selection of personnel is subject to strict rules. Overall, the recruitment procedures are indeed complex and lengthy. On average it takes 174 days from the time a vacancy is announced to the time the candidate is selected (Annan, 2006).

This was also pointed out by two interviewees:

It can take months until one gets an answer. I applied for a job in May and it was September when I was informed that I would have an interview. (P21:28)

HR is very slow, everything has to work according to specific rules...but I think this does not depend on the people but on the processes. (21:32)

A speciality of the recruiting system is that the United Nations Organisations generally look to promote existing staff before recruiting from the outside by, for example, reassigning staff from headquarters to perform various field jobs and project related work (Fernandez, 2005). This is possible because of the diverse workforce that exists within the whole United Nations System which is one of the advantages of intergovernmental organisations. One interviewee high- 
lighted the advantages of this recruitment process and described the internal application process as well as the possibilities to climb the hierarchy as follows:

There is a tender which means that all posts are part of the tender. If I find an interesting job on our website I can apply for it, then I might be invited to an interview and maybe I get the job. This means I can always apply for something else. For example, my job is a G-4 position. If I have 5,6,7 years of work experience, that's most of the time the minimum, and maybe my section head retires, then I will apply for his job, which is a G-5 job. (P9:76)

However, one of the downsides of staffing in intergovernmental organisations results from political appointments. According to two interviewees:

This is a political organisation. So there are 191 member states, they are governments; they are not people, so they have their own interests. [...]. But then sometimes you know, member states lobby certain posts, high level posts, it is all a fact of life. So you have to take that into account. It is not only about performance. But that's a fact of life in the UN. (P47:122)

Sure, the environment is very political here. But somehow it is just like in the public authorities... with a new government comes a new minister... (P39:70)

\section{Different contracts}

Another downside of the United Nations Human Resource Management is evident in the plethora of different types of contractual arrangements. On the one hand, they are difficult to administer as the contracts range from permanent to monthly contracts with enormous variations between and among contracts. On the other hand they create inequality and resentment between staff as it is a fact of life that staff work side by side in similar positions but receive different financial remunerations and services.

An interviewee makes the point:

When it comes to contracts there is extreme variety. There are people who handle themselves from one 3-month-contract to another and there are people who hold permanent contracts, quasi for the rest of their lives. And in between there are various nuances with regards to the length of a contract. There are people who do similar jobs but have very different contracts. (P44:39) 
Yet another issue which arises as a result of differing lengths of contracts is reflected in the rotation of higher ranking staff which is due to rotation policy. The latter involves rotation of usually higher level staff between positions and locations on a regular basis (between 1-3 years). Interviewees explain that this rotation policy triggers constantly changing procedures:

I see a problem in the enormously strong change. The frequency is very likely one and a half years for international staff. Very often international staff is the high ranking staff which makes the decisions. And that's where I see the big problem ...because of a lot of rotation and changes in decisions. It's just like if you work in an Austrian company and the persons who make decisions change every year, and then there are also problems. (P13:48)

The rotation policy is seen twofold: on the one hand a regular change of high ranking staff leads to consistently changing decisions and practices and hinders stability while on the other hand it seems to be indispensable when it comes to political issues. Furthermore, rotation does not only have negative aspects but also positive ones if, for example, it ensures continuous learning and progress. One interviewee highlighted the differences between the need for stability and the need for progress:

P-Staff have this rotation policy so they are in a continuous war because people want to keep their job. The personal interest is first priority. This is a problem but at the same time the rotation policy is necessary for 2 reasons

There should not be the same people all the time. New blood/new ideas bring evolution. You need to be always at the top of the technology and therefore you need new people with new ideas and that's why it is fair. [...] And they should choose people on real competency. But of course there is political pressure from member states; they want to have their people in place. It is difficult to have a really competent base and a political system. (P18:09)

This struggle is also reflected in the following quote:

The big problem of the UN is the fluctuation that people leave because of the rotation policy. At the same time the system stabilises other and carries them along even though you would not need them any more. (P13:79) 


\section{Career development}

The types of contract individuals hold directly influence their possibilities for career development. Furthermore, career development is obviously limited by education, skills and hierarchical position. Like in any other organisation, promotions may only be granted if the employee possesses the necessary qualifications. However, in the United Nations there are two more dimensions which influence career development: geographical origin and the existence of plan positions within the hierarchy. The staff interviewed perceive the issue of geographical posts as follows:

Permanent staff at the UN that's geographical posts, you really need to come from an underrepresented country and you need to fulfill so many requirements to receive the job, it is not really your knowledde. (P43:44)

Some positions aren't an option if you are the wrong nationality. But you have to live with it, it is the same in politics. (P29:64)

However, on the other hand, staff have a very realistic view of personal possibilities within the career path based on education and skills and the existence of plan positions:

A promotion, to get the job of my supervisor, is practically impossible because of my education and the fact that I am Austrian. I won't make the next step. That's not possible because for the job you need to be academic...only if you are the only qualified candidate then you get the P-post even though you are Austrian and under qualified. (P8:65)

I have limited career possibilities. Let's put it this way...they are limited because a lot of planned posts have been cut. If there are no planned posts I can do five times the job of someone else who has a higher ranked post. If there are no posts there are no posts. And that's the disadvantage of international organisations because if you work in an international company you boss can promote you more easily. That's not possible here. (P9:62).

However, some staff feel disadvantaged in the promotion process and point this back to the management:

The management often does not place staff in suitable positions. People are not asked, suddenly there is a new org chart and some people 
are shifted. One the other hand some people do not get shifted at all. (P27:33)

\section{Termination of contracts}

The termination of contracts, especially permanent contracts, is extremely difficult and tedious. At the same time contracts of politically appointed staff can hardly be terminated because of their political nature. As a consequence, people stay within the United Nations even though their performance is unsatisfactory. The following comments best capture these insights:

You have to something really bad to be dismissed. It happens but very rarely. (P24:24)

That's an absolutely huge problem. I don't think it is possible to fire people unless you do something really really horrible and it ends up in the newspaper. It's very difficult to get fired in the UN. (P47:110)

In the end these people are put into unimportant positions and are carried along by the system. (P13:75)

The issues covered above have been raised in different internal reports of the United Nations over and over again during the course of the past decades. As a result, several reforms have been introduced to solve these problems. One of these reforms introduced in 2002 aimed at promoting staff on merit and competence and less on tenure and precedent (Fernandez, 2005). The outcome, according to Fernandez (2005), has been a streamlining of procedures which reduced the selection and recruitment process to 90 days.

In conclusion, I would like to refer back to the beginning of this chapter where I described the strong hierarchy of the United Nations Organisation with its strict categorisation of staff into different bands. As already explained and illustrated by quotes, this hierarchy restricts the individuals' possibilities for skill development because certain training is only available for certain types of staff. Moreover, it limits career development as promotions are still based on seniority and time in the position and not on performance or merit. This is also true for the remuneration of staff as the current pay system is not sensitive to performance but solely based on position within the hierarchy. This system triggers implications on motivation, competition, knowledge sharing and individual behaviour, which will be discussed in the following section. 


\subsubsection{Moderator: Boundary crossing and boundary spanning}

Another component of an organisation, according to Child (2005), is the boundary-crossing component which is traditionally concerned with drawing lines of exclusion between units and jobs by a clear specification of responsibilities and authority. It also involves networking as an alternative to hierarchies depicted by communication across an organisation's boundaries. The latter is also known as boundary spanning. Generally speaking, boundary activities as described by Cross, Yan \& Louis $(2000$, p. 843$)$ are those 'in which an organisational entity engages to create and maintain its boundaries and to manage interactions across those boundaries'. This organisational entity may be an organisational unit, a group or team or an individual.

Boundary spanning in literature is closely linked to bureaucracy and hierarchy and aims to reduce the amount of red tape as well as the number of levels in the hierarchy. One way to achieve this might be by implementing cross-functional or team-based structures (Denison, 1996).

On an individual level, boundary spanning may be related to networking, communication and exchange of information. Individual boundary spanning arises if communication across organisational boundaries is inefficient, time consuming or too costly (Katz \& Kahn, 1966).

In the following I will first explain the triggers for boundary spanning in this specific context. Furthermore, I will illustrate individual boundary spanning activities by quotes before concluding with boundary activities enacted by the organisation.

\section{Triggers for boundary spanning}

Coming back to the initially cited report which depicted the problems of the United Nations System, the list established therein continues as follows (Beigbeder, 1997):

- There is a lack of both horizontal and vertical communication

- Institutional memory needs improvement

These two points address the double-edge sword characteristics of hierarchy which have already been referred to in the previous section. Although hierarchies are said to be better mechanisms to transfer knowledge than, for example, markets (Kogut \& Zander, 1993), data analysis showed that this is not perceived in the five Organisations under investigation. On the one hand, hierarchy assures clear lines of report, responsibility and control. On the other hand, hierarchy restricts managerial efforts, career and skill development. Consequently, hierarchy limits information exchange, alas communication, and as a result, it limits knowledge transfer. Consequently, institutional memory can hardly be built up. 
Knowledge as defined by Davenport \& Klahr (1998) is 'information combined with experience, context, interpretation and reflection'. Literature distinguishes between two types of knowledge: explicit and tacit knowledge. Explicit knowledge can be coded and stored by writing it down while tacit knowledge is mainly in people's heads and more difficult to capture and to make explicit (Polanyi, 1967).

This is reflected in the interview data as illustrated by the following quote:

It is much more bureaucratic than I had thought, much more hierarchical and each department cooks its own soup and the departments know relatively little about what is going on in other departments. And what I notice is that this organization lacks knowledge management. It simply does not exist here. For example if you search a specific document you are lost here. And the processes duplicate, I think this is because the departments do not communicate with each other. There is also no knowledge pool, no information pool to get information and knowledge from. (P41:37)

Another interviewee explains the lack of knowledge transfer to expectations related to different jobs, suggesting that in a certain position specific skills and specific knowledge are expected, and therefore there is little need to share or transfer knowledge:

We were the first ones in this office. It did not exist before. This means that everything was very confusing, there was nothing and I was expected to have the ability to manage this position and to work according to the terms of reference. Strickly speaking, one does not need training or knowledge transfer.

Interviewer: And your successor also did not receive any training?

No, I don't even think that I will get to know him/her. I know the application process, it will take a long time until my successor will be here. I only have to finish my work so my successor does not have to work on it any more and I need to put all the data in templates on the PC. It will all be available on the computer and he/she will have to work with it. I only leave access codes and make a written handover. (P10:11) 
Another interviewee points to the lack of communication and information sharing across organisational units:

What I have noticed is that there is rather competition than cooperation between the UN agencies. there are tasks which partly overlap, each department names it differently but in reality it is the same task. However, they all need donors. So the donors can decide who has got the better proposal and that's the reason why the internal cooperation is not so strong. I for example was looking for a paper from UNDP and wanted to borrow it from the library. But they said that it is a paper and that I have to pay for it. Well, there is competition especially when it comes to funding of projects. It is always about money and each signature is probably about money as well. (P12:48)

Furthermore, to continuously ensure accountability, hierarchy is supported by bureaucracy with its rules and regulations. Power is given to those who can change the rules and regulations (D and $\mathrm{P}$ staff) but also to others who know how to apply and execute these rules and regulations (G staff).

As a result, exchange of information and communication becomes part of a game of power. In the worst case, knowledge is not shared. An interviewee makes the point:

G Staff is extremely important because it is not replaced. The people know why there are rules and how they work. Without G staff the organization would not work. G staff are a living memory, we leave after a couple of years but they stay. Motivated G-staff are extremely positive but at the moment when they become negative it becomes a hurdle per se. If they (G-staff) don't help you, you make one mistake after the other. (P24:33).

\subsection{Individual boundary spanning}

To reduce their information deficit, and to work beyond hierarchical limitations, individuals start to span boundaries. Research has for example shown that external contacts are used to obtain information, support or resources (Ancona \& Caldwell, 1988). Other research suggests that leaders can be the impetus for boundary spanning (Cross et al., 2000).

Overall, there are many ways to span and cross boundaries and each individual might follow a different approach and a different strategy. Some start to communicate outside the hierarchical lines of communication, others start to network and others build friendships and favour each other. The following quotes 
are examples of different ways of individual boundary crossing and spanning in the United Nations Organisations under investigation:

It sounds funny but I just walk around and ask all the people and somehow I get the information i need. I always try to find it somewhere but I have to say that it is hard to find info on the intranet which we now have [...] I have realised that emails and telephone calls mostly are not so effective which means I just walk up onto the $5^{\text {th }}$ floor and say I need this and that and then the people are always friendly and I get information provided they have the knowledge. (P41:41)

In my case it is easy, I know the people, I know where to go and what to do. (P21:27)

I have a great uni network and many connections. For example, you need an import licence for a device from the US. Still the are not allowed to send it to us so they send it to me at uni and we also have got it. If you want you can bypass anything. You can only make it through the networks the individuals have, the UN does not have it. The UN does not have the money to build up networks and resources that's why the UN takes on the right people. The UN buys P-staff to fulfil specific tasks. (P24:26)

There are key people who are the link from one team to another. The informal network is much stronger than the formal network. It is much stronger than the hierarchy. (P42:50)

There is a discrepancy between hierarchy and its formal usage. There is a parallel system. (P39:44)

\subsection{Organisational boundary spanning}

In the organisational context of the United Nations System, the strongest indicator of boundary activities is manifested in various organisational reform processes. The Secretary of the United Nations articulated in various reports that the organisational structure needs to be reformed and to address new needs and requirements demanded of an organisation in the $21^{\text {st }}$ century. New governance principles, a strong management, and horizontal coordination over vertical hierarchy are the primacies.

Yan and Louis (1999) see the main organisational forces to span or even bring up boundaries in de-bureaucratisation, the use of teams, a shrink of organisa- 
tional slack, increased workforce diversity and advanced information technology.

The following quote illustrates activities in place in the United Nations System referring to de-bureaucratisation:

I have an internal project...business process reeingineering. We look at processes and how to make them faster, more effective and more accountable. Some processes need a lot of time and energy without showing any effect so we try to open up the process at some point to make it faster. In the $\mathrm{UN}$ a lot of paper is being moved and we try to cut it and to introduce more intelligent systems. The problem often is that there are papers with seven signatures. This takes a lot of time and energy without having any effect so we try to implement faster procedures. (P44:43)

\subsubsection{Culture of the organisation}

A plethora of definitions of the culture of an organisation exist. In a most general description, the term refers to shared meaning suggesting that the culture of an organisation is carried in the values and behavioural norms of organisational members. Moreover, culture is a process and a product of people within an organisation. 'People interacting in an organisation create their culture through their interactions with one another, culture is produced not for them but by them' (Gortner, Nichols, \& Ball, 2007, p.156)

A longtime researcher of organisational culture, Edgar Schein (1984), has proposed the following definition: 'Organisational culture is the pattern of basic assumptions that a given group has invented, discovered or developed in learning to cope with its problems of external adaptation and internal integration, and that have worked well enough to be considered valid, and, therefore, to be taught to new members as the new way to perceive, think and feel in relation to those problems.'

Despite definitions, organisational culture is a concept which is hard to capture and the question is how to assess culture.

Research suggests that culture exists on several levels. Schein's influential model (Schein, 1985) suggests that that there are three levels of culture: the first level comprises observable manifestations of culture. These artefacts are for instance symbols, the physical arrangement of work spaces or the pattern of communications and how power is expressed. The second level comprises the values and behavioural norms which underlie these artefacts, and at the third level lay beliefs and assumptions. The latter ones are the core essence of culture according to Schein. 
Figure 6 shows a slightly amended version of Schein's model of culture which comprises all three levels of culture.

Figure 6: Schein's model of culture

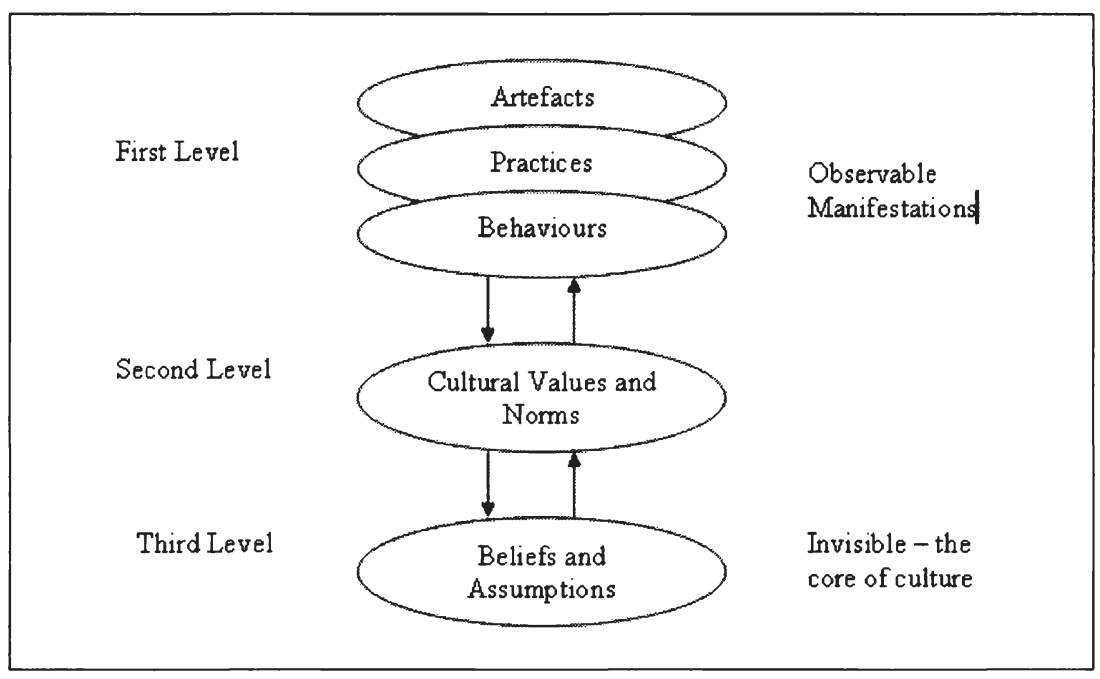

Source: adapted from Schein (1985)

In a similar vein, Hofstede (2001) for example summarises manifestations of culture in an onion diagram which comprises three layers, namely symbols, heroes, and rituals. At the core of the onion lie values. The three layers are visible to an outside observer while values are invisible and only become evident in behaviour.

This first level of organisational culture was represented in the interview data as quoted in the following:

The size of the office and the number of windows tell you quite a lot about the status of the person. (P39:79)

There is a difference between staff and delegates in this house...there are two discussion-cultures. (P1:17) 
Moreover, culture becomes visible in the jargon and terminology organisational members use. This aspect comes into it's own in the quotes of interviewees. All of them used at one stage of the interview expressions of a second language as a result of the mixed use of different working languages, especially English and German. Most of the time sentences were a mix of both languages as demonstrated in the following sample which is a quote by an Austrian staff member. Out of the 36 words of this single, supposedly German sentence, 10 are English:

In fact this is true for all international employees and the WIPO is a specialized agency of the UN so we are all part of the UN family, we have the same staff regulations and rules and management principles. (P40:47)

Another indicator for organisational culture is that it provides an intrinsic sense of belonging for the individual. Instrumental theory (Jiménez, Górinak, Kosic, Kiss, \& Kandulla, 2004) argues that identification and the feeling of belonging are induced by perceived personal interests. In the case of the United Nations this is reflected in the belief of individuals that their belonging to this organisation and their work has a sense of meaning and impact. This is also called the symbolic-interpretive approach to culture (Geertz, 1973). According to Weick (2001) making meaning is also an issue of organisational culture. As expressed by interviewees:

I do work with a feeling that I have greater influence. The projects apply for so many countries and it is great responsibility because it gives help to people all around the world. (P22:25)

On this international level one has the feeling of being able to exert influence. (P14:69)

The second level of organisational culture is composed of the deeply held values, beliefs, assumptions, and attitudes that underlie behaviour. This level of culture is not as visible as the previously described level of organisational culture. However, it can be made visible when looking at shared meanings of individuals who work in a specific organisation. Key characteristics of shared meaning are amongst others communication patterns which influence the relationship between individuals and their managers as well as individuals and their coworkers. Hence, individual values and beliefs can be unfolded by looking at different types of relationships. This level of culture will be explained in the following section which focuses on the relationship between employees and management and employees and co-workers, and the individual who is the agent in these relationships. 


\subsubsection{Employee - Management relationship}

\subsubsection{Management - the crucial link}

The former General Secretary, Kofi Annan (2006), strongly emphasised in the millennium summit the improvement of Human Resource Management (HRM). The weakness of the HRM system specifically lies in the neglect of:

- the build up and development of competent management; and

- the importance of participation of staff members to develop and maintain their skills and competencies through constant learning and development.

Being aware of managerial problems within the system, Kofi Annan initiated in 2005 a study which aimed at improving the quality of selection and training of heads of the United Nations funds and programmes. This study was carried out by the Office of Internal Oversight Services and an external consultant. The outcome, presented in a multipart report in June 2006, is a comprehensive review of governance and oversight within the United Nations, funds, programmes and specialised agencies (OIOS, 2006). This report is especially interesting for this dissertation as it covers (amongst other units) all the units in which interviews for this research were conducted. Furthermore, one part of the data derives from 160 interviews with managers and addresses several management issues which will be mentioned in the further analysis.

The report highlighted the importance of a better training of managers which allows managers to reflect on their management style, to learn recent management techniques as well as to apply them. These measures aim to develop future managers who possess the necessary leadership skills as well as cross cultural and team competences.

Hence, increasing emphasis is put on the development of management as it is seen as the key driver to induce organisational cultural change, to build up shared organisational values and to promote effective performance management. An established UN competency model suggests that core organisational values and competencies may only be induced by competent management. The model contains three core values, eight core competencies, and six managerial competencies, the latter playing a dominant role in the model as shown in table 7 below. 
Table 7: UN competency model

\begin{tabular}{|l|l|l|}
\hline Core Values & Core Competencies & $\begin{array}{l}\text { Managerial Competen- } \\
\text { cies }\end{array}$ \\
\hline Integrity & Communication & Leadership \\
\hline Professionalism & Teamwork & Vision \\
\hline Respect for diversity & $\begin{array}{l}\text { Planning and develop- } \\
\text { ment }\end{array}$ & Empowering others \\
\hline & Accountability & Building trust \\
\hline & Creativity & Managing performance \\
\hline & Client orientation & $\begin{array}{l}\text { Judgement/decision } \\
\text { making }\end{array}$ \\
\hline & $\begin{array}{l}\text { Commitment and con- } \\
\text { tinuous learning }\end{array}$ & \\
\hline & Technological awareness & \\
\hline
\end{tabular}

Source: Fernandez (2005, p. 233)

The model seems to acknowledge the middle role of managers between the organisation and the employee. Managers must fulfil contradictory roles as subordinates expect them to represent their interests upward to higher officials while higher officials expect them to present organisational goals downward to employees.

To be able to fulfil these roles equally well, managers need to possess managerial skills. Skills often referred to are a manager's ability to perform aspects of the job like handling interpersonal problems or handling the budgeting process. A group manager moreover needs to be able to handle group dynamics and has to know how to delegate and allocate tasks. Moreover, an important skill is to know how to empower and motivate others and how to build up trust.

To develop leadership and managerial capacity and to enable managers to successfully fulfil their role as a link between the organisation and the individual employee, the following trainings are offered:

- Management Trainings are targeting D1 and D2 staff and P-staff with supervisory responsibilities and focus on the development of leadership competencies.

- Department-based managerial Development focuses on providing specific trainings according to the needs of individual departments. The aim is to strengthen the skills of individual managers by taking into account the people and processes within a specific department.

- Supervisory skills trainings focus on the development of staff at all hierarchical levels who has supervisory responsibilities and it accompanied by a follow-up programme to reinforce the skills learned and to assess further needs. 
- Team based workshops aim to promote team work by introducing the concept of team work.

- Intercultural workshops are designed to raise awareness of the impact of diversity and aim to show how to work constructively with people from different backgrounds.

The plan for raising team awareness by team training was put into practice immediately as at the time of the interview interviewees in management positions were already engaged with management training in various forms:

There is an initiative which began in our HR department. Within the HR department there is section responsible for training and education and they have offered team building courses. These courses are offered in my sector so we need to spare 2 days for teambuilding. This is nothing new for me, it is the third teambuilding course I have taken [laughter] or management course. [...] the ones I took focused on psychological aspects. I recall a course which started with half a day of analysis of my own personality profile according to Jung. $[\ldots](\mathrm{P} 39: 17)$

Yes, it will be a team building training. We did a process analysis then we have some recommendation of the management and there was a lot of communication and so on and so forth and now we are taking all the groups sector of the trademarks in team building exercise, a training, then we will complement that by a management training where $\mathrm{XX}$ will take part and where we discuss all the issues we got from the teambuilding training. (P38:24)

I know that the UN actually has training on multiculturalism or so for managers on how to manage team diversity, cultural diversity I think is what they call it. They have a - I think it is a one day training which you can take but only if you are manager. (P47:138)

Oh yes, there has been some intercultural training recently but only for section and unit heads. But we all should receive the training material or at least have access to it. (P8:38)

The last quote above highlights the fact that all of these trainings were made available to staff in leading and supervisory functions only. However, the need for diverse trainings not only applies to management but also to all other levels of staff. This implies that both management and subordinates need to develop skills. Therefore, a further aim of management training is to enable managers to 
pass on their knowledge to their subordinates and to encourage them to participate in various trainings themselves.

One group leader described how skills learned in the course of training were passed on to subordinates:

Well, I have done some trainings with them...I tried to raise team spirit so that everyone knows that well...I need this person for that and in return this person needs me for something else. (P35:67)

Usually, management takes over staff development in the context of performance appraisal by assessing needs and wishes of individual employees. The focus of such appraisals lies on

- explaining organisational needs and goals and how to reach them

- working on individual needs such as training and skill development

- assessing career opportunities

The quote of one employee below illustrates the agenda of individual appraisals:

There are personal reviews. Tasks for the next year are set and at the end of the year one has a look whether or not the task has been achieved. Also some sort of career plan is discussed which I find very good. (P2:56)

\subsubsection{Criticism of management}

As in any other organisation, criticism of management is part of organisational life and was frequently expressed throughout the interviews.

One major criticism dealt with the effect of political job positioning of managers. This topic has also been addressed by Kofi Annan (2006) who stressed the importance of more transparency and quality of selection of management across all levels of the organisation. He emphasised the fact that only a more rigorous selection process combined with a clear statement of responsibility and authority of individual managers will increase their accountability. Moreover, he proposed to establish clear criteria for assessing the performance of managers.

In practice, however, empirical evidence shows that despite reform attempts, the general problem in hierarchical organisations (Gortner et al., 2007)is that a formal position in the hierarchy does not prove anything about a person's management abilities, still prevails.

The following quotes summarise the criticism of management: 
They recruit any managers, they are managers but they do not have real knowledge in the specific field. That's the actual problem. You get a manager who used to be responsible for, let's say, food and now he/she has to manage the print office. (P35:165)

In my view too many managers are sitting in the wrong places but these organizations have no influence on it because top management is solely politically appointed, this is one of the shortcomings in these organizations. (P29:64)

Ahm, the manager is most important and I think that's the problem because in the UN the managers are too political. There are all these principles like integrity, respect for diversity in recruitment but in the end it is more a political factor which is stronger and that's why in the end we have all those managers who are not good managers. (P43:12)

When speaking about their own managers, interviewees predominantly criticised the lack of decision making competency of managers. Criticism not only alluded to the long and cumbersome process of decision making which is led by rules and regulations but also to the lack of decision making competency.

The following quote criticises managers who did not make decisions and furthermore lacked in communication:

In this particular office the management was really bad. Some argued this was due to the fact that both chief managers came from West Africa. I would certainly not have said so but I am still convinced that they were extremely indecisive. They never made any decisions. Also, the communication did not work but I do not think this was due to the multinational environment, I think they were just happy to have this important position and they did not work on it any more. (P13:06)

Half of all interviewed persons concluded that managers are afraid to make decisions and hold responsible for them because of a fear of losing their good job position:

They [the managers] can not make any decisions or they do not dare to and they do not take any responsibility. (P16:51)

However, interviewees also reported positively about their managers. Some described good managers by referring to human qualities of the manager and by describing different skills which distinguish a so called 'good manager' from a 'bad manager'. Interviewees across all hierarchical levels defined a good man- 
ager as a person who is empathic, fair, encourages career development and motivates staff.

He is really good. It's not that I mean to say that he has so much experience as a manager but he is simply suitable for it and he has these human qualities and he learns. He adjusts, he is empathetic, he does not have prejudices, he treats everyone equally. I think he is the ideal boss...there are not many of this kind. (P43:32)

An intern in a completely different hierarchical position who spent 9 months on a non-paid contract also points to the human qualities of the manager as well as the appreciation of the work as done by a motivating manager:

Well, it came across that the work that I did, the texts that I wrote were actually used, and that I did not do a stupid or useless job. This was some sort of confirmation for me. At the same time they always told me what I did not do well. I had the feeling that I was taken seriously in any aspect. (P30:12)

Similarly, a manager expressed the importance of motivation and support being a supervisor of superiors him/herself:

The motivation is the crucial part. For each individual you have to find an individual strategy. It takes more time but if you don't do it they will not understand the same thing. Everyone can come to me and ask again to make sure we all talk about the same. (P18:06)

To reduce negative criticism, the UN reform, which was instigated in 2006 by Kofi Annan, targeted several topics a major one being the aim to improve the current management situation. Unfortunately, this reform could not be realised because of a negative vote of several member countries.

Resistance to change seems to be very strong and the vote against the proposed reform is probably the best example for this movement against change as captured by the interviewee below:

You have to manage change very very carefully in this organisation. You have to package it in such a way that it is not threatening the people; that they don't get scared and then they accept it. Because if people don't accept change it could be very bad. And right now, the $\mathrm{UN}$ is in a situation that people don't expect change. Even the coun- 
tries don't expect. You know, they just voted against the UN reform actually. The UN reform package did not pass. Everyone is talking about reform but the reform package did not pass because the developing countries voted against it and they had a majority because out of 191 countries they had 91 member states and it was a simple vote, every country has one vote and they have the majority there and they oppose it. (P47:66)

\section{Summary:}

Summing up this section on the organisational level of the United Nations System as well as on the role of management several conclusions can be drawn and will be explained in more detail in the following.

All United Nations organisations which were part of this study were characterised by a strong hierarchy. This hierarchy is reflected in the categorisation of staff members into different bands which comprise different levels of power as well as a specialisation of tasks. However, depending on the band, this specialisation varies. Band 1 staff, or general staff, predominately reported of routinetasks whereas Band 4 staff, or senior management, reported the opposite.

The strong hierarchy and specialisation of tasks imply that the formal structure of the United Nations Organisations under investigation does not incorporate a formal team structure. Apart from a strong hierarchy, interviewees also reported a strong bureaucracy with numerous rules and regulations and a high level of record keeping. This intense bureaucracy is explained as being necessary to assure accountability in a political, intergovernmental organisation whose specialised organisational units are financed by governmental money and/or membership fees. Hierarchy and bureaucracy are the main influences on organisational life as pointed out by the majority of interviewed staff. Amongst other, the personnel system especially has been described as being affected by hierarchy and bureaucracy. The recruitment process is very lengthy, contracts differ from position to position, promotions are based on seniority and not on merit, and a change of position involves the whole application process all over again.

To overcome hierarchic and bureaucratic hurdles, individuals start to break down boundaries by finding ways of communication outside the hierarchical structure and its rules and regulations. By doing so, networks and parallel systems to the official system evolve. The reasons for boundary spanning activities can be numerous, e.g. a lack of information or the need for swift decisions or actions.

The relationship between management and employees has been identified as being crucial. Management can be seen as the major link between employees and the organisation as the manager is the most immediate and tangible representation of the organisation in the eyes of the individual employee. The quality of 
the exchange relationship between an employee and his/her manager is pivotal in determining the employees future within the organisation (Sparrowe \& Liden, 1997).

Interviewees believe that a 'good manager' who motivates and empowers them is the key to successful group and team work. However, the criticism of management as being too politically appointed and the rotation principle hamper the relationship between employees and their superiors.

The following Network View visualises the relation of components and the 'groundedness' of each code in the data by quantifying how many quotations are grounded in a code. It also shows the number of links made to other codes, the so called density. The numbers in the brackets next to the characteristics represent groundedness and density:

Figure 7: Network view on organisational and managerial components

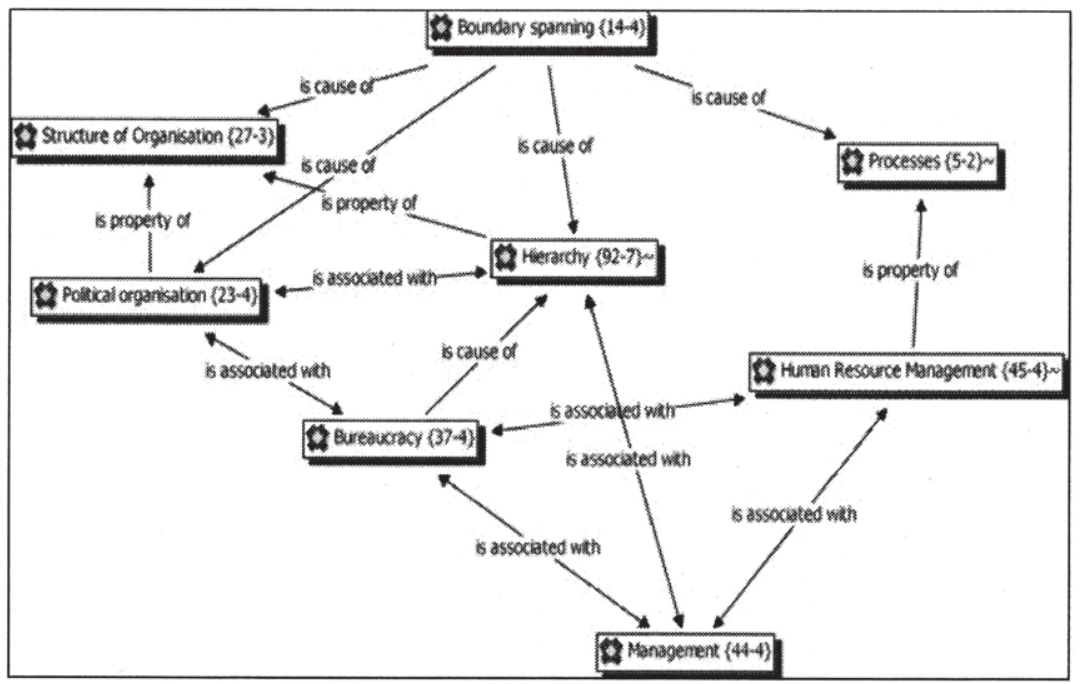

Source: Data analysis

Hierarchy lies at the centre of the network as it directly and indirectly influences all organisational components and processes. 


\subsubsection{Employee - Co-worker relationship}

The employee-co-worker relationship is at the core of group work as it is strongly reliant on social aspects and driven by individual attributes. Where the employee-management relationship contains elements of economic exchange (e.g. salaries), the employee-co-worker relationship is almost exclusively driven by social and psychological components of exchange. So far, data analysis revealed that this relationship is strongly influenced by organisationally induced factors. As explained earlier, the hierarchy of the organisation and the standardisation of tasks are the basis for the employee-management relationship and also for the employee-co-worker relationship. The categorisation into bands and the type of task an individual fulfils set the stage for group work or collaboration of individuals. The interaction with co-workers will vary as some tasks involve intensive communication and exchange, whereas others involve comparably little interaction.

Further data analysis revealed that on an employee-co-worker level diversity in its various forms has the strongest impact on interaction patterns and on group behaviour. Moreover, the interviewee's narratives unfolded on a continuum: on a general level, diversity in national origins of individuals and associated stereotypes were reported in a neutral way, meaning that these characteristics of coworkers do not impact or influence the overall work environment. However, when individuals talked about group work or collaboration, cultural diversity in its various facets was reported to be a strong factor of influence on work. Interviewees alluded to diversity in gender and age, diversity in working styles and language and their influence on the work environment. Further topics that were marginally mentioned by interviewees related to a different feeling of time, humour and different sources of conflict and ways to solve them.

Each of the nuances of diversity mentioned by interviewees are visualised in the network view below (figure 8) and will be explained in detail: 
Figure 8: Network view on employee-Co-worker relationship

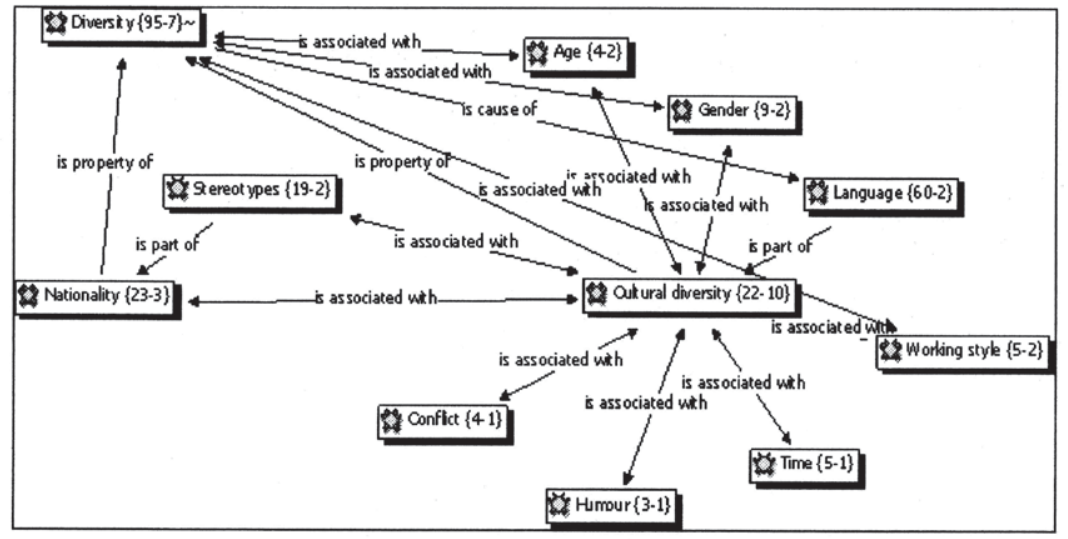

Source: data analysis

\subsubsection{Diversity}

\subsubsection{Nationality and culture}

The United Nations Organisation, by its very nature, is a multinational place as the name itself suggests. Subsequently, it is also a multicultural place with diverse ethical, religious and socioeconomic structures. As a consequence, encounters of diverse nationalities and cultures are a fact of daily work life. Culture according to Hofstede (1997) is conceptualised as "collective programming of minds" suggesting that from the time of birth values and beliefs specific to our own culture and society are programmed into our minds. Culture, furthermore, can be seen as a set of dimensions which determine our behaviour and patterns of interaction. Because the United Nations Organisation comprises 191 nationalities, a question to raise in this context is whether or not nationality and national culture matter to staff members. There are two views on when nationality matters in existing literature: one assumes that nationality does matter to employees because of different values and preferences with regard to management and leadership which are related to national and cultural background (see e.g.Hofstede, 2001; Schwartz, 1994; Trompenaars \& Hampden-Turner, 1997). The contrary view suggests that nationality does not matter because of other, stronger factors like age, gender or profession and education. According to the latter, people from different national backgrounds working at the same hierarchical level have more in common with each other than with people from their 
same cultural background. Both views are represented in the data which corresponds to a more recent and dynamic view of culture based on the political, economical and technical consequences of globalisation. This view alludes to a world community which shares a common set of values, attitudes, norms and behaviours (Bird \& Stevens, 2003).

According to the majority of interviewees national diversity per se is not salient in the work context. It is seen as a 'normal' component of work life. Moreover, interviewees relate nationality to foreignness and highlighted the fact that everyone in the organisation is a foreigner and therefore the foreignness is not salient. With the words of several interviewees:

For me, this multiculturalism is relatively normal. Because I have worked in an international field for quite a long time. (P13:48)

One thing that is really positive is that nationality does not play any role in mutual approach. Here, we are all foreigners. (P7:07)

You get used to it [multiculturalism], it is routine to sit in front of a person from India. [...] It is not like in a family but everyone who works here comes from a foreign country and we all feel bonded because we are all foreigners. Also the Austrians, for them it is just like they were foreigners as well. (P21:15)

The notion 'we are all foreigners' refers to the above explained view of a world community in which nationality does not matter. The interviewee's statements may derive from their interpretation of article 100 of the UN Charta which stipulates: 'In performance of their duties the Secretary-General and the staff shall not seek or receive instructions from any government of from any other authority external to the Organisation. They shall refrain from any action which might reflect on their position as international officials responsible only to the Organisation.' (Charter, ; UN, Chapter 15, Article 100).

An interviewee makes the point by referring to the United Nations as a 'common nationality':

There is article 100 of the UN Charta which says that we are no national representatives we are, so to speak, without nationality. The UN is our nationality. From the moment you work for the $U N$ you can not say any more that you are Austrian. You also have to sign something. 
You are allowed to vote but you must not carry out any political activities. (P40:12)

Other interviewees pinned down foreignness to the physical building of the United Nations Organisation. A number of interviewees described that the entrance into the building with all its security checks made them feel to enter another world. As expressed by two interviewees:

You need to show a passport when you want to get in and it makes you feel like you are in a different country. (P22:23)

We are not in Austria. (P7:34)

At the same time, however, more than three quarters of interviewees report of special care and attention which is paid to cultural differences and which result in increased awareness:

You need to be very considerate because everyone has a different attitude. You need to approach each other slowly and carefully and you need to reduce prejudices. This may sound negative but in real life it is not like that. You may have to invest a little bit more time but you can learn this. (P5:06)

You need to have a lot of respect. You have to learn not to hurt feelings and not to be too fast with judgement. [...] An open attitude is the best thing which you can bring in such an organisation. (P25:06; P25:07)

Generally speaking I would say that the people here are very open minded and maybe they are a little bit more sensitive because of the fact the everyone is a foreigner. Not to take everything for granted. (P46:71)

\subsubsection{Stereotypes}

The point at which nationality and cultural diversity definitely become salient to the interviewees is when direct interaction with people from other cultures takes place. At this stage, the previous notion that nationality does not affect interactions loses power as interviewees also started to include racial and ethic stereotypes in their narratives. Racial and ethical stereotypes are pervasive, persistent, frequently negative and often self-contradictory. Most of us hold stereotypical views of other races or cultures and ourselves. Moreover, such stereotypes ex- 
hibit remarkable stability over years and personal experience is not necessary to form stereotypes. (Alderfer \& Thomas, 1988)

The following quotes best capture these insights:

The South Americans are usually very very funny and easy going but also in Asia it depends on where the people come from. We have very untypical French guys, for example, French people who do not enjoy eating and drinking wine. Suddenly you see that there are not only French guys who love to eat. And then one stops saying the Italians are like this, the French are like that, a Latin American is like that. (P6:15)

We do have a couple of Americans who do not care about anything at all. [laughter] (P8:14)

The usual verbal teasing just like between Austria and Germany. (P17:60).

However, stereotypes are not solely the triggers which make employees consciously notice national and cultural diversity of their co-workers. The data analysis also showed that national diversity becomes salient when related to team and group work, in the following quote especially with regards to language (language will be discussed in more detail later on):

The only time I think about nationality is when they don't speak English well and then I start to think why they don't speak English well. (P28:13)

\subsection{Excursus: Working in the field}

All of the interviewed people self-reported that they notice stereotypes when working in the field and equally strongly experienced cultural diversity. To work in the field in United Nations jargon means to work outside the headquarters as an expatriate. Work in the field usually involves work in developing countries and is seen as a means for transferring the headquarters' knowledge to the field. When working in the field, interviewees had to cope with host culture work environments which differ to Western standards with regards to productivity and performance as well as punctuality, decision making and concepts of authority. Acculturation

Acculturation strategies to different cultures have been extensively discussed in the relevant literature. Berry (1984) distinguishes between two types of accul- 
turation based on maintenance of cultural identity or building up of relationships with other groups. The outcome of either acculturation process has been conceptualised as intercultural adjustment (Ward, 1996).

The data analysis also revealed that interviewees chose either of the two acculturation processes. The quote of the following interviewee can be categorised as an approach of maintenance of cultural identity which however, does not appear to be easy:

I try not to acculturate but you get slower because everything takes much more time. You have another problem for example in Africa, they never say no, we can not do it. For us it would not be a problem, just tell me that you can't do it and I will find another solution. In Africa they don't say it and work simply does not get done. That's a huge problem and it happens all the time. (P16:17)

Contrarily, the next interviewees describe an adaptation to the behaviours in the host country and the building up of relationships with other groups according to their cultural standards:

With regards to the working style, it is a learning process: you have to learn to deal with different pattern of behaviour. The people in my office are very much process orientated. So you try to be less impatient and allow others more time. (P14:52)

You accommodate to how it works in a country ... that an agreement on the phone is not taken seriously, or when organizing an event you do not take it too seriously that every 100 percent is fulfilled but you try to get it done and make it work. (P13: 19)

\subsection{Feeling of time}

Respondents who have had work experience in the field repeatedly reported of a different feeling of time as a major issue when working in another country. Interviewees described a divers understanding of time which is in line with existing research about different understandings of time among cultures (Trompenaars \& Hampden-Turner, 1997). The latter distinguish between a monochronic time approach which suggests that time is conceived as a line of sequential events and a polychronic time approach which allows numerous activities at the same time. Austrians are categorised as having a monochronic understanding of time and hence, are insistent on punctuality. 
Fink \& Meierewert (2004) discovered two types of time behaviours in East and Central Europe: slow speed of task solving and extreme length of negotiations and decision making processes. They explain these types of time behaviour in a tendency to work in collectives rather than teams and in the priority setting power of supervisors rather than employees.

Both types of behaviours are also supported in the data:

One guy in our office sometimes really gets upset because reports are not finished despite a deadline. But his superior says that he has talked to some other people and they have great input and we should wait for it so in the end it can take weeks until we finally get the report. Of course it may be a really great input but a deadline is no deadline any more and this is sometimes difficult. (P14:63)

...to understand that by tomorrow does not mean by tomorrow but by someday. If they say maniana it means by sometime and you must not expect that it means by tomorrow. (P13:06)

\subsubsection{Gender}

One of the most problematic stereotypes for organisations in general is the gender stereotype. Since the early 1970 s when researchers conducted the first studies on stereotypes they have essentially stayed the same up until now. These studies have determined that successful management is related to traits and attitudes which are similar to those generally ascribed to men, that is, qualities such as leadership ability, competitiveness, self-confidence, ambitiousness, and objectivity (Brenner, Tomkiewicz, \& Schein, 1989; Schein, 1975). More recent research describes good managers as possessing less masculine characteristics than in past decades; however, it still indicates that both men and women of varying age, education, and work experience predominately refer to masculine characteristics when describing good managers (Powell, Butterfield, \& Parent, 2002). Considering the number of woman in the workforce, they are severely underrepresented in managerial jobs and top-level positions. This stereotype is also true for the United Nations Organisation. A simple demonstration of the gender problematic can be found at the very top level of management: since the founding of the United Nations in 1945 all Secretary Generals were male. The same pattern exists on the Directors level and on the Professional level.

United Nations staffing policies aim at an equal participation and representation of gender across all hierarchies. A 50/50 gender distribution in all posts in the professional category as well at a director's level and above is a goal set by the General Assembly (Fernandez, 2005). To meet this goal the following measures have been taken: whenever a job vacancy is announced, priority is given to seek female staff within the organisation as well as outside of the organisation. Fur- 
thermore, accelerated promotions of female staff members apply until the goal of gender equality is met. Both measures only apply provided the female candidate complies with the requirements for the vacant job with regards to qualifications and experience.

In reality the gender stereotype appears to be a delicate topic. As explicated by two male interviewees who describe a reversed stereotype:

I can think of an example in which I personally was the bogeyman. A P5 post was advertised, a directors post, and a woman applied for it. We all agreed that this woman was not qualified for the job and I also communicated it this way. And imagine, in the end I was drawn in front of the committee in NY and in NY they called me an adversary of women just because I said that this women was not qualified for the post. (P31:47)

Yes, there are gender issues. The posts are split 50:50 but it will take some time until senior functions will be carried by women. The first woman who applied for a senior post was too young. There were few female candidates because men have been favourites. At the moment, women are clearly privileged and so it is difficult for men. (P49:103)

However, gender stereotypes tend to favour women when they are being considered for 'women's' jobs or for 'women's tasks as illustrated by the following quote:

I work with 25 women because the jobs are very administrative and need attention to details. Women are better in this than men. (P18:23)

\subsubsection{Language}

The United Nations Organisation uses six official languages in its intergovernmental documents and meetings: Arabic, Chinese, English, French, Russian, and Spanish. The Secretariat uses two working languages: English and French.

The daily working language of the sample predominately is English which is to 47 out of the 50 interviewed persons a foreign language.

Communication is a vital and major part of team and group work. At the same time, one of the main barriers to communication is that individuals speak different languages. Therefore, a common language has to be used which most of the time is not the native language of the members of the group. Several researchers have noted that the level of language proficiency effects team and group work and demonstrated its crucial role. Findings indicate that language has a consid- 
erable impact on socialisation processes and that the use of a common work language may result in unsuccessful communication and ambiguity (Feely \& Harzing, 2003; Henderson, 2005; Marschan, Welch, \& Welch, 1997).

An Interesting insight of the current study is that only non native interviewees reported about this very language barrier and about its consequences on communication.

The comment of a native speaker:

Language is not a problem for me since I am native speaker, I can't tell how non-native speakers feel about it. (P20:35)

Compared to the remarks of non native English speakers:

It does make a difference to communicate in your non-native language all the time. Even tough you know English very well it is not your mother tongue and you can not express yourself like in your mother tongue. So it is more difficult to communicate. (P13:23)

What I notice is the language barrier. The fact that people come from many different countries is not beneficial for communication. With native speakers who fluently speak the language it is sometimes a problem - at least for me. (P2:06)

Sometimes things happen and you think that the collaboration does not work a hundred percent. These are often language barriers and communication problems. (P5:61)

To overcome these barriers, the same interviewee described to choose simple language to make sure that the content of the communication becomes clear:

You are definitely not faster, it takes some time and in some cases you use a simple level of communication. It takes longer to transfer knowledge or to tell someone your wishes. (P5:27)

Others narrated that technical language serves to overcome barriers. Technical language as an element of a professional culture which is characterised by a specific type of vocabulary helps to at least partly overcome language deficiencies: 
We communicate on a very technical level....there are certain expressions which we use and so on this very technical level the communication works. However, when it comes to discuss problems or when we say, well the technical part is ok but maybe we should try it differently next time, because this might me more effective you need to switch to a meta-level and then, yes, then I think communication problems arise, when it comes to reflecting the work. (P45:36)

The last part of the above quote alludes to the proficiency of English. Although excellent knowledge of English is presumed, the data analysis indicates that English as a shared language does not ensure efficient communication. Different interpretations of underlying meanings based on different cultural norms are the source for misunderstandings and in the worst case even conflicts.

Sure, he [a Chinese superior] is very hard to understand because his English is so Chinese. But one can interpret what he tries to explain. I myself also make mistakes because I realize a couple of days later that he meant it differently and that he was right. (P24:42)

Slowly a couple of things became clear to me for example that specific situations of conflict are a result of language problems. On one hand it is the careful formulation in German language. Then the sender of the message does not transfer what he intends to because his English might not be as good so he can not transfer what he wants to say from one language to another. (P11:06)

However, interviewees are aware of the fact that communication in a language other than the mother tongue hampers their ability to express themselves. The notion that speaking in a non native language feels like speaking on a different level has been supported by several interviewees as illustrated by the following quotes:

You always communicate on a different level. The choice of language and what you try to communicate is not clear, and you always have to move to another level. You can be really fluent in a language but you will never be able to bring it to the point just like you can do it in your mother tongue. (P10:45)

It becomes a barrier at the moment. IF I do not know a specific word that's my own fault. The advantage of working in a foreign language 
is that you can use a third room...we come from the Philippines, Burma and Germany, and when we speak in English we all speak in a foreign language. (P46:35)

The empirical findings above illustrate that communication at the workplace takes place at different levels which is depicted by layers of language. These layers are interconnected, yet, each single layer may be a source for communication problems. Everyday spoken and written language used for communication between individuals and units and for external communication. Company speak refers to a specialised internal language of a whole organisation or company. Technical industry language refers to jargons such as 'Eurospeak', the jargon of the European Union (Neyer, 2004).

The three layers are shown in figure 9 drawn from Welch, Welch \& Piekkari (2005):

Figure 9: Layers of language

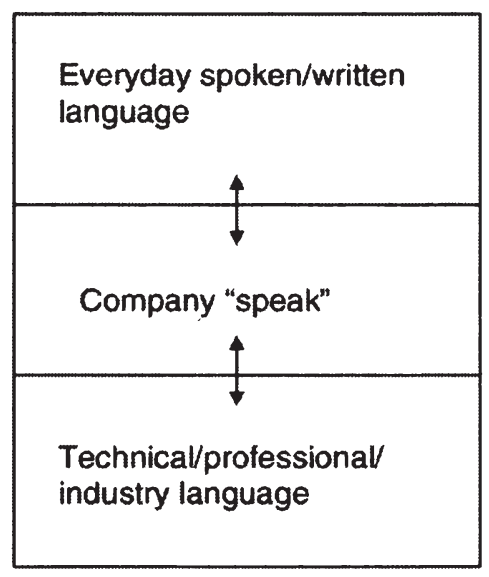

Source: Welch, Welch \& Piekkari (2005)

\subsubsection{Faultlines}

Traditional research into diversity has stipulated that work group diversity may affect performance either positively or negatively. Among the positive aspects of diversity research suggested increased creativity and innovation attributed to the work process. The negative aspects alluded to more complexity in the decision making processes and hence longer work processes. These findings are also in line with other research on diversity and performance as summarised in the 
literature review (Bowers et al., 2000; Webber \& Donahue, 2001; Williams \& O'Reilly, 1998). In a similar vein, data analysis revealed the positive and negative side of diversity.

The words of two interviewees give account to the positive and negative effects of diversity on performance:

Multicultural: multi-faceted but processes take much longer

Monocultural: fast results because there are less potential sources of misunderstandings but there is also less innovation. (P14:39)

In my view monocultural teams are faster in fulfilling a task because a lot of hurdles drop out. However, multicultural teams have the advantage that different perspectives can be integrated into the workflow and as a result the quality of work can be increased. (P19:55)

However, as also elaborated in the literature review, diversity may affect group work not only either positively or negatively but diversity is multifaceted and multidimensional. Hence, the effects of one dimension of diversity may be contingent on diversity on other dimensions. This highlights the notion made in the literature review that the different kinds of diversity, respectively demographic, job related and individual diversity may not be separated from each other but rather conceptualised in one framework. The term 'faultlines' which was coined by Lau \& Murnighan (1998) refers to combinations of dimensions of diversity which build a basis for differentiation between subgroups. According to the authors, 'faultlines' are 'hypothetical dividing lines that may split a group into subgroups based on one or more attributes.' (Lau \& Murnighan, 1998, p. 328). These faultlines or subgroups based on different attributes were also found in the data. For example, cultural diversity was also reported in connection with other types of diversity such as gender, race, age and functional diversity.

The following quotes for example illustrate the interplay of race, hierarchical position and age:

Especially among 'white' senior managers you can still feel that they feel superior and they also show it even if not directly. Younger colleagues are mostly more horizontal, more sensitive and more open towards other cultures. (P49: 44)

There are people who discriminate themselves. I have African colleagues who do it. But also: woman have to work twice as hard as men to be the same. (P25:15)

Let me take as an example India in 2001 . We had difficulties to get a woman, our team member, into the tent. There were doctors from In- 
dia who did not accept that women sleep in the same tent as men. But there was no other alternative and we needed these women in our team to communicate with the weak population, weak in this situation, the children and women in a natural catastrophe. So these are hurdles which one has to overcome. Nationality is a sensitive area, gender is another sensitive area in teambuilding. (P42:36)

There is sometimes some sort of a generation conflict. This is not so intercultural, but I am the youngest in my immediate environment. I am 20, the others are 35 and then 45 and older. We all have a different view of the world. It is not so much age it is more blindness by routine. (P11:34)

Faultlines also emerged based on functional diversity and age.

As in many hierarchical organisations, seniority is directly linked to age as the process of promotion is based on the number of years with the organisation and not on merit. A the same time however, the assumption that a certain age range of a person is related to the person's physical, psychological, and intellectual capabilities is also true for the United Nations organisations. These two assumptions create a faultline which suggests that older employees are more reluctant to induce changes than younger employees and that older employees do not possess the skills requested for a changing work place. As reflected in the interview data:

If I wanted to discriminate against, I would do it via age in the sense that the UN has changed a lot. Let's say it used to be an organisational forum to build and maintain a dialog between east and west while today UNOPS and UNDP mainly provide development aid and post crisis aid, so the skill set has changed significantly. Nowadays we actually need more engineers, business administrators, lawyers and not so many international relations people any more. People here hold positions which are rather manager positions than international relations positions, and you often notice that they lack management skills. When these people started 20 years ago the UN was completely different. And I would not want to accuse anyone. (P44:31)

To bring young blood at a professional level to the organisation, the Junior Professional Officer (JPO) Programme was introduced in 1972. This programme which runs for 2 to 3 years provides young graduates the opportunity to gain substantive job related skills and knowledge for future work in senior positions 
within the United Nations organisations. The JPO's are seen as a promising source for changes within the system:

JPOs defenitely have a more progressive view than senior management. (P49:109)

I have to admit that JPOs are model students when it comes to efficiency and efficient costs savings. (P10:40)

Another source for progressive ideas is the UNIDO internship programme. Young graduates or students who are about to graduate work on a 6 month basis as unpaid interns and conduct research in areas of direct relevance to the organisation. The students get the opportunity to gain work experience in an intergovernmental organisation while the organisation can benefit from fresh ideas at no cost.

\section{Summary}

Concluding, diversity in its various facets is a major component of group work. The charter of the United Nations manifests national and gender diversity and the organisational structure naturally creates functional diversity. As in other organisations, general stereotypes on nationality and gender also exist in the United Nations Organisations. When working in a group or in collaboration, national and cultural diversity become salient to individuals and different strategies are used to cope with diversity.

The interview data revealed that the use of a common, foreign language as the main means of communication creates barriers to team and group interaction. Communicated messages can become ambiguous and subject to individual interpretation and in the worst case result in conflict. Strategies to cope with this communication barrier are the use of simple language or technical language; both minimising the propensity for misunderstandings. Interviewees describe being aware of the fact that communication in a foreign language restricts their abilities to express themselves as compared to communication in a native language.

Faultlines or subgroups are the result of all the different types of diversity and based on different attributes. However, not all faultlines become salient. It depends on 'whether features of the context in which individuals operate highlight the faultline' (Polzer, Crisp, Jarvenpaa, \& Kim, 2006). In this research setting the context is characterised by hierarchy and national diversity which highlight faultlines. 


\subsubsection{Individual}

Each individual carries a different culture compared to another individual. Because of its uniqueness, individual culture may be compared to a fingerprint. But how to describe individual culture?

The culture of an individual according to Krentzel (1999) comprises several components: national culture, ethical culture, sex, age, social background, social class, education, profession, and workplace. All of these components vary from one individual to the other. Moreover, some components are more salient than others. For instance, national and ethical cultures are the most salient characteristics of individuals and learned from childhood. Education and profession are choices that are made at a later stage in life and are easier to learn and to change. However, social class and social background play a huge impact on these choices.

Considering the uniqueness of each individual's culture one might argue that it is impossible to generalise characteristics of several individuals.

Yet, the final notion 'it all depends on the individual' was a very clear and strong pattern that emerged throughout the data analysis and hence is explicitly grounded in the data. Data analysis revealed three facets of the individual which are perceived to have the strongest influence on group work, namely the personal background of individuals, personality and motivation. Each of the facets will be explained in detail and illustrated with quotes. Subsequently, a network view will be presented and further propositions will be enunciated.

\subsubsection{Personal background}

Individual staff in the United Nations Organisations can be characterised as being very internationally orientated and interested. More than $80 \%$ of the interviewed staff has lived and worked in foreign countries either prior to the work with the United Nations or as part of it. A considerable number of staff have grown up in a multicultural family or in different countries and speak at least one foreign language fluently. Other staff have not had international experience and therefore were keen to work for the United Nations to gain this experience. All of these backgrounds however comprise an affinity for other cultures and nations as also explained in the interviews:

I am used to working in an international environment which makes work much more interesting because you learn and hear from the world and not only from a small or specific area. You can overlook barriers - this makes life simply more interesting for me. And it helps to see world politics with other eyes or from another angle and not the way the media and radio present it. (P2:17) 
I always wanted to work in an international environment because I simply find it great to work with people from different nationalities. Besides, I always wanted to speak other languages so during a regular workday I speak German, English and French. (P9:23)

I think the people who start working for the UN bring many good qualities. They know many languages and on average they are much more international and culturally aware than in a national company. So if you work for the UN you know that you have to be open minded towards other cultures and I love this aspect. (P40:36)

\subsubsection{Personality}

Data analysis showed in line with existing research the importance of personality as an influential factor on group work. Interviewees attributed far more importance to a group member's personality than to his/her national and functional background. Personality as described by the sample comprises the character of individuals, and whether or not someone is empathetic and a team player.

Interviewees explained this point as follows:

I would say you adjust to people, and in my view that does not depend on nationality. If I work with someone, I will get to know this person and adjust to this person. And just as I said whether this person is from Chile, Cuba or somewhere else does not matter. (P3:06)

It is completely irrelevant where people come from. Sometimes you speak of someone as 'the Indian' or 'the Japanese' at least you know who you are speaking of. But personally for me it does not make a difference where people come from. It more depends on personality than on nationality. (P11: 94)

Well, my personal feeling is that it does not matter which nationality someone belongs to, the character counts. (P12:14)

To start with I would like to say that it all depends very much on the person, on the individual, that's most important. How individuals interact is culturally coded and many cultural factors play a part. Team members subconsciously handle this. (P15:11)

The problems at work don't come from nationality. They could be linked but I think it comes more from personality. (P18:25) 
I am colour blind. I see the person, not the nationality. (P25:11)

In my view it does not depend on nationality. Of course you work with some people more easily than with others but this depends more on personality than on nationality. (P29:31)

\subsubsection{Motivation}

Individual motivation is a further crucial component of group work and directly influences performance. Fink \& Holden (2007) relate the concept of motivation to three theories of identity established by Jiménez at al. (2004) which are: instrumental theory, civic theory, and cultural theory. Instrumental theory suggests that perceived personal interests create identities. Civic theory argues that identities emerge if actors within a group collectively develop rules and goals and share the same values. Cultural theory suggests that identity is based on cultural heritage, common language, symbols and national networks. Fink \& Holden (2007) argue that extrinsic motivation fits with instrumental theory as wealth is an important part of this theory. Intrinsic motivation is argued to fit with cultural theory as shared cultural heritage, language and symbols play an important role. Research also suggests that the collection of tasks that comprise the job influence work motivation (Perry \& Porter, 1982).

In a similar vein, data analysis revealed a positive relationship between the hierarchical position of interviewees (and respectively the type of task they fulfil) and their perception of motivation. High ranking staff commented on intellectual stimulation and intellectual freedom as a source of intrinsic motivation. Work is perceived to be influential and to make sense. With the words of interviewees:

You work for intellectual stimulation. You see what happens and how it works out and that rewards you. (P20:33)

What you find here is absolute intellectual freedom and that's what makes work here so special and for sure is the main part of motivation. There is no other job in which you have so much intellectual flexibility, there are hardly any boundaries. No matter what you can suppose it as long as the frame conditions are fulfilled. Because there is need in development, in the area we work. And that's what makes work here so special. (P29:43)

Repetitive tasks or very well structured tasks on the other hand are perceived as being a source of demotivation. Therefore, staff strive for job variety as illustrated by the following two quotes: 
Well, because you do not have the global view which is not possible because of daily routine, you quickly just focus on this part and lose sight of everything else and this is for sure a source of demotivation. (P48:61)

My motivation is the variety because I don't solely work for XX but also for someone else. The aim of my position was to get to know everything. To do my own work really well is part of my motivation. There are different tasks, sometimes you can chose what you like to do sometimes it is not so exciting. (P11:127)

Some interviewees very honestly remarked that the high level of remuneration and the generous benefits are a further source of motivation. This kind of motivation relates to extrinsic motivation and instrumental theory of identity. In the words of two respondents:

It is very simple - I earn double of what I earn at University. (P24:08)

One of the major reasons for motivation is the fact that the pay is tax free. I would say everyone who is honest would have to name this reason. I would have to have super super job somewhere in the top level management of Siemens to earn so much money. (P9:23)

However, some interviewees commented critically on the negative sides of high remuneration. In a worst case scenario the motivation to work shifts to a motivation to simply keep the job and its benefits.

I think it all started when they set up the system. Specifically the HR system with the benefits and everything. (P47:98)

You lose that vision very easily. I think. Especially if you are concerned about the benefits like I need to stay because of the pension or they pay my kids the tuition at the school, I mean it is very tough. (P47:106)

\section{Summary:}

Summing up, the individual and his/her personal background, motivation and personality impact on the overall work situation and especially group work.

The personal background of most interviewees is very similar with regards to internationally orientated education or work experience. Moreover, the com- 
ments on motivation clearly show that the main source of motivation comes from the feeling of interviewees being part of an organisation with global influence. However, on a daily work basis this motivation may be strongly influenced by job characteristics, including tasks. The latter ones are directly related to the hierarchical position, hence, senior level staff report intellectual motivation while general staff find motivation in a variation of tasks. Some young interviewees criticised the obvious motivation by remuneration and benefits which due to social desirability bias supposedly has not been self-reported. It appears that the role of personality is very decisive. In line with findings in literature it can be assumed that specific personality traits are supportive of group work and collaboration.

Figure 10 below summarises the main components of group work in a network view.

Figure 10: Network view on components of group work

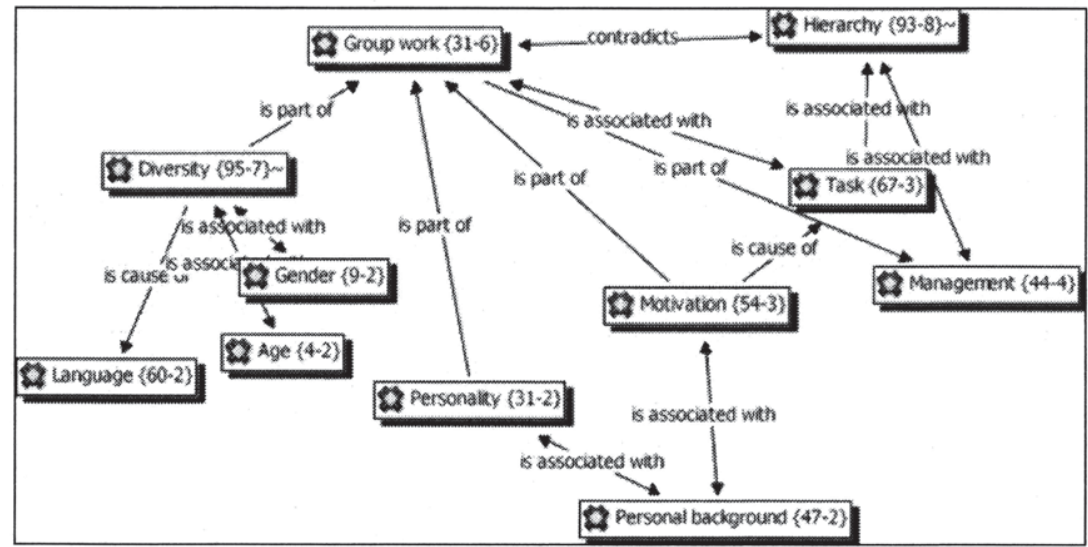

Source: Data analysis 


\section{Model and Propositions}

\subsection{Introduction}

The previous chapter presented results of the empirical analysis of 50 interviews conducted in five Organisations of the United Nations Organisation. In the course of constant comparison and a deep analysis of interview data, pivotal patterns which helped to answer the two research questions of this study emerged. These patterns were grouped into categories which depict the factors of influence on the work environment. The research questions will again be presented and each of the categories that have been developed will be discussed in detail. Furthermore, a conceptual model of group work in an intergovernmental organisation will be presented as well as propositions which have been deduced from the empirical findings.

Research question 1: What kind of team/group work exists in the United Nations?

Research question 2: What are the factors of influence?

The answers to the two research questions unfolded themselves in a process. The vantage point being an assessment of what kind of team/group work is practised in the United Nations Organisations under investigation. Data analysis revealed a typology of group work that is grounded in the interview data and directly related to the developed categories. The typology comprises:

- task forces described as a temporary group that are given a specific problem to solve,

- project groups which carry out specific projects within a given time frame, and,

- collaborating individuals which predominately work on individual tasks in collaboration with co-workers.

Moreover, the analysis in the course of answering research question 1 revealed a complex set of factors which influence the work environment. These factors were reduced to three main categories as shown in figure 6 and explained throughout chapter IV. All of the categories have been identified as being major and intertwined influences on group work and collaboration. The three main categories are:

- the organisational context (organisational structure and culture),

- the relationship between managers and employees as well as the relationship between employees and co-workers, and 
- the individual and his/her personal background, personality and motivation.

Lastly, boundary spanning activities have been identified as a moderator. The empirical analysis furthermore showed that there are mutual causal relationships between the factors of influence and that these factors are highly interdependent. An integrative view of all factors of influence leads to a conceptual model of group work in five Organisations of the United Nations Organisation which are illustrated in figure 11.

\subsection{Model}

The model shows at the very top the formal structure of the United Nations System as an overarching element which directly influences group work. The circle in the middle of the model represents the group which best can be described by the relationships between employees and managers and the relationships between employees and co-workers. The position of the management on top of the individual aims at showing the closeness of management to the organisation as well as the position of power of management. Ultimately, the relationships between individual employees are closely linked to the individual characteristics of employees comprising their personal backgrounds, personality and job motivation. Whilst the individual can be seen as a major component of group work, the boundary activities illustrated by arrows represent the moderator of group work. Lastly, all of the described properties of group work constitute the culture of the organisation.

The properties of the model have been described in detail throughout the empirical analysis in chapter IV. The next part of this chapter aims at discussing the propositions which can be deduced from the underlying empirical findings of the conceptual model. 
Figure 11: A conceptual model of group work in five UN Organisations

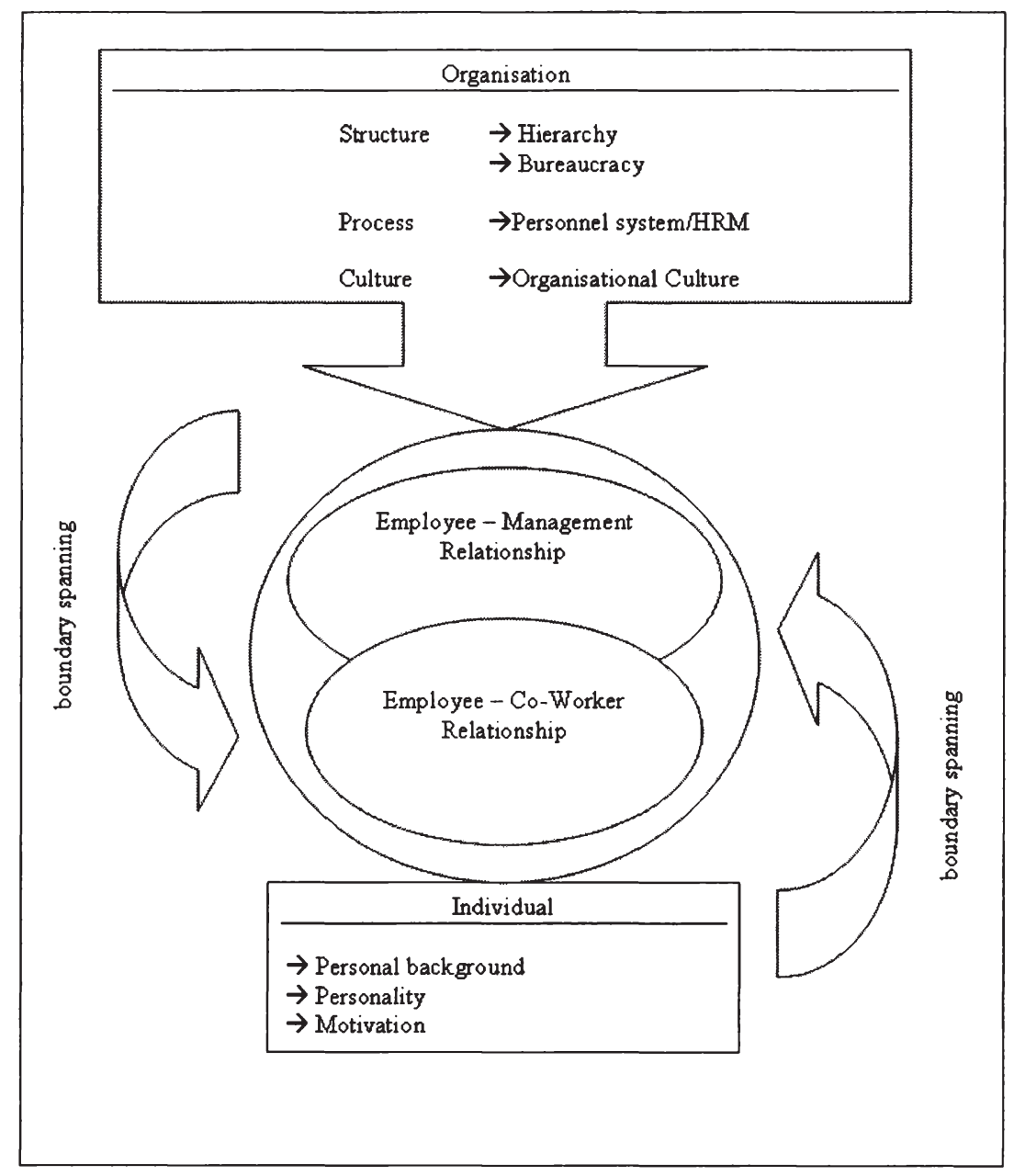

Source: data analysis 


\subsection{Propositions}

The following propositions are deduced from and grounded in the data and address the factors of influence on group work and the barriers for team work in the context of an intergovernmental organisation, respectively five Organisations of the United Nations Organisation. The propositions will be developed according to the categories of influence which have been identified throughout the data analysis.

\subsubsection{Organisation}

Data analysis showed that the typology for group work is determined by the structure of the organisations under investigation and their culture. In line with existing research (e.g. Rousseau \& Fried, 2001) this research has shown that the description of the context of a particular organisation is vital to understand underlying processes and phenomena.

Hence, in the following propositions with regards to the organisational structure and culture as a factor of influence comprising the context of the organisation will be discussed.

The strong hierarchy and specialisation of tasks implies that the formal structure of the United Nations Organisations under investigation is not yet compatible with a team structure. The concept of hierarchy and the concept of team work are found to be mutually exclusive in this specific context. However, there is evidence grounded in the data that group work as per definition is incorporated in the structure and actually performed. Nonetheless, the structure of the organisation presumably is stronger than the structure of the group. This shows that it is in the hands of the organisation to establish structures that allow for group work. As a consequence, it can be assumed that the same it true for team work provided that the strong hierarchy will be flattened.

The propositions aim at underscoring that hierarchy and bureaucracy, which are both major components of the organisational structure of the five organisations, and which were found to challenge the introduction of teams, should not be regarded as a barrier. It is rather suggested to use the advantages of these major components as a tool which has the potential to support the introduction of team structures.

Proposition 1: The hierarchy of the United Nations System challenges the introduction of teams and overarches the work of groups and individuals.

Proposition 1a: Hierarchy should be associated with responsibility rather than with restriction. 
Proposition $1 \mathrm{~b}$ : Hierarchy should be seen a factor of stability for individuals rather than an element of power.

An intensive bureaucracy is explained to be necessary to assure accountability in a political, intergovernmental organisation whose individual organisations are financed by governmental money and/or membership fees. Moreover, the rules and regulations offer stability and predictability. However, the plethora of rules and regulations was reported to be a factor which limits group work, the reason being that individuals may only act if authority is granted. The latter ultimately involves numerous administrative tasks which are described to take up a major part of individuals' work responsibilities and in turn keep individuals from working collectively. On the other hand, interviewees reported that bureaucracy is the only stable factor in a frequently changing environment which demands lots of flexibility from the individual. Moreover, the rules and regulations help to neutralise diversity in the sense that they can be learned and understood by everyone and regulate in such a detail that a misinterpretation becomes less likely. If bureaucracy is seen as a factor which offers consistency and helps to deal with diversity it may also be regarded as a means to facilitate group and team work by reducing complexity in a multicultural context.

Proposition 2: The bureaucracy of the United Nations System overarches group and individual work.

Proposition 2a: Bureaucracy assures accountability.

Proposition 2b: Bureaucracy is associated with responsibility

Proposition 2c: Bureaucracy offers consistency in a frequently changing environment.

Proposition 2d: Bureaucracy is a factor that neutralises diversity.

It is also suggested that the Human Resource Management should be adapted for a team and group based structure.

The Human Resource Management of the United Nations Organisations studied is depicted by three salient factors: firstly, there exists a variety of different contracts for staff that are categorised in the same band and fulfil similar tasks. This triggers inequality and resentment between individuals. A streamlining of these contracts would not only facilitate bureaucracy but also reduce inequalities. Secondly, another distinctive aspect of the Human Resource system is the performance appraisal system which is solely based on individual evaluation and not on group evaluation. A change towards a group based performance evalua- 
tion would be highly recommended to improve group spirit and effort. Thirdly, job rotation involves a regular change of positions and locations of individuals. Rotation negatively influences the identification of individuals with a group. The rotation principle may not be changed. However, rotating staff should make sure to build up on existing group structures and norms rather than to change them and reduce the identification of individuals as a group.

Hence, the following three propositions can be deduced from the findings:

Proposition 3: In a hierarchical context, the Human Resource Management needs to be adapted for team and group work.

Proposition 3a: A streamline of contracts among staff with same functions and responsibilities reduces inequalities.

Proposition 3b: A performance appraisal system which is based on collective performance evaluation encourages group and team efforts.

Proposition 3c: The rotation process needs to respect group and team structures to keep the identification of individuals as a group and team.

Another aspect of HRM, the personnel development system, has been described as being very competitive with regards to job promotions. The latter ones are dependent on available plan positions which themselves are subject to geographical filling. Furthermore, a promotion within the hierarchy depends on seniority and not merit. It is strongly suggested that an adapted personnel system should also consider skills which enhance and support group and team work as a factor for promotion.

To overcome hierarchic and bureaucratic barriers, the organisation and individuals break down boundaries and span new ones. On an organisational level breaking down of boundaries is being done by raising the awareness of the importance of group and team work and by holding respective training. Individual boundary activities create networks beyond hierarchical structures as well as parallel systems to the official system. Boundary activities should be encouraged as they increase communication and effectiveness. Boundary spanning activities may be seen as new structures for cooperation between individuals which form the ground for team and group structures.

Proposition 4: Boundary breaking and boundary spanning activities positively enhance work on an organisational and on an individual level. 
Proposition 4a: Boundary breaking on an organisational level is a system improving strategy.

Proposition 4b: Organisational boundary spanning creates an awareness of the importance of team and group work and sets the formal steps which enable group and team work

Proposition 4c: Boundary breaking on an individual level is a supportive practice to deal with the system.

Proposition 4d: Individual boundary spanning creates a parallel system to the formal structure which is based on cooperation and communication.

\subsubsection{Employee - Management Relationship}

The relationship between management and employees has been identified as being crucial for group work. Management can be seen as the major link between employees and the organisation as the manager is the most immediate and tangible representation of the organisation in the eyes of the individual employee. The quality of the exchange relationship between an employee and his/her manager is pivotal in determining the employees fate within the organisation (Sparrowe \& Liden, 1997).

To improve the quality of the relationship between managers and individuals, it is important to place skilled managers in these crucial positions. Therefore, it is vital to appoint managers, who ideally possess of political, economical and social knowledge and skills. The 'right' managers are task- and relationship orientated and in a position to induce changes on an organisational as well as on an individual level. Subsequently, they are also in a position of power to set the grounds for team work. Moreover, social skills will help to establish team and group awareness. For instance, the quality of management with regards to trust and motivation directly influences the propensity of individual employees to engage in group and team work. A symptom of low trust can be seen in the norms of communications that group members establish to protect themselves. An example of this behaviour is reflected in the use of emails: the sender copies a wide range of recipients on the message to make sure that all people up and down of the hierarchy are aware of the content. Moreover, the sender also passes on responsibility by acting this way.

Proposition 5: The role of management is a crucial link between the organisation and individuals 
Proposition 5a: Management is in a position of power to raise team and group awareness on an organisational level as well as amongst subordinates.

Proposition 5b: Successful teams and groups need task- and relationship orientated management.

Proposition 5c: Intergovernmental organisations require managers who possess skills to integrate political, economical and social requirements.

\subsubsection{Employee - Co-worker Relationship}

The employee-co-worker relationship is characterised by a variety of factors which influence the individual's behaviour with regards to group and team work. Diversity is the most salient factor of influence. Ely \& Thomas (2001) suggest that diversity is more likely to yield positive outcomes when the organisational context views diversity as an opportunity for learning and integration. This is true for the United Nations Organisations under investigation where diversity manifests one of the major fundamentals. Diversity is seen as source of knowledge. Nonetheless, diversity as a major contextual aspect of group and team work triggers several implications. The most salient ones will be discussed in the following.

Overall, the structure of the United Nations Organisations creates an environment in which national diversity is not salient. This means that the work forces' ethical diversity is so high that a dominance of one nationality in daily work life is not possible. This phenomenon was reported by interviewees working in the locations in Geneva, Vienna and New York. However, people who work in the field, which means that they work in foreign countries outside the headquarters, reported that national diversity became salient.

One decisive barrier for group and team work which results from diversity is the influence of the use of a second language (which predominately is English) as a major means of communication. The proficiency of English determines the degree of mutual understanding among individuals. It is a source of misunderstandings, misinterpretations and in the worst case results in conflict. Hence, it is suggested to reinforce language trainings to reduce this source of misunderstanding. At the same time, it lies in the hands of management to create an environment which knows how to deal with the language barrier and how to overcome it.

Despite the fact that diversity is regarded as a source of enrichment, it is at the same time a trigger for the creation of faultlines. On one hand it is suggested to 
take advantage of the formation of subgroups as they may also be seen as knowledge creating source. On the other hand, flattened team and group structures might reduce the propensity to form subgroups and encourage acting as one entity.

Proposition 6: Diversity in its various facets is the major influence on the relationship between individuals.

Proposition 6a: The culture of intergovernmental organisations creates an environment in which diversity is not salient.

Proposition 6b: National and cultural diversity become especially salient when working outside of the headquarters.

Proposition 6c: Communication in a second language is a major reason for misunderstandings between individual group members.

Proposition 6d: Faultlines are a source of knowledge.

Proposition 6f: Flattened team structures reduce the formation of subgroups.

\subsubsection{Individual}

To understand an organisation it is vital to examine the individuals in the group and their interaction. Data analysis showed that (apart from the organisational structure) interviewees ascribe the success of group and team work to the individual.

In the course of data analysis it was revealed that the organisation tries to select individuals who share common characteristics with regards to their educational background and their international experience. Hence, staff of the United Nations Organisations can be characterised as being experienced in international and multicultural work and as being used to diversity at the work place.

The individual's motivation has been found to be a huge factor of influence on work. The feeling of contributing to something that has a positive impact on the whole society motivates individuals. This motivation is of relevance for cooperation and group work. Analysis suggests that most people who have lost this feeling over the years are more reluctant to engage in group work.

Lastly, the personality of each individual in the group affects group behaviour and performance. The unique characteristics of individuals determine whether or not two or more individuals are able to constructively work together or not. A fit of individuals was reported to be highly important. Both motivation and person- 
ality are factors which are not visible but become salient throughout the process of collective work.

Proposition 7: The individual and his/her personal background, personality and motivation are factors of influence on group and team work.

Proposition 7a: Internationally experienced staff are more proficient in dealing with diversity.

Proposition 7b: The personal characteristics of individuals have a stronger influence on group and team work than nationality.

Proposition 7c: In an intergovernmental organisation, group and team work as a source of individual motivation increases performance. 


\section{Discussion and Conclusions}

\subsection{Introduction}

This study aimed at developing an understanding of multicultural work in five Organisations of the United Nations guided by two main research questions:

- What kind of team and group work in five United Nations Organisations exists?

- Which factors are a trigger, a barrier, and/or an influence on team and group work in this context?

These research questions helped to examine three gaps in current research on multicultural team and group work.

First, whereas intense research on teams and groups in international companies has been conducted, this study focused on international organisations. The context of international organisations differs in many ways from international companies beginning with the legal base, the financial and operational agenda, the cultural impact and also with regards to human resource practices like selection of candidates, training, international placements, career management and remuneration. As a number of critics have noted (e.g. Rousseau \& Fried, 2001, Jackson et al., 2003) a rich description of the context is the key for valid interpretations of research.

The second gap that was addressed in this study focused on an evaluation of the types of teams and groups that can be found in international organisations. Management research has produced a plethora of studies on teams and groups in past decades. A clear definition of the underlying concepts was, however, found to be missing. This study not only evaluated research into teams and groups conducted between 1998 and 2007 but also accentuated the need for clear definitions of constructs as being essential for accurate research. Based on 50 interviews a typology of group work in the context of five Organisations of the United Nations was provided. Third, this study provided an integrative view of the reciprocal relationships between organisation, management, group and individual. By looking at the manner in which context affects these relationships, the triggers and barriers for team and group work could be explored. This way, the current study made an important step towards a more integrative view of organisational behaviour.

\subsection{Summary of research findings}

A qualitative research design was chosen to conduct this study. 25 semistructured interviews with 50 predominately Austrian staff members of five Organisations of the United Nations in three locations were the basis for this case 
study with embedded units of analysis (Yin, 1984). With the help of qualitative data analysis software, namely Atlas.ti Version 5, the interviews were analysed according to constant comparison method (Glaser \& Strauss, 1967). This method proved to be a valid tool to discover patterns and to make sense of a huge amount of interview data.

The results of data analysis and their contextualisation showed a strong influence of organisational structure on multicultural work. Moreover, the strong hierarchy and bureaucracy in the five Organisations of the United Nations that were investigated were found to be the largest barrier for team work. In fact, the structure of these Organisations with prevailing hierarchy and task specialisation conflicts with team work that is characterised by flat hierarchies. The novelty of this study is that data analysis strongly indicated the existence of group work and led to develop a typology comprising task forces, project groups, and collaboration. This typology emerged throughout the analysis of data and whilst discovering triggers and barriers for work in the context of international Organisations.

From a micro-level of analysis this study illuminated the reciprocal relationships between organisation, management, group and individual. By looking at these relationships, individual perceptions could be unfolded and an integrative model of multicultural work could be developed.

Overall, the results provided strong evidence that the hierarchical structure of the Organisations under investigation is the strongest barrier for team work. At the same time boundary breaking and boundary spanning activities on an organisational as well as on an individual level take place to overcome these barriers. Team work could be a remedy for institutional slack and as an individual source of motivation from each of the actor's point of view.

In the following the two research questions will be addressed in more detail and a summary of the findings will be presented accordingly.

\section{What kind of team and group work exists in United Nations Organisations?}

To start with it was reviewed how individuals construe team and/or group work in intergovernmental Organisations. Despite the fact that almost all interviewees initially used the word 'team' to define their work situation it quickly became clear that the underlying meaning did not refer to the term 'team' as per definition. Interviewees then described the position and tasks of their co-workers or superiors. Analysis further showed that around $60 \%$ of all interviewees' interpretations of teams and group work could be categorised around communication and coordination. Both elements were described to be essential to fulfil individual tasks. In the course of their narratives it became clear to respondents that some elements which are distinctive for team work were missing in their description of the work situation. The missing elements could be grouped around a lack of decision making power of individuals, a lack of collective performance 
measurement and most importantly, the prevalence of a very strong hierarchy and bureaucracy.

In a next step the explanations of the respondents with regard to team and group work were compared with theoretical concepts of team and group work in academic literature. The definitions chosen were provided by Katzenbach \& Smith (1993) and seemed compelling because of their simple but comprehensive description.

Definition of the term "team":

A team is a number of 2 or more people who are committed to a common goal which is specific and different from each team member's individual goals. Team members work collectively to reach this goal. They share leadership roles and follow a collective decision making process. Furthermore, the solution to problems is found in the course of collective discussion. The outcomes of the team work are subject to collective performance measurement.

Definition of the term "group":

A group is a number of 2 or more people who are committed to a common goal which is similar to the broader organisational goals and each group member's individual goal. Group members work individually to reach their goals. The leadership role is centralised at one person who is also responsible for the decision making process and who delegates individual tasks. Finally, the outcomes of group work are subject to individual performance measurement.

Based on these clear definitions the following conclusions could be drawn: the hierarchical structure implies task specialisation and as a result, individuals work on individual tasks. Hand in hand with specialisation comes a differentiation by groups of employees comprising different degrees of authority and power. Tasks are delegated in a top down approach and performance is measured individually. All of these characteristics indicate that teams can not exist in the context of the five Organisations of the United Nations under investigation. A comparison of the interview data with the definitions referred to the following typology of group work including task forces, project groups and collaboration.

A task force operates temporarily in case of non-routine events or emergency events. Once the problem is solved, a task force usually will be dissolved. Conventional hierarchies marginally apply or do not apply to task forces at all.

A project group is established for the duration of a specific project. Project groups typically have an assigned group leader and tasks are assigned according to specialisation.

Collaboration takes place on a regular basis and is not timely limited. The formal hierarchy applies and tasks are executed based on task specialisation. There is no necessary common goal among collaborating individuals.

The characteristics of each of the types are briefly summarised in the table below. 
Table 8: Typology

\begin{tabular}{|l|l|l|l|}
\hline Type & Duration & Task & Hierarchy \\
\hline Task force & temporary & non-routine & suspended \\
\hline Project group & specified period & specified & $\begin{array}{l}\text { Group leaders and } \\
\text { members are equal }\end{array}$ \\
\hline Collaboration & undefined & specialisation & effective \\
\hline
\end{tabular}

Source: author

This typology covers the explanations of the 50 interviewed staff members in five Organisations of the United Nations, namely IAEA, UNIDO, UNDP, UNOPS, and WIPO.

\section{Which factors are a trigger, a barrier and/or a factor of influence on team and group work in this context?}

Deriving from interview data analysis the main factors which are a barrier, a trigger and a factor of influence on team and group work could be classified into three categories. These categories are: the organisation, relationships, and individuals. Each of the categories will be summarised briefly in the following and labelled as trigger, barrier or as a factor of influence.

\section{Organisation}

The organisation and its structure was found to be a strong barrier for team work and an equally strong factor of influence on group work and work in task forces, project groups and collaboration. The structure of the United Nations Organisations under investigation is depicted by hierarchy and bureaucracy. Hierarchy is the formal structure which shows different levels of power, responsibility and authority. It also identifies accountability. The existence of a strong hierarchy was emphasised by $95 \%$ of all interviewees. Bureaucracy expressed by rules and regulations and perceived as an instrument of power and control dominated by the top level of a hierarchy, was described as another strong factor of influence on group work and collaboration. According to more than $50 \%$ of all interviewees, priority has to be put on understanding and following rules and regulations.

The personnel system or Human Resource Management of the United Nations Organisations can be considered as a factor of influence on group work and is also determined by the structure of the organisation. Recruiting processes, contracts, career development, and termination of contracts are equally influenced by hierarchy and bureaucracy. The processes were described as being cumbersome and very lengthy. Recruiting for example is affected by political motives. A plethora of different kinds of contractual arrangements exists. One distinctive contractual feature is the so called job rotation principle which is applicable for predominately high ranking jobs. Rotation is motivated by political issues but 
also a strategy to spread knowledge in a learning organisation. Whilst a change in leadership every three years can be seen as a source for fresh ideas, it also hinders stability because of changing practices and additional rules and regulations.

Career development is also restricted by hierarchical structures and influenced by political actions. Performance measurement is practised on an individual level and as a consequence, individuals try to shed light on individual efforts before putting energy and resources in group work. Moreover, this style of performance measurement does not induce group spirit and cohesion.

Despite strong hierarchies and strict bureaucracy, the Organisation as well as individuals are actively breaking down these boundaries and spanning new ones. On an organisational level boundary spanning activities emphasise knowledge transfer beyond hierarchies and more direct communication neglecting hierarchies. The transfer of tacit and explicit knowledge as a source of organisational learning and building up of organisational memory is regarded as being vital for a well functioning organisation. On an individual level boundary spanning activities take place for several reasons: to reduce a deficit in information, to work more efficient beyond hierarchy, or to gather information faster. A parallel system and network to the formal hierarchy evolves due to boundary activities and helps individuals to fulfil their tasks more efficiently.

Lastly, the culture of the organisation has also been described as being dominated by hierarchy. Symbols like the number of windows in an office or the size of an office manifest organisational culture. Pattern of communication in the form of jargon and terminology as well as the expression of power by hierarchy depict the organisational culture on a visible level. Held values, beliefs, assumptions and attitudes that underlie behaviour characterise the invisible level of organisational culture which was made visible by looking at relationships between individuals.

\section{Relationships}

Two types of relationships were analysed as being crucial when it comes to explaining triggers and barriers for task forces, group work and collaboration. First, the relationship between employee and management, and second the relationship between employee and co-worker. Each of them will be summarised briefly in the following.

\section{Employee - Management Relationship}

Management as perceived by individuals is the major link between employees and the organisation. Consequently, individuals' trust and confidence in management is crucial for successful performance. This trust was explained to be spoiled by politically appointed managers and hampered because of frequent 
rotation of managers. Interviewees believe that a manager who builds up trust, and motivates and empowers individuals is the key to successful group work. The manager is also seen in a position of power to set the stage for team work and to trigger group spirit.

\section{Employee - Co-worker relationship}

The relationship between employees and co-workers is depicted by diversity: national and cultural diversity, stereotypes, gender differences, language and resulting faultlines, respectively subgroups. The facets of diversity mentioned by interviewees will be summarised below.

By its very nature, the United Nations Organisation is a multicultural work place and subsequently national and cultural diversity are facts of life of daily work. Interviewees see each other as part of a community in which nationality should not matter - just like it is stipulated in the UN Charter. In the course of daily work life distinctive characteristics of national culture become salient and influence work. Data analysis showed that the salience of diversity differs depending on the work environment. Interviewees working in the headquarters elaborated on cultural differences such as religion and cultural traditions or food. These differences were described as being interesting and as factors which make work in United Nations Organisations special and exciting. Staff working in the field reported differently. Stereotypes and cultural differences were perceived as a barrier for effective work. Depending on the country in which interviewees gained their field experience, they reported of stronger hierarchies and lines of authority as compared to the headquarters, less decision making power for individuals, different feeling for time and little effort of individuals to take responsibility. The interviewees reported two possible strategies to cope with these differences: maintenance of their own cultural identity or building up of relationships with other groups. Both acculturation strategies were equally implied among interviewees.

Gender issues such as under representation of females in top level positions and an over representation of women in administrative jobs was reported despite the fact that the United Nations staffing policies aim at an equal participation and representation of gender across all hierarchies.

Lastly, language diversity was noted to have a considerable impact on work. English as the major language of communication can be a barrier for group work from the point of view of non-native English speakers. The individuals' proficiency of English determines whether meetings are dominated by native speakers or not and whether comments of non-native speakers are regarded as competent or not.

Austrian interviewees see language as a barrier for communication and exchange of information and as a result as a factor of influence on group work. To overcome this barrier, interviewees reported of two possibilities: the use of tech- 
nical language as an element of professional culture, and the use of simple language to ensure that the content of communication becomes clear.

All of the facets of diversity described above lead to the emergence of faultlines, respectively subgroups. Faultlines as defined by Lau \& Murnighan (1998) are 'hypothetical dividing lines that may split a group into subgroups based on one or more attributes.' Data analysis strongly supported the existence of subgroups because of gender, race, age and functional diversity. A single attribute can be a trigger for the creation of a faultline. In a group context, these dividing lines cause individuals to see other group members either as part of their in-group or as part of an out-group. Research suggests (e.g. Early \& Mosakowski, 2000) that groups with maximum diversity experience less dividing lines and the propensity of an emergence of in- and out-groups is smaller. The United Nations Organisation as a hierarchical organisation especially faces faultlines because of strict lines of power and decision.

A moderating factor of influence on all of the relationships is the individual characterised by personal background, personality and motivation. The personal background of individuals in this study comprises experience in an international context such as growing up in multicultural families and foreign countries. It also comprises education and/ or work experience in foreign countries.

The personality of each individual is a relevant issue when it comes to group work. Empirical analysis showed that personality may be supportive for group work and collaboration while it may also be a barrier.

Individual motivation derives from intrinsic motives such as intellectual stimulation or trust that work has an impact while extrinsic motivation is induced by remuneration and benefits.

A conceptual model of group work in five Organisations of the United Nations Organisation graphically illustrates the interplay of all the categories described in the section above (Figure 11).

\subsection{Managerial implications}

The following section develops practical advice for management in the United Nations Organisations. Based on the empirical findings of this study, which showed that management is the crucial link between the organisation and the individuals, the managerial implications were developed around two main questions:

- How to manage hierarchy and bureaucracy?

- How to manage relationships?

This section will conclude in managerial guidelines derived from empirical analysis and based on the propositions developed. The guidelines aim at devel- 
oping how group and team work in a hierarchical and diverse intergovernmental organisation can be put into practise.

\section{How to manage hierarchy and bureaucracy?}

Working for a United Nations Organisation can be a rewarding and fulfilling job given the opportunities the United Nations System offers all over the globe. The international context characterised by diversity in all aspects of work life, and organisational goals that really aim at changing the world seem compelling. When working for the United Nations one must not forget, however, that this organisation has become very large over the past 61 years and as a consequence, hierarchy and bureaucracy became very strong. The latter are a fact of daily work life in a world organisation such as the United Nations and mainly aim at assuring accountability and clear lines of responsibility and decision making power. At the same time hierarchy and bureaucracy were found to be the main sources of dissatisfaction. Both were identified as a barrier for team work and a major influence on group work and collaboration. $90 \%$ of interviewees reported perceiving hierarchy and too many rules and regulations as a nuisance in fulfilling their individual tasks. Without any doubt, hierarchy and bureaucracy will always be a major characteristic of huge organisations. However, a systematic questioning of some of the rules and regulations might show that over the years incremental rules and regulations have been introduced without being necessary. Management can be seen as central and in a position to reduce rules and regulations to the necessary minimum within the scope of a manager's responsibility. Furthermore, management is in a position of power to create a work environment beyond rules and regulations. Managers may share information equally among group members and introduce a culture of discussion and mutual exchange. By involving individual staff in the decision making process and by valuing individual's opinions and suggestions, a manager not only shares some of his/her responsibilities with the group but also increases individual motivation and as a result, performance.

A real remedy for strong hierarchies and institutional slack is the implementation of teams comprising individuals who work collectively to reach a common goal, who share leadership roles and collectively make decisions. Subsequently, they should be subject to collective performance measurement.

Management is in a position to raise team awareness by offering training to individual staff and by actively breaking down institutional boundaries. Interviewees in management positions have reported that all of this is possible and depends on the individual manager's possibilities and effort.

\section{How to manage relationships?}

Relationships in the United Nations Organisations are depicted by diversity in various facets. National and cultural diversity most strongly influence relation- 
ships. Management has to be aware of the fact that diversity is not only an asset but also a barrier. Therefore, a manager of a diverse group has to be sensitive towards different norms of behaviour and attitudes. It is suggested not to underestimate the effect of the emergence of subgroups. Therefore, management is advised to establish group norms by clearly defining communication styles, discussion culture and mutual considerateness. By offering intercultural training to individual group members, cultural awareness and mutual understanding can be raised and trained. As a consequence, a better understanding of each other would lead to improved communication and task fulfilment and to increased motivation. Moreover, knowledge and information would not be seen as a means of power but as a means to build up collective and shared knowledge.

When it comes to managing employee-co-worker relationships, management has to be aware of personality traits of individuals. For instance, some individuals are more extroverted or more assertive as compared to others. Management should be able to assess personality traits and to assign roles within a group or team according to the decisive characteristics of each personality. Together with collectively established team and group norms, a fit of individuals in team and group roles can be seen as a pathway to effective performance by satisfied and motivated individuals.

Five managerial implications to make group and team work in a hierarchical and bureaucratic structure work: creating a hybrid culture

The clue to successful group work and a future implementation of team work structures in the five UN organisations lies in the strategic use of hierarchy, bureaucracy and in assigning the right management. The major challenge is to design a culture in which both hierarchy and team work can co-exist without turning the established organisational structure upside down, namely a hybrid culture.

The following section lists and subsequently describes five normative practices that lead to a hybrid culture.

\section{Analyse hierarchical (boundary activities) and bureaucratic (HRM) processes.}

2. Reduce processes to the minimum needed which still assures authority, accountability and credibility but gives the individual in charge responsibility.

3. Span boundaries to enable team work.

4. Design HRM practices supporting a hybrid culture

5. Ensure the requisite skills of managers. 
The structures which hierarchy offers should be used for routine tasks which have to be executed on a regular basis and according to the defined rules and regulations. By using hierarchy and established bureaucracy to fulfil these tasks, their smooth and fast accomplishment can be guaranteed. However, it is advised to question existing hierarchies and bureaucracies in order to reduce redundancy and to guarantee clear lines of authority. This process is advised to be done in the light of boundary spanning activities and with regards to Human Resource Practices. Both will be explained as follows:

For non-routine tasks it is suggested to span boundaries by opening the hierarchy to team structures. Team structures with dedicated roles and responsibilities do not necessarily conflict with the organisational premises of accountability and credibility. Yet, team structures may at the same time increase creativity, motivation, and subsequently performance. Research has shown that performance improves when the roles of individual team members are clearly defined and well understood (Gratton \& Erickson, 2007). Hence, individuals feel that they can work independently within the hierarchical structure while they have at the same time the possibility to cooperate across boundaries.

A hybrid culture needs Human Resource practises which are team orientated rather than individually orientated. These practices provide collective decision making, shared leadership roles, collective discussion and solution of problems as well as collective performance measurement. The practices also foster training in skill related and collaborative behaviour.

Human Resource Management practises should emphasise a selection of managers who possess the skills and abilities to manage teams. Therefore, career development should also consider skills which are especially important when it comes to lead and guide teams. Hence, managers should possess of political, economic and social skills. Effective teams and groups need managers who are both task- and relationship orientated. Moreover, these managers need to be able to switch between the two orientations during team and group work in order not to hinder the performance of such teams in the long run. Managers who possess these skills may become designers of successful teams and groups. By demonstrating collaborative behaviour themselves, managers act as a role model for their subordinates and employees. By mentoring and coaching, managers also create a collaborating atmosphere which will positively enhance group and team work on an individual level.

The five implications are summarised in the figure 12 below: 
Figure 12: Five managerial implications

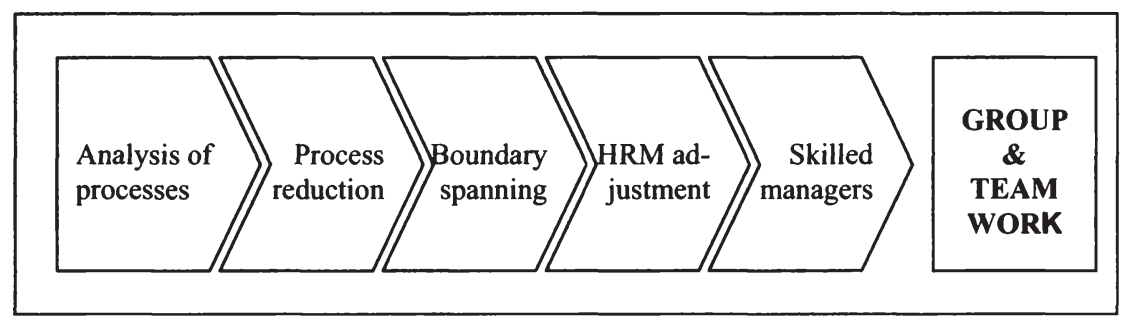

Source: data analysis 


\subsection{Limitations and directions for future research}

The findings of this study should be viewed in light of several limitations. These limitations will be addressed in the following and suggestions for future research will be provided.

Firstly, the generalisability of the results to the whole UN system is limited by sample restrictions. This study aggregated an Austrian perspective on multicultural work in the United Nations Organisations. The reason for the restriction to an Austrian and German speaking sample originates in a trade off. To avoid a cross-cultural bias when conducting interviews in a foreign language, the decision was made to only interview native German speakers from Austria and from Germany.

Therefore, an interesting avenue for future research would be to conduct interviews in different languages with different nationalities and cultures.

Secondly, the study is limited to five individual Organisations of the United Nations System. This reason for this restriction was twofold: on the one hand, one single researcher can not possibly cover the huge body of the United Nations System because of time and resource limitations. A selection had to be made and it paid attention to covering different types of units of the United Nations System, namely agencies, programmes and organisations. On the other hand, those units were chosen were the majority of German speaking staff could be identified.

Future research should aim at investigating other units to get a broader view of the organisational context.

Thirdly, this study is completely qualitative. The data collection method using semi-structured interviews is qualitative, the data analysis using constant comparison method is qualitative and the method being a case study is qualitative. Qualitative research is criticised as being biased by the researcher. Because of smaller sample size it is limited to a specific context which therefore has to be richly described. A quantitative research design may help to make results more generalisable in future research.

Finally, the model presented covers a small slice of the vast domain of multicultural work and intergovernmental organisations. A longitudinal study comprising researchers from different research fields would help to widen the scope and broaden the understanding of multicultural work in this specific context. 


\section{Bibliography}

Adler, N. J. 2002. International Dimensions of Organizational Behaviour. Cincinnati, Ohio.

Alderfer, C. P. 1977. Group and intergroup relations. In Improving the Quality of Work Life, ed. JR Hackman, JL Suttle, : Pallisades, CA: Goodyear.

Alderfer, C. P., \& Thomas, D. A. 1988. The signifcance of race and ethnicity for organizational behavior. . In C. L. Cooper, \& I. Roberston (Eds.), International review of industrial and organizational psychology. New York: Wiley.

Allison, C. W. 1984. Bureaucratic Personality and Organisation Structure: Gower Publishing House.

Alper, S., Tjosvold, D., \& Law, K. S. 2000. Conflict management, efficacy, and performance in organizational teams Personnel Psychology, 53: 625642.

Ambos, B., \& Schlegelmilch, B. 2004. The use of international R\&D teams: an empirical investigation of selcted contingency factors. Journal of World Business, 39: 37-48.

Ambrosini, V., Bowman, C., \& Burton-Taylor, S. 2007. Inter-team coordination activities as a source of customer satisfaction. Human Relations, 60(1): 59-98.

Ancona, D., \& Caldwell, D. F. 1988. Beyond task and maintenance: defining external functions in groups. Groups and Organisation Studies, 13: 468494.

Annan, K. 2006. Investing in the United Nations: for a stronger Organisation worldwide, Report of the Secreatary-General.

Armstrong-Stassen, M., Wagar, T. H., \& Cattaneo, R. J. 2004. Work-group membership (in)stability and survivors' reactions to organizational downsizing. Journal of Applied Social Psychology, 34: 2023-2044.

Ayoko, O. B., \& Härtel, C. E. J. 2003. The role of space as both a conflict trigger and a conflict control mechanism in culturally heterogeneous workgroups. Applied Psychology: An international review, 52(3): 383-412.

Babbie, E. 1992. The Practice of Social Research,9th edition. Stamford, CT: Wadsworth/Thomson Learning.

Barinaga, E. 2007. Cultural diversity' at work: 'National culture' as a discourse organizing an international project group. Human Relations, 60(2): 315340.

Barrick, M. R., Stewart, G. L., Neubert, M. J., \& Mount, M. K. 1998. Relating Member Ability and Personality to Work-Team Processes and Team Effectiveness. Journal of Applied Psychology, 83: 377-391.

Beetham, D. 1996. Bureaucracy (2 ed.). Buckingham: Open University Press. 
Beigbeder, Y. 1987. Management Problems in United Nations Organisations. Reform or Decline? London: Frances Pinter.

Beigbeder, Y. 1997. The internal management of the United Nations organizations - The long quest for reform. NY: St. Martin's Press.

Berry, J. W. 1984. Cultural Relations in Plural Societies: Alternatives to Segregation and their Sociopsychological Implications. In: Miller, N. and Brewer, M.B. (eds) Groups in Contact: the Psychology of Desegregation: 11-27. Orlando, FL: Academic Press.

Bettenhausen, K. L. 1991. Five Years of Groups Research: What We Have Learned and What Needs to be Addressed. Journal of Management, 17: $345-381$.

Bewley, T. 2002. Interviews as a valid empirical tool in economics. The Journal of Socio-Economics, 31: 343-535.

Bird, A., \& Stevens, M. J. 2003. Toward an emergent global culture and the effects of globalization on obsolescing national cultures. Journal of International Management(9): 395-407.

Bishop, J. W., \& Scott, K. D. 2000. An examination of organizational and team commitment in a self-directed team environment. Journal of Applied Psychology, 85: 439- 450.

Bishop, J. W., Scott, K.D., \& Burroughs, S.M. 2000. Support, commitment, and employee outcomes in a team environment. Journal of Management, 26: 1113-1132.

Boeije, H. 2002. A purposeful approach to the constant comparative method in the analysis of qualitative interviews. Quality and Quantity, 36: 391-409.

Bortz, J., \& Döring, N. 2002. Forschungsmethoden und Evaluation für $\mathrm{Hu}$ man- und Sozialwissenschafter, 3. Ed., Berlin.

Bowers, C. A., Pharmer, J. A., \& Salas, E. 2000. When member homogeneity is needed in work teams - a meta analysis. Small Group Research, 31: 305327.

Bozemann, B. 2000. Bureaucracy and Red Tape. N.J.: Prentice-Hall.

Bozemann, B., \& Loveless, S. 1987. Sector Context and Performance: A Comparison of Industrial and Government Research Units. Administration and Society, 19(2): 197-235.

Brenner, O. C., Tomkiewicz, J., \& Schein, V. E. 1989. The relationship between sex role stereotypes and requisite management characteristics revisited. Academy of Management Journal, 32: 662-669.

Brewer, M. B. 1979. In-group bias in the minimal intergroup situation: A cognitive-motivational analysis. Psychological Bulletin, 86: 307-324.

Brief, A. P., Umphress, E. E., Dietz, J., Burrows, J. W., Butz, R. M., \& Scholten, L. 2005. Community matters: realistic group conflict theory and the impact of diversity. Academy of Management Journal, 48(5): 830-844. 
Bunderson, J. S. 2003. Recognizing and utilizing expertise in work groups: A status characteristics perspective. Administrative Science Quarterly, 48: 557-591.

Burke, C. S., Stagl, K. C., Salas, E., Pierce, L., \& Kendall, D. 2006. Understanding Team Adaptation: A Conceptual Analysis and Model. Journal of Applied Psychology, 91(6): 1189-1207.

Caligiuri, P. M. 2000. Selecting expatriates for personality characteristics: a moderating effect of personality on the relationship between host national contact and cross-cultural adjustment. Management International Review, 40(1): 61-80.

Cannon, M. D., \& Edmondson, A. C. 2001. Confronting failure: Antecedents and consequences of shared beliefs about failure in organizational work groups. Journal of Organizational Behavior, 22: 161-177.

Cappelli, P., \& Sherer, P. D. 1991. The missing level of context in OB: the need for a mesolevel approach. Research in Organisational Behaviour, 13: 55-110.

Carless, S. A., \& De Paola, C. 2000. The measure of cohesion in work teams. Small Group Research, 31: 71-88.

Chang, S., \& Tharenou, P. 2004. Competencies needed for managing a multicultural workgroup. Asia Pacific Journal of Human Resources, 42(1): 5774.

Chansler, P. A., Swamidass, P. M., \& Cammann, C. 2003. Self-managing work teams. An empirical study of group cohesiveness in "natural work groups" at a Harley-Davidson motor company plant. Small Group Research, 34: 101-120.

Charter. Charter of the United Nations.

Chatman, J. A., \& O'Reilly, C. A. 2004. Asymmetric reactions to work group sex diversity among men and women. Academy of Management Journal, 47: 193-208.

Chattopadhyay, P. 1999. Beyond direct and symmetrical effects: the influence of demographic dissimilarity on organizational citizenship behavior Academy of Management Journal, 42(3): 273-287.

Chattopadhyay, P., Tluchowska, M., \& George, E. 2004. Identifying the ingroup: a closer look at the influence of demographic dissimilarity on employee social identity. Academy of Management Review, 29: 180-202.

Chen, G., Kirkman, B. L., Kanfer, R., Allen, D., \& Rosen, B. 2007a. A Multilevel Study of Leadership, Empowerment, and Performance in Teams. Journal of Applied Psychology, 92(2): 331-346.

Chen, S., Geluykens, R., \& Choi, C. J. 2006. The importance of language in global teams: a linguistic perspective. Management International Review, 46(6): 679-695.

Chen, Z., Lam, W., \& Zhong, J. A. 2007b. Leader-Member Exchange and Member Performance: A New Look at Individual-Level Negative Feed- 
back-Seeking Behavior and Team-Level Empowerment Climate. Journal of Applied Psychology, 92(1): 202-212.

Chevrier, S. 2003. Cross-cultural management in multinational project groups. Journal of World Business, 38: 141-149.

Child, J. 2005. Organization - Contemporary Principles and Practice: Blackwell.

Choi, J. N. 2002. External activities and team effectiveness: Review and theoretical development. Small Group Research, 33: 181-208.

Cohen, S. G., \& Bailey, D. E. 1997. What Makes Teams Work: Group Effectiveness Research From the Shop Floor to the Executive Suite. Journal of Management, 23: 239 - 290.

Colquitt, J. A., Noe, R. A., \& Jackson, C. L. 2002. Justice in teams: Antecedents and consequences of procedural justice climate. Personnel Psychology, 55: 109.

Corbin, J., \& Strauss, A. 1990. Grounded Theory Research: Procedures, Canons, and Evaluative Criteria. Qualitative Sociology, 13(1).

Corderly, J. 2003. Team work. In Holman D, Wall TD, Clegg CW, Sparrow P, Howard A (Eds.). The new workplace: A guide to the human impact.

Costa, P. T., \& McCrae, R. R. 1992. Four ways five factors are basic. Personality and Individual Differences, 13: 653-665.

Creswell, J. W. 1994. Research Design: Qualitative \& Quantitative Approaches. Thousand Oaks: Sage.

Cross, R. L., Yan, A., \& Louis, M. R. 2000. Boundary Activities in "Boundaryless" Organizations: A Case Study of a Transformation to a Team-Based Structure. Human Relations, 53: 841-868.

Davenport, T. H., \& Klahr, P. 1998. Managing customer support knowledge. California Management Review, 40: 195-208.

De Dreu, C. K. W. 2006. When Too Little or Too Much Hurts: Evidence for a Curvilinear Relationship Between Task Conflict and Innovation in Teams. Journal of Management, 32(1): 83-107.

De Dreu, C. K. W., \& Weingart, L.R. 2003. Task and relationship conflict, team performance, and team member satisfaction: a meta analysis. Journal of Applied Psychology, 88: 741-749.

De Dreu, C. K. W., \& West, M. A. 2001. Minority dissent and team innovation: The importance of participation in decision making. Journal of Applied Psychology, 86: 1191-1201.

Denison, D. R., Hart, S.L., \& Kahn, J.A. 1996. From chimneys to cross functional teams: Developing and validating a diagnostic model. Academy of Management Journal, 39(4): 1005-1023.

Driskell, J. E., Goodwin, G. F., Salas, E., \& O'Shea, P. G. 2006. What Makes a Good Team Player? Personality and Team Effectiveness. Group Dynamics, 10(4): 249-271. 
Dyer, W. G. 1987. Team building: Issues and alternatives (2nd ed.) Reading, MA: Addison-Wesely.

Earley, P. C., \& Mosakowski, E. 2000. Creating hybrid team cultures: an empirical test of transnational team functioning. Academy of Management Journal, 43(1): 26-49.

Eby, L. T., Adams, D. M., Russell, J. E. A., \& Gaby, S. H. 2000. Perceptions of organizational readiness for change: Factors related to employees' reactions to the implementation of team-based selling. Human Relations, 53: 419-442.

Eisenhardt, K. M. 1989. Building Theories from Case Study Research. Academy of Management Review, 14(4): 532-550.

Ellemers, N., de Gilder, D., \& Van den Heuvel, H. 1998. Career-Oriented Versus Team-Oriented Commitment and Behavior at Work. Journal of Applied Psychology, 83: 717 - 730.

Elloy, D. F., Terpening, W., \& Kohls, J. 2001. Causal model of burnout among self-managed work teams' members Journal of Psychology, 135: 321334.

Elron, E., Halevy, N., Ari, E. B., \& Shamir, B. 2003. Cooperation and coordination across cultures in the peacekeeping forces: Individual and organisational integration mechanisms. In T. W. Britt, \& A. B. Adler (Eds.), The Psychology of the Peacekeeper: Lessons from the Field. Westport.

Ely, R. J. 2004. A field study of group diversity, participation in diversity education programs, and performance. Journal of Organizational Behavior, 25: 755-780.

Ely, R. J., \& Thomas, D. A. 2001. Cultural diversity at work: the effects of diversity perspectives on work group processes and outcomes. Administrative Science Quarterly, 46: 229-273.

English, A., Griffith, R. L., \& Steelman, L. A. 2004. Team performance: The effect of team conscientiousness and task type Small Group Research, 35: 643- 665 .

Feely, A. J., \& Harzing, A. W. 2003. Language management in multinational companies. Cross Cultural Management, 10(2): 37-52.

Feldman, M. S. 2005. Management and Public Management. Academy of Management Journal, 48(5): 958-960.

Fernandez, F. 2005. Globalization and Human Resource Management Adapting Successful UN Practices for the Private and Public Sectors. New York: HNB Publishing.

Fink, G., \& Holden, N. 2007. Resistance by host country staff in international ventures - a theoretical sketch, VHB-Kommission Internationales Management. Vienna.

Fink, G., Kölling, M., \& Neyer, A. K. 2004. Research on intercultural management interaction: the cultural standard method. Unpublished Paper, Wirtschaftsuniversität Wien. 
Fink, G., \& Meierewert, S. 2004. Issues of time in international, intercultural management: East and Central Europe in the perspective of Austrian managers. Journal for East European Management Studies, 9(1).

Flick, U., Karldorff, v. E., \& Steineke, I. 2000. Qualitative Forschung - ein Handbuch. Hamburg.

Flynn, F. J., Chatman, J. A., \& Spataro, S. E. 2001. Getting to know you: the influence of personality on impressions and performance of demographically different people in organizations. Administrative Science Quarterly, 46(3): 414-446.

Forsyth, D. 1999. Group dynamics (3rd ed.), Belmont, CA: Wadsworth of modern working practices (pp. 95-114). Hoboken, NJ: Wiley.

Francis, D., \& Young, D. 1979. Improving work groups: A practical manual for team building. San Diego: University Associates.

Garcia-Prieto, P., Bellard, E., \& Schneider, S. C. 2003. Experiencing diversity, conflict and emotions in teams. Applied Psychology: An international review, 52(3): 413-440.

Gareis, S. B., \& Varwick, J. 2005. The United Nations - An Introduction (3 ed.): Palgrave MacMillan.

Geertz, C. 1973. Interpretation of Culture. New York: Basic Books.

Gephart, R. 2004. Qualitative Research and the Academy of Management Journal. Academy of Management Journal, 47(4): 454-462.

Gersick, C. 1988. Time and transition in work teams: Toward a new model of group development. Academy of Management Journal, 31: 9-41.

Gibson, C. B., \& Vermeulen, F. 2003. A healthy divide: subgroups as a stimulus for team learning behaviour. Administrative Science Quarterly, 48: 202239.

Gibson, C. B., \& Zellmer-Bruhn, M. E. 2001. Metaphors and meaning: an intercultural analysis of the concept of teamwork. Administrative Science Quarterly, 46(2): 274-300.

Gilson, L. L., Mathieu, J. E., Shalley, C. E., \& Ruddy, T. M. 2005. Creativity and standardization: Complementary or conflicting drivers of team effectiveness. Academy of Management Journal, 48: 521-531.

Gilson, L. L., \& Shalley, C. E. 2004. A little creativity goes a long way: An examination of teams' engagement in creative processes. Journal of Management, 30: 453- 470 .

Glaser, B., \& Strauss, A. 1967. The Discovery of Grounded Theory. Strategies for Qualitative Research. Aldine Publishing Company, Chicago.

Goodall, K., \& Roberts, J. 2003. Only connect: teamwork in the multinational. Journal of World Business 38: 150-164.

Gortner, H. F., Nichols, K. L., \& Ball, C. 2007. Organization Theory. A public and nonprofit perspective ( $3 \mathrm{rd}$ ed.). Belmont, CA: Thomson Wadsworth.

Gratton, L., \& Erickson, T. J. 2007. Ways to build collaborative teams. Harvard Business Review, November 2007: 100-111. 
Guzzo, R. A., \& Dickson, M. W. 1996. Teams in organizations-recent research on performance and effectiveness. Annual Review of Psychology, 47: 307-338.

Guzzo, R. A., \& Shea, G. P. 1992. Group performance and intergroup relations in organizations. In Handbook of Industrial and Organizational Psychology, ed. MD Dunnette, LM Hough, 3:269-313, Palo Alto, CA: Consult. Psychol. Press 2nd ed.

Hackman, J. R. 1983. A normative model of work team effectiveness. Technical report no. 2, School of Organization and Management, Yale University, New Haven, CT.

Hackman, J. R. 1987. The design of work teams. In Handbook of Organizational Behavior. Ed. JW Lorsch, pp. 315-342. Englewood Cliffs, NJ: Prentice-Hall.

Hare, P. A. 1992. Groups, Teams, and Social Interaction. Theories and Application Praeger.

Harrison, D. A., Price, K. H., \& Bell, M. P. 1998. Beyond relational demography: time and the effects of surface- and deep-level diversity on work group cohesion. Academy of Management Journal, 41: 96-107.

Henderson, J. K. 2005. Language Diversity in International Management Teams. Int.Studies of Management and Organisations, 35(1): 66-82.

Hermanowicz, J. 2002. The great Interview: 25 Strategies for studying people in bed. Qualitative Sociology, 25(4): 479-499.

Hirschfeld, R. R., Jordan, M. H., Feild, H. S., Giles, W. F., \& Armenakis, A. A. 2006. Becoming Team Players: Team Members' Mastery of Teamwork Knowledge as a Predictor of Team Task Proficiency and Observed Teamwork Effectiveness. Journal of Applied Psychology, 91(2): 467474.

Hobmann, E. V., Bordia, P., \& Gallois, C. 2003. Consequences of feeling dissimilar from others in a work team. Journal of Business and Psychology, 17(3).

Hoegl, M., \& Parboteeah, K. P. 2003. Goal Setting And Team Performance In Innovative Projects: On the Moderating Role of Teamwork. Small Group Research, 34(1): 3-19.

Hofstede, G. 1997. Cultures and Organizations: Software of the Mind. New York: McGraw-Hill.

Hofstede, G. 2001. Cultures' Consequences (2nd ed.). Thousand Oaks, CA: Sage.

Hofstede, G., \& Fink, G. 2007. Culture: organisations, personalities and nations. Gerhard Fink interviews Geert Hofstede. European Journal of International Management, 1(1/2): 14-22.

Holden, N. 1987. The treatment of language and linguistic issues in the current English-language management literature. Multilingua, 6(3): 233-246. 
Holden, N. 2002. Cross cultural management: a knowledge management perspective. London: Financial Times/Prentice Hall.

House, R., Hanges, P., Javidan, M., Dorfman, P., \& Gupta, V. 2004. Culture, Leadership and Organisations: the GLOBE Study of 62 Countries. Thousand Oaks, CA: Sage Publ.

Husemann, H. 1993. Doktor Seltsam: Oder wie ich lernte, das Stereotyp zu lieben. In J. P. Timm, \& H. J. Vollmer (Eds.), Kontroversen in der Fremdsprachenforschung. Bochum.

Hussey, J., \& Hussey, R. 1997. Business Research: A Practical Guide for undergraduate and postgraduate Students. Wiltshire, UK: McMillan.

Jackson, S. E. 1992. Team composition in organizational settings: issues in managing an increasingly divers work force. In: Group Process and Productivity, ed. S. Worchel, W. Wood, JA. Simpson, pp 136-180. Newbury Park, CA:Sage.

Jackson, S. E., Joshi, A., \& Erhardt, N. L. 2003. Recent research on team and organizational diversity: SWOT analysis and implications. Journal of Management, 29: 801-830.

Jackson, S. E., Joshi, A., \& Erhardt, N. L. 2004. Diversity in social context: a multi-attribute, multilevel analysis of team diversity and sales performance. Journal of Organizational Behavior, 25: 675-702.

Jehn, K. A., \& Bezrukova, K. 2004. A field study of group diversity, workgroup context, and performance. Journal of Organizational Behavior, 25: 703729.

Jehn, K. A., Northcraft, G. B., \& Neale, M. A. 1999. Why differences make a difference: a field study of diversity, conflict and performance of workgroups. Administrative Science Quarterly, 44(4): 741-763.

Jeménez, A. M. R., Górinak, J., Kosic, A., Kiss, P., \& Kandulla, M. 2004. European and National Identities in EU's Old and New Member States: Ethnic, Civic, Instrumental and Symbolic Components., Europen Integration online Papers (EIoP), Vol. 8: http://eiop.or.at/eiop/texte/2004-2011 a.htm.

Jiménez, A. M. R., Górinak, J., Kosic, A., Kiss, P., \& Kandulla, M. 2004. European and National Identities in EU's Old and New Member States: Ethnic, Civic, Instrumental and Symbolic Components., Europen Integration online Papers (EIoP), Vol. 8: http://eiop.or.at/eiop/texte/2004-2011 a.htm.

Joshi, A. 2006. The influence of organizational demography on the external networking behaviour of teams. Academy of Management Review, 31(3): 583-595.

Joshi, A., Labianca, G., \& Caligiuri, P. M. 2002. Getting along long distance: understanding conflict in a multinational team through network analysis. Journal of World Business, 37: 277-284.

Joshi, A., \& Lazarova, M. 2005. Do global teams need global leaders? Identifying leadership competences in multinational teams. In D. L. Shapiro (Ed.), 
Managing Multinational Teams: Global Perspectives Amsterdam: Elsevier.

Judge, T. A., Heller, D., \& Mount, M. K. 2002. Five-factor model of personality and job satisfaction: A meta-analysis. Journal of Applied Psychology, 530-541.

Judge, T. A., \& Ilies, R. 2002. Relationship of personality to performance motivation: A meta-analytic review. Journal of Applied Psychology, 87: 797807.

Katz, D., \& Kahn, R. 1966. The social psychology of organizations. New York: Wiley Co.

Katzenbach, J. R., \& Smith, D. K. 1993. The Discipline of Teams Harvard Business Review, March-April: 111-120.

Keller, R. T. 2001. Cross functional project groups in research and new product development diversity: communications, job stress, and outcomes. Academy of Management Journal, 44(3): 547-555.

Keller, R. T. 2006. Transformational Leadership, Initiating Structure, and Substitutes for Leadership: A Longitudinal Study of Research and Development Project Team Performance. Journal of Applied Psychology, 91(1): 202-210.

Kirkman, B. L., \& Rosen, B. 1999. Beyond Self-Management: Antecedents and Consequences of Team Empowerment. Academy of Management Journal, $42: 58$ - 74.

Kirkman, B. L., \& Shapiro, D. L. 2000. Understanding why team members won't share. An examination of factors related to employee receptivity to team-based rewards. Small Group Research, 31: 175-209.

Kirkman, B. L., \& Shapiro, D. L. 2001. The impact of cultural values on job satisfaction and organizational commitment in self-managing work teams: the mediating role of employee resistance. Academy of Management Journal, 44(3): 557-569.

Klein, K. J., Conn, A. B., Smith, B., \& Sorra, J. S. 2001. Is everyone in agreement? An exploration of within-group agreement in employee perceptions of the work environment. Journal of Applied Psychology, 86: 3-16.

Klein, K. J., Lim, B. C., Saltz, J. L., \& Mayer, D. M. 2004. How do they get there? An examination of the antecedents of centrality in team networks Academy of Management Journal, 47: 952-963.

Kogut, B., \& Zander, U. 1993. Knowledge of the firm and the evolutionary theory of the multinational corporation. Journal of International Business Studies, 24(4): 625-645.

Krenzel, G. A. 1999. Multinationale Arbeitsgruppen: Implikationen für die Führung. Wirtschaftsuniversität Wien.

Lagerstroem, K., \& Andersson, M. 2003. Creating and sharing knowledge within a transnational team - the development of a global business system. Journal of World Business, 38 84-95. 
Langfred, C. W. 2000. Work-group design and autonomy-a field study of the interaction between task interdependence and group autonomy. Small Group Research, 31: 54-70.

Langfred, C. W. 2005. Autonomy and performance in teams: The multilevel moderating effect of task interdependence. Journal of Management, 31: 513-529.

Lau, D. C., \& Murnighan, J. K. 1998. Demographic diversity and faultlines: the compositional dynamics of organizational groups. Academy of Management Review, 23,: 325-340.

Lovelace, K., Shapiro, D. L., \& Weingart, L. R. 2001. Maximizing crossfunctional new product teams' innovativeness and constraint adherence: a conflict communications perspective. Academy of Management Journal, 44: 779-793.

Luther, N. 2000. Integrity testing and job performance within high performance work teams: a short note. Journal of Business and Psychology, 15(1).

MacDonald, S., \& Hellgren, B. 2004. The Interview in International Business Research: Problems we would rather not talk about. In: R. MarschanPiekkari, \& C. Welch (Eds) Handbook of Qualitative Research Methods in International Business: 264-281. Cheltenham, UK: Edward Elgar.

Man, D. C., \& Lam, S. S. K. 2003. The effects of job complexity and autonomy on cohesiveness in collectivistic and individualistic work groups: A crosscultural analysis. Journal of Organizational Behavior, 24: 979- 1001.

Mann, R. D. 1959. A review of the relationship between personality and performance in small groups. Psychological Bulletin, 56: 241-270.

Marquardt, M. J., \& Horvath, L. 2001. Global Teams: How Top Multinationals Span Boundaries and Cultures with High-speed Teamwork. Palo Alto, CA: Davies-Black.

Marschan-Piekkari, R., \& Reis, C. 2004. Language and Languages in Crosscultural interviewing In: R. Marschan-Piekkari, \& C. Welch (Eds) Handbook of Qualitative Research Methods in International Business: 224-243. Cheltenham, UK: Edward Elgar.

Marschan-Piekkari, R., Welch, C., \& Tahvanainen, M. 2004. Interviewing in the Multinational Corporation: Challenges of the Organisational Context. In: R. Marschan-Piekkari, \& C. Welch (Eds) Handbook of Qualitative Research Methods in International Business: 244-263. Cheltenham, UK: Edward Elgar.

Marschan, R., Welch, D., \& Welch, L. 1997. Language: The forgotten factor in multinational management. European Management Journal, 15(5): 591598.

Mathieu, J. E., Gilson, L.L., \& Ruddy, T.M. 2006. Empowerment and Team Effectiveness: An Empirical Test of an Integrated Model. Journal of Applied Psychology, 91(1): 97-108. 
Matveev, A. V., \& Nelson, P. E. 2004. Cross Cultural Communication Competence and Multicultural Team Performance Perceptions of American and Russian Managers. International Journal of Cross Cultural Management, 4(2): 253-270.

Mayring, P. 2002. Eine Einführung in die qualitative Sozialforschung: Eine Anleitung zum qualitativen Denken. Weinheim, Basel.

McCracken, G. 1988. The long interview. Newbury Park, CA: Sage.

McCreery, J. K., \& Bloom, M. C. 2000. Teams: Design and implementation. In P. M Swamidass (Ed.), Encyclopedia of production \& manufacturing management. Boston,

\section{MA: Kluwer.}

Mendez, A. 2003. The coordination of globalized R\&D activities through project teams organization: an exploratory empirical study. Journal of World Business, 38: 96-109.

Miles, M., \& Huberman, M. 1994. Qualitative Data Analysis: An Expanded Sourcebook, 2nd edn, Beverly Hills, CA: Sage.

Milliken, F. J., \& Martins, L. L. 1996. Searching for common threads: Understanding the multiple effects of diversity in organizational groups. Academy of Management Review, 21: 402-433.

Morgeson, F. P., Reider, M. H., \& Campion, M. A. 2005. Selecting individuals in team settings: The importance of social skills, personality characteristics, and teamwork knowledge. Personnel Psychology, 58: 583-611.

Mueller, F., Procter, S., \& Buchanan, D. 2000. Teamworking in its context(s): Antecedents, nature and dimensions. Human Relations, 53: 1387-1424.

Neumann, G. A., \& Wright, J. 1999. Team effectiveness: beyond skills and cognitive ability. Journal of Applied Psychology, 84: 376-389.

Neumann, W. L. 2000. Social Research Methods. Qualitative and Quantitative Approaches. Boston: Allyn and Bacon.

Neyer, A. K. 2004. Multinational Teams in the European Commission and the European Parliament. Wirtschaftsuniversität Wien

Nutt, P. C. 2005. Comparing Public and Private Sector Decision-Making Practices. Journal of Public Administration Research and Theory, 16: 289318.

OIOS. 2006. Comprehensive Review of Governance and Oversight within the United Nations, Funds, Programmes and Specialised Agencies: United Nations.

Orsburn, J. D., Moran, L., Musselwhite, E., Zenger, J. H., \& Perrin, C. 1990. Self-directed work teams: The new American challenge. In J. S. Ott (Ed.), Classic readings in organizational behavior (2nd ed., pp. 272281). Fort Worth, TX: Harcourt Brace.

Osland, J., \& Osland, A. 2001. International qualitative research: an effective way to generate and verify cross-cultural theories. In: B. Toyne, Z.L. 
Martinez, \& R.A. Menger (Eds.) International Business Scholarship, Westport, CT: Quorum, pp. 198-214.

Patten, T. 1988. Team building, Part I: Designing the intervention. In: W. Brendan Reddy with Kaleel Jamison (Eds.), Team building pp. 15-24, Alexandria VA: NTL Institute for Applied Behavioral Sciences, San Diego University Associates.

Patton, M. 2002. Qualitative Research and Evaluation Methods. 3rd Edition, Sage Publications.

Pearce, C. L., \& Ensley, M.D. 2004. A reciprocal and longitudinal investigation of the innovation process: The central role of shared vision in product and process innovation teams (PPITs). Journal of Organizational Behavior, 25: 259-278.

Pelled, L., Eisenhardt, K., \& Xin, K. 1999. Exploring the Black Box: An Analysis of Work Group Diversity, Conflict, and Performance. Administrative Science Quarterly, 44: 1 - 28.

Peltokorpi, V. 2007. Intercultural communication patterns and tactics: Nordic expatriates in Japan. International Business Review, 16: 68-82.

Perry, J. L., \& Porter, L. W. 1982. Factors affecting the context for motivation in public organisations. Academy of Management Review, 7(1): 89-98.

Pettigrew, A. 1985. Contexutalist research: a natural way to link theory and practice. In: E.E. Lawler (ed) Doing research that is useful for theory and practice, San Francisco, CA: Jossey-Bass, pp. 222-248.

Pinfield, L. 1986. A field evaluation of perspectives on organizational decision making. Administrative Science Quarterly, 31: 365-388.

Polanyi, M. 1967. The tacit dimension. London, UK: Routledge \& Kegan.

Polzer, J., Crisp, C. B., Jarvenpaa, S. L., \& Kim, J. W. 2006. Extending the faultline model to geographically dispersed teams: how colocated subgroups can impair group functioning. Academy of Management Journal, 49(4): 679-692.

Polzer, J., Milton, L., \& Swann, W. 2002. Capitalizing on Diversity: Interpersonal Congruence in Small Work Groups. Administrative Science Quarterly, 47: 296-324.

Powell, G. N., Butterfield, D. A., \& Parent, J. D. 2002. Gender and managerial stereotypes: Have times changed? Journal of Applied Psychology, 28(2): 177-193.

Rainey, H., \& Bozeman, B. 2000. Comparing Public and Private Organisations: Empirical Research and the Power of the A Priori. Journal of Public Administration Research and Theory, 10: 776-469.

Randel, A. 2002. Identity salience: a moderator of the relationship between group gender composition and work group conflict. Journal of Organizational Behavior, 23: 749-766. 
Randel, A. 2003. The salience of culture in multinational teams and its relation to team citizenship behavior International Journal of Cross Cultural Management, 3(1): 27-44.

Randel, A., \& Jaussi, K. 2003. Functional Background Identity, Diversity, and Individual Performance in Cross-Functional Teams Academy of Management Journal, 46: 763 - 774.

Rasmussen, T., \& Jeppesen, H. 2006. Teamwork and associated psychological factors: a review. Work \& Stress, 20(2): 105-128.

Reagans, R., Zuckerman, E., \& McEvily, B. 2004. How to make the team: Social networks vs. demography as criteria for designing effective teams Administrative Science Quarterly, 49: 101-133.

Rentsch, J., \& Klimoski, R. 2001. Why do "great minds" think alike? Antecedents of team member schema agreement. Journal of Organizational Behavior, 22: 107-120.

Rousseau, D., \& Fried, Y. 2001. Location, location, location: contextualizing organisational research. Journal of Organizational Behavior, 22(1): 113.

Salas, E., Dickinson, T. L., Converse, S. A., \& Tannenbaum, S. I. 1992. Toward an understanding of team performance and training. In $R$. W. Swezey \& E. Salas (Eds.), Teams: Their training and performance (pp. 2-29). Norwood, NJ: Ablex.

Schein, E. 1984. Coming to a New Awareness of Organisational Culture. Sloan Management Review(Winter): 7.

Schein, E. 1985. Organizational culture and leadership. San Francisco: JosseyBass.

Schein, V. E. 1975. Relationships between sex role steretypes and requisite management characteristics among female managers. Journal of Applied Psychology (60): 340-344.

Schippers, M., Den Hartog, D., \& Koopman, P. 2007. Reflexivity in Teams: A Measure and Correlates. Applied Psychology: An international review, 56(2): 189-211.

Schippers, M., Den Hartog, D., Koopman, P., \& Wienk, J. 2003. Diversity and team outcomes: the moderating effects of outcome interdependence and group longevity and the mediating effect of reflectivity. Journal of Organizational Behavior, 24: 779-802.

Schneider, B. 1985. Interactional psychology and organizational behavior. In Research in OrganizationalBehavior, Vol. 5, Staw, B. Cummings LL. (eds). JAI Press: Greenwich, CT; 1-31.

Schwab, D. P. 1999. Research methods for organizational studies. Hillsdale, NJ: Erlbaum.

Schwartz, S. H. 1994. Beyond Individualism/Collectivism. New Cultural Dimensions of Values. In: U.Kim et al. (Eds.): Individualism and Collectivism: Theory, Methods and Applications. London: Sage. 
Sewell, G., \& Barker, J. R. 2006. Coercion versus care: using irony to make sense of organisational surveillance. Academy of Management Review, 31(4): 934-961.

Sparrowe, R. T., \& Liden, R. C. 1997. Process and Structure in Leader-Member Exchange. Academy of Management Journal, 22(2): 522-552.

Sprigg, C., Jackson, P., \& Parker, S. 2000. Production teamworking: The importance of interdependence and autonomy for employee strain and satisfaction. Human Relations, 53: 1519-1543.

Stake, $\mathrm{R}$. E. 1995. The art of case study research. Thousand Oaks: Sage Publications.

Stewart, G. L. 2006. A Meta-Analytic Review of Relationships Between Team Design Features and Team Performance. Journal of Management, 32(1): 29-54.

Stock, R. 2004. Drivers of Team Performance: What do we know and what have we still to learn? . Schmalenbach Business Review, 56: 274-206.

Strauss, A., \& Corbin, J. 1994. Grounded Theory Methodology: An overview. In: N.K. Denzin \& Y.S. Lincoln (Eds.), Handbook of Qualitative Research. Thousand Oaks: Sage Publications, p 273-285.

Sundstrom, E., DeMeuse, K. P., \& Futrell, D. 1990. Work teams: applications and effectiveness. American Psychologist, 45: 120-133.

Swann, W. B., Polzer, J., Seyle, D., \& S., K. 2004. Finding value in diversity: verification of personal and social self-views in diverse groups. Academy of Management Review, 29: 9-27.

Tajfel, H., \& Turner, J. 1986. The social identity theory of intergroup behavior. In W. A. S. Worchel (Ed.), Psychology and intergroup relations. Chicago: Nelson-Hall.

Triandis, Kurowski, \& Gelfand. 1994. Workplace diversity. In Handbook of Industrial and Organizational Psychology, Vol. 4, ed. HC Triandis, MD Dunnette, LM Hough, pp. 769-827. Palo Alto, CA: Consult. Psychol. Press. 2nd ed.

Trompenaars, F., \& Hampden-Turner, C. 1997. Riding the waves of culture: Understanding Cultural Diversity in Business (2 ed.). London.

Turner, B. 1981. Some practical aspects of qualitative data analyses: one way of organizing the cognitive process associated with the generation of grounded theory. Quality and Quantity, 15: 225-247.

UN. Charter of the United Nations.

Vallaster, A. 2005. Cultural Diversity and Its Impact on Social Interactive Processes Implications from an Empirical Study. International Journal of Cross Cultural Management 5(2): 139-163.

Van de Vijver, F., \& Tanzer, N. K. 1997. Bias and equivalent in cross-cultural assessment: an overview. European Review of Applied Psychology, 47(4): 263-279. 
Van der Vegt, G., \& Bunderson, J. S. 2005. Learning and performance in multidisciplinary teams: the importance of collective team identification. Academy of Management Journal, 48(3): 532-547.

Van der Vegt, G., Emans, B., \& Van de Vliert, E. 2001. Patterns of interdependence in work teams: A two level Investigation of the relations with job and team satisfaction Personnel Psychology, 54: 51- 69.

Van der Vegt, G., \& Van de Vliert, E. 2000. Team members' affective responses to patterns of intragroup interdependence and job complexity. Journal of Management, 26: 633-645.

Van der Vegt, G., Van de Vliert, E., \& Oosterhof, A. 2003. Informational dissimilarity and organizational citizenship behavior: The role of intrateam interdependence and team identification Academy of Management Journal, 46: 715-727.

Van Emmerik, H., Lambooy, M., \& Sanders, K. 2002. Differential effects of individual-linked and team-level status allocation on professionals' job performance. Small Group Research, 33: 702-717.

Van Knippenberg, D., \& Schippers M. 2007. Work Group Diversity. Annual Review of Psychology, 58: 515-541.

Van Knippenberg, D., de Dreu, C., \& Homann, A. 2004. Work group diversity and group performance: an integrative model and research agenda. Journal of Applied Psychology, 89: 1008-1022.

Van Maanen, J. 1983. Reclaiming qualitative methods for organizational research: a preface. In J. V. Maanen (Ed.), Qualitative Methodology: 9-18. Beverly Hills: CA: Sage.

Wagner, J. A. 1995. Studies of individualism-collectivism: Effects on cooperation in groups. Academy of Management Journal, 38: 152-172.

Wall, T. D., Kemp, N. J., Jackson, P. R., \& Clegg, C. W. 1986. Outcomes of autonomous workgroups: A long-term field experiment. Academy of Management Journal, 29: 280-304.

Ward, C. 1996. Acculturation. In D. Landis, \& R. S. Bhagat (Eds.), Handbook of Intercultural Training: 124-147. Thousand Oaks

CA: Sage.

Webber, S., \& Donahue, L. 2001. Impact of highly and less job-related diversity on work group cohesion and performance: a meta-analysis. Journal of Management, 27: 141-163.

Weber, M. 1922. Wirtschaft und Gesellschaft, Grundriß der verstehenden Soziologie: Secher, H.P. (Ed.).

Weber, M. 1962. Basic concepts in sociology. London: Peter Owen.

Weber, M. 1978. Economy and Society. Los Angeles: University of California Press.

Weick, K. E. 2001. Making Sense of the Organization. Oxford, UK: Blackwell. 
Welch, D., Welch, L., \& Piekkari, R. 2005. Speaking in Tongues. The importance of language in international management processes. Int.Studies of Management and Organisations, 35(1): 10-27.

Wilkinson, I., \& Young, L. 2004. Improvisation and Adaptation in International Business Research Interviews In: R. Marschan-Piekkari, \& C. Welch (Eds) Handbook of Qualitative Research Methods in International Business: 207-223. Cheltenham, UK: Edward Elgar.

Williams, \& O'Reilly. 1998. Demography and diversity in organizations: a review of 40 years of research. In: Research in Organizational Behavior, ed. Staw, Cummings, p77-140.

Wilson, W. 1887. The study of administration. Political Science quarterly(2): 197-222.

Yan, A., \& Louis, M. R. 1999. The Migration of Organizational Functions to the Work Unit Level: Buffering, Spanning, and Bringing Up Boundaries. Human Relations, 52(25): 25-47.

Yeung, H. 1995. Qualitative Personal Interviews in International Business Research: Some Lessons from a Study of Hong Kong Transnational Corporations. International Business Review, 4(3): 313-339.

Yin, R. 1984. Case study research. Beverly Hills, CA: Sage Publications.

Yin, R. 1994. Case Study Research: Design and Methods (2nd ed.). Thousand Oaks, CA: Sage.

Yukl, G. A. 2002. Leadership in Organizations. New York: Prentice-Hall.

Zohar, D. 2002. The effects of leadership dimensions, safety climate, and assigned priorities on minor injuries in work groups. Journal of Organizational Behavior, 23: 75-92. 


\section{Appendices}

\section{Appendix 1: List of articles reviewed}

\begin{tabular}{|c|c|c|c|c|}
\hline Article & Sector & $\begin{array}{l}\text { quantitative/ } \\
\text { qualitative }\end{array}$ & Term & Definition of term in article \\
\hline $\begin{array}{l}\text { Alper, Tjosvold, } \\
\text { \& Law }(2000)\end{array}$ & private & quantitative & SMWT & $\mathbf{N} / \mathbf{A}$ \\
\hline $\begin{array}{l}\text { Ambos, } \\
\text { Schlegelmilch } \\
\text { (2004) }\end{array}$ & private & quantitative & R\&D team & $\begin{array}{l}\text { "The teams are international, as they } \\
\text { span distances among internationally } \\
\text { dispersed R\&D units, integrative, as } \\
\text { they are established to coordinate } \\
\text { work among individual actors, and } \\
\text { technology oriented in focus, as they } \\
\text { aim to integrate the firms' interna- } \\
\text { tional R\&D efforts." }\end{array}$ \\
\hline $\begin{array}{l}\text { Ambrosini, } \\
\text { Bowman, \& } \\
\text { Burton-Taylor } \\
(2007)\end{array}$ & private & qualitative & team & $\mathbf{N} / \mathbf{A}$ \\
\hline $\begin{array}{l}\text { Armstrong- } \\
\text { Stassen, Wagar, } \\
\text { \& Cattaneo } \\
(2004)\end{array}$ & public & quantitative & team/group & $\mathbf{N} / \mathbf{A}$ \\
\hline $\begin{array}{l}\text { Ayoko, Haertcl } \\
(2003)\end{array}$ & private & qualitative & team & $\begin{array}{l}\text { "[...] Teams comprise members from } \\
\text { different ethnic, racial, cultural, or } \\
\text { national backgrounds. We use the } \\
\text { term interchangeably with the term } \\
\text { diverse workgroups." }\end{array}$ \\
\hline Barinaga (2007) & private & qualitative & team & N/A \\
\hline $\begin{array}{l}\text { Barrick, Stewart, } \\
\text { Neubert, \& } \\
\text { Mount (1998) }\end{array}$ & private & quantitative & team & acc. to Guzzo \& Dickson (1996) \\
\hline $\begin{array}{l}\text { Bishop \& Scott } \\
(2000)\end{array}$ & private & quantitative & SDWT & $\begin{array}{l}\text { acc. to Wall, Kemp, Jackson, \& Clegg } \\
\text { (1986) }\end{array}$ \\
\hline $\begin{array}{l}\text { Bishop, Scott \& } \\
\text { Burroughs } \\
(2000)\end{array}$ & private & quantitative & team & 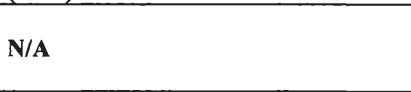 \\
\hline $\begin{array}{l}\text { Bowers, } \\
\text { Pharmer, \& } \\
\text { Salas }(2000)\end{array}$ & meta & quantitative & team & $\mathbf{N} / \mathbf{A}$ \\
\hline $\begin{array}{l}\text { Bricf, Umphress, } \\
\text { Dietz, Butz, } \\
\text { Burrows, \& } \\
\text { Scholten (2005) }\end{array}$ & meta & quantitative & group & $\mathbf{N} / \mathbf{A}$ \\
\hline $\begin{array}{l}\text { Bunderson } \\
(2003)\end{array}$ & private & quantitative & group & $\mathbf{N} / \mathbf{A}$ \\
\hline $\begin{array}{l}\text { Burke, Stagl, } \\
\text { Salas, Pierce, \& } \\
\text { Kendall (2006) }\end{array}$ & theoretical & theoretical & team & $\mathbf{N} / \mathbf{A}$ \\
\hline $\begin{array}{l}\text { Cannon, \& } \\
\text { Edmondson } \\
\text { (2001) }\end{array}$ & private & quantitative & $\begin{array}{l}\text { work } \\
\text { group }\end{array}$ & $\mathbf{N} / \mathbf{A}$ \\
\hline $\begin{array}{l}\text { Carless \& De } \\
\text { Paola (2000) }\end{array}$ & public & quantitative & team & $\mathbf{N} / \mathbf{A}$ \\
\hline Chang, Tharenou & private & qualitative & work & $\mathbf{N} / \mathbf{A}$ \\
\hline
\end{tabular}




\begin{tabular}{|c|c|c|c|c|}
\hline$(2004)$ & & & group & \\
\hline $\begin{array}{l}\text { Chansler, Swa- } \\
\text { midass, \& Cam- } \\
\text { mann (2003) }\end{array}$ & privatc & quantitative & SMWT & acc. to McCrcery, \& Bloom (2000) \\
\hline $\begin{array}{l}\text { Chatman, \& } \\
\text { O'Rcilly (2004) }\end{array}$ & private & quantitative & team & N/A \\
\hline $\begin{array}{l}\text { Chattopadhyay } \\
\text { (1999) }\end{array}$ & privatc & quantitative & group & acc. to Hackman (1983) \\
\hline $\begin{array}{l}\text { Chattopadhyay, } \\
\text { Tluchowska, \& } \\
\text { Gcorge (2004) }\end{array}$ & theoretical & N/A & group & acc. to Hackman (1987) \\
\hline $\begin{array}{l}\text { Chen, Kirkman, } \\
\text { Kanfer, Allen, \& } \\
\text { Rosen (2007a) }\end{array}$ & privatc & quantitative & team & N/A \\
\hline $\begin{array}{l}\text { Chen, Lam, \& } \\
\text { Zhong (2007b) }\end{array}$ & private & quantitative & team & $\mathbf{N} / \mathbf{A}$ \\
\hline Chevricr (2003) & private & qualitative & $\begin{array}{l}\text { project } \\
\text { group/team }\end{array}$ & N/A \\
\hline Choi (2002) & theoretical & theoretical & teams & $\mathbf{N} / \mathbf{A}$ \\
\hline $\begin{array}{l}\text { Colquitt, Noe, \& } \\
\text { Jackson (2002) }\end{array}$ & private & quantitative & team & $\begin{array}{l}\text { acc. to Cohen \& Bailcy; Guzzo \& } \\
\text { Dickson, Sundstrom, DeMcuse, \& } \\
\text { Futrell }\end{array}$ \\
\hline $\begin{array}{l}\text { Cross, Yan \& } \\
\text { Louis }(2000)\end{array}$ & privatc & qualitative & team & ( \\
\hline $\begin{array}{l}\text { De Dreu \& West } \\
(2001)\end{array}$ & private & quantitative & team & $\mathbf{N} / \mathbf{A}$ \\
\hline De Drcu (2006) & private & quantitative & tcam & N/A \\
\hline $\begin{array}{l}\text { De Dreu, Wcin- } \\
\text { gart }(2003)\end{array}$ & meta & quantitative & team & $\mathbf{N} / \mathbf{A}$ \\
\hline $\begin{array}{l}\text { Driskcll, Good- } \\
\text { win, Salas, } \\
\text { O'Shca (2006) }\end{array}$ & theoretical & theoretical & team & $\mathbf{N}$ \\
\hline $\begin{array}{l}\text { Earlcy, Mosa- } \\
\text { kowski }(2000)\end{array}$ & privatc & qualitative & team & N/A \\
\hline $\begin{array}{l}\text { Eby, Adams, } \\
\text { Russel, \& Gaby } \\
(2000)\end{array}$ & private & quantitative & team & N/A \\
\hline $\begin{array}{l}\text { Ellemers, de } \\
\text { Gilder, \& Van } \\
\text { den Heuvel } \\
(1998)\end{array}$ & private & quantitative & team & N/A \\
\hline $\begin{array}{l}\text { Elloy, Terpen- } \\
\text { ing, \& Kohls } \\
(2001)\end{array}$ & privatc & quantitative & team & N/A \\
\hline Ely (2004) & privatc & quantitative & group & N/A \\
\hline $\begin{array}{l}\text { Ely, Thomas } \\
(2001)\end{array}$ & private & qualitative & group & $\mathbf{N} / \mathbf{A}$ \\
\hline $\begin{array}{l}\text { English, Griffith, } \\
\text { \& Steelman } \\
\text { (2004) }\end{array}$ & privatc & quantitative & team & N/A \\
\hline $\begin{array}{l}\text { Flynn, Chatman, } \\
\text { \& Spataro (2001) }\end{array}$ & private & quantitative & team & $\mathbf{N} / \mathbf{A}$ \\
\hline $\begin{array}{l}\text { Garcia-Pricto, } \\
\text { Bellard, Schnei- } \\
\text { der (2003) }\end{array}$ & theoretical & theoretical & team & N/A \\
\hline $\begin{array}{l}\text { Gibson, Ver- } \\
\text { meulen }(2003)\end{array}$ & privatc & quantitative & group/team & N/A \\
\hline Gibson, Zcllmer- & private & qualitative + & team & yes, whole article is about team and its \\
\hline
\end{tabular}




\begin{tabular}{|c|c|c|c|c|}
\hline Bruhn (2001) & & quantiative & & meaning \\
\hline $\begin{array}{l}\text { Gilson \& Shalley } \\
\text { (2004) }\end{array}$ & private & quantitative & team & N/A \\
\hline $\begin{array}{l}\text { Gilson, Mathicu, } \\
\text { Shalley, \& } \\
\text { Ruddy (2005) }\end{array}$ & private & quantitative & team & $\begin{array}{l}\text { "[...] usually highly trained on the } \\
\text { tasks they perform, as well as on how } \\
\text { to handle a complex and divers cus- } \\
\text { tomer base" }\end{array}$ \\
\hline $\begin{array}{l}\text { Goodall, Roberts } \\
\text { (2003) }\end{array}$ & private & qualitative & team & ( \\
\hline $\begin{array}{l}\text { Harrison, Price, } \\
\text { \& Bell (1998) }\end{array}$ & private & quantitative & group & $\mathbf{N} / \mathbf{A}$ \\
\hline $\begin{array}{l}\text { Hirschfeld, Jor- } \\
\text { dan, Feild, Giles, } \\
\text { \& Armenakis } \\
\text { (2006) }\end{array}$ & public & quantitative & team & $\mathbf{N} / \mathbf{A}$ \\
\hline $\begin{array}{l}\text { Hobman, Bordia, } \\
\text { \& Gallois (2003) }\end{array}$ & public & quantitative & team & N/A \\
\hline $\begin{array}{l}\text { Hoegl, Parbo- } \\
\text { teeah (2003)) }\end{array}$ & private & quantitative & team & $\begin{array}{l}\text { " [...] } 6 \text { Teamwork quality facets: open } \\
\text { sharing of relevant information, coor- } \\
\text { dinating team tasks, utilising all team } \\
\text { members' knowledge and expertise, } \\
\text { mutually supporting each other, exert- } \\
\text { ing all efforts on the team task, and } \\
\text { promoting team spirit and cohesion." }\end{array}$ \\
\hline $\begin{array}{l}\text { Jackson, Joshi, \& } \\
\text { Erhardt (2004) }\end{array}$ & private & quantitative & team & N/A \\
\hline $\begin{array}{l}\text { Jackson, Joshi, \& } \\
\text { Erhardt (2003) }\end{array}$ & theoretical & N/A & team & \\
\hline $\begin{array}{l}\text { Jehn, \& Bez- } \\
\text { rukova (2004) }\end{array}$ & private & quantitative & group & N/A \\
\hline $\begin{array}{l}\text { Jehn, Northcraft, } \\
\text { \& Neale (1999) }\end{array}$ & private & quantitative & group & $\mathbf{N} / \mathbf{A}$ \\
\hline Joshi (2006) & theoretical & N/A & team & N/A \\
\hline $\begin{array}{l}\text { Joshi et al. } \\
(2002)\end{array}$ & private & quantitative & team & $\mathbf{N} / \mathbf{A}$ \\
\hline Keller (2001) & private & quantitative & group & N/A \\
\hline Keller (2006) & private & quantitative & team & N/A \\
\hline $\begin{array}{l}\text { Kirkman, Rosen } \\
\text { (1999) }\end{array}$ & private & quantitative & team & acc. to Hackman (1987) \\
\hline $\begin{array}{l}\text { Kirkman, } \\
\text { Shapiro }(2000)\end{array}$ & private & quantitative & team & $\begin{array}{l}\text { acc. to Cohen \& Bailey; Sundstrom, } \\
\text { DeMeuse, \& Futrell }\end{array}$ \\
\hline $\begin{array}{l}\text { Kirkman, } \\
\text { Shapiro (2001) }\end{array}$ & private & quantitative & SMWT & $\begin{array}{l}\text { Self-managing work teams are defined } \\
\text { as teams whose members do the fol- } \\
\text { lowing: manage themselves, assign } \\
\text { jobs, plan and schedule work, make } \\
\text { production- or service-related deci- } \\
\text { sions, and take action on problems } \\
\text { (Wellins et al., 1990). }\end{array}$ \\
\hline $\begin{array}{l}\text { Klein, Conn, } \\
\text { Smith, \& Sorra } \\
\text { (2001) }\end{array}$ & private & quantitative & group & N/A \\
\hline $\begin{array}{l}\text { Klein, Lim, } \\
\text { Saltz, \& Mayer } \\
\text { (2004) }\end{array}$ & public & quantitative & team & N/A \\
\hline $\begin{array}{l}\text { Lagerstroem, } \\
\text { Andersson } \\
(2003)\end{array}$ & private & qualitative & teams & $\begin{array}{l}\text { The transnational team is a cross- } \\
\text { border organizational unit composed } \\
\text { of individuals of different na- } \\
\text { tionalaities, working in different cul- }\end{array}$ \\
\hline
\end{tabular}




\begin{tabular}{|c|c|c|c|c|}
\hline & & & & $\begin{array}{l}\text { tures, business units, and functions, } \\
\text { thereby possessing specialized knowl- } \\
\text { edge for solving a common global task } \\
\text { in the MNC (Gupta \& Govindarajan, } \\
\text { 2001; Schweiger, 1998) }\end{array}$ \\
\hline Langfred (2000) & private & quantitative & team & acc. to Guzzo \& Dickson (1996) \\
\hline Langfred (2005) & private & quantitative & teams & N/A \\
\hline $\begin{array}{l}\text { Lau, Murnighan } \\
\text { (1998) }\end{array}$ & theoretical & theoretical & $\begin{array}{l}\text { groups (but } \\
\text { not real } \\
\text { ones) } \\
\end{array}$ & N/A \\
\hline $\begin{array}{l}\text { Lovclace, } \\
\text { Shapiro, \& } \\
\text { Weingart (2001) }\end{array}$ & private & quantitative & team & N/A \\
\hline Luther (2000) & private & quantitative & team & N/A \\
\hline $\begin{array}{l}\text { Man, \& Lam } \\
(2003)\end{array}$ & privatc & quantitative & $\begin{array}{l}\text { work } \\
\text { group, } \\
\text { team }\end{array}$ & N/A \\
\hline $\begin{array}{l}\text { Mathicu, Gilson, } \\
\text { \& Ruddy (2006) }\end{array}$ & private & quantitative & teams & acc. to Sundstrom (1999) \\
\hline $\begin{array}{l}\text { Matveev, Nelson } \\
(2004)\end{array}$ & private & quantitative & team & $\begin{array}{l}\text { "Multicultural teams are task-oriented } \\
\text { groups consisting of people of different } \\
\text { national cultures (Marquardt and } \\
\text { Horvath, 2001)" }\end{array}$ \\
\hline Mendez (2003) & privatc & theoretical & teams & N/A \\
\hline $\begin{array}{l}\text { Morgeson, Rei- } \\
\text { der, \& Campion } \\
(2005)\end{array}$ & private & quantitative & team & N/A \\
\hline $\begin{array}{l}\text { Mucller, Procter, } \\
\text { Buchanan }(2000)\end{array}$ & theoretical & theoretical & team & N/A \\
\hline $\begin{array}{l}\text { Neumann, } \\
\text { Wright (1999) }\end{array}$ & private & quantitative & team & $\begin{array}{l}\text { "Contrary to groups, teams consist of } \\
\text { two or more individuals that can be } \\
\text { characterized by high task interde- } \\
\text { pendence, high role differentiation, } \\
\text { high task differentiation, and distrib- } \\
\text { uted expertise (Salas et al., 1992; } \\
\text { Sundstrom, de Meuse, \& Futrell, } \\
\text { 1990). As a result, each team member } \\
\text { has specialized knowledge and skills } \\
\text { and must coordinate and interact with } \\
\text { other team members sequentially or } \\
\text { simultaneously (Dickinson \& } \\
\text { Mclntyre, 1997). In summary, team } \\
\text { members interact frequently with each } \\
\text { other and are interdependent, creating } \\
\text { unique interpersonal demands on team } \\
\text { members to accomplish task perform- } \\
\text { ance." }\end{array}$ \\
\hline $\begin{array}{l}\text { Pearce, \& Enslcy } \\
(2004)\end{array}$ & private & quantitative & team & N/A \\
\hline $\begin{array}{l}\text { Pelled, Eisen- } \\
\text { hardt, \& Xin } \\
\text { (1999) }\end{array}$ & privatc & quantitative & group & N/A \\
\hline $\begin{array}{l}\text { Polzer, Milton, } \\
\text { \& Swann }(2002)\end{array}$ & private & quantitative & group & N/A \\
\hline Randel (2002) & privatc & quantitative & group & N/A \\
\hline Randel (2003) & private & quantitative & team & N/A \\
\hline $\begin{array}{l}\text { Randel, Jaussi } \\
\text { (2003) }\end{array}$ & private & quantitative & team & N/A \\
\hline
\end{tabular}




\begin{tabular}{|c|c|c|c|c|}
\hline $\begin{array}{l}\text { Reagans, Zuck- } \\
\text { ermann, } \& \text { McE- } \\
\text { vily (2004) }\end{array}$ & private & quantitative & team & N/A \\
\hline $\begin{array}{l}\text { Rentch, \& } \\
\text { Klimoski (2001) }\end{array}$ & public & quantitative & team & acc. to Sundstrom (1999) \\
\hline $\begin{array}{l}\text { Schippers et. al. } \\
(2003)\end{array}$ & private & quantitative & team & acc. to Hackman (1987) \\
\hline $\begin{array}{l}\text { Schippers, Den } \\
\text { Hartog \& } \\
\text { Koopman (2007) }\end{array}$ & private & quantitative & team & N/A \\
\hline $\begin{array}{l}\text { Sprigg, Jackson, } \\
\text { \& Parker }(2000)\end{array}$ & private & quantitative & team & N/A \\
\hline Stewart (2006) & meta & quantitative & team & \\
\hline $\begin{array}{l}\text { Swann, Polzer, } \\
\text { Seylc, \& Ko } \\
\text { (2004) }\end{array}$ & theoretical & N/A & group & \\
\hline Vallaster (2005) & private & qualitative & team/group & N/A \\
\hline $\begin{array}{l}\text { Van der Vegt \& } \\
\text { Bunderson } \\
(2005)\end{array}$ & private & quantitative & team & N/A \\
\hline $\begin{array}{l}\text { Van der Vegt, \& } \\
\text { Van de Vliert } \\
(2000)\end{array}$ & private & quantitative & team & $\mathbf{N} / \mathbf{A}$ \\
\hline $\begin{array}{l}\text { Van der Vegt, } \\
\text { Emans, \& Van } \\
\text { dc Vliert (2001) }\end{array}$ & private & quantitative & team & $\begin{array}{l}\text { acc. to Hackman; Sundstrom, De- } \\
\text { Meuse, \& Futrell }\end{array}$ \\
\hline $\begin{array}{l}\text { Van der Vegt, } \\
\text { Van de Vliert, \& } \\
\text { Oosterhof (2003) }\end{array}$ & private & quantitative & team & N/A \\
\hline $\begin{array}{l}\text { Van Emmerik, } \\
\text { Lambooy, \& } \\
\text { Sanders (2002) }\end{array}$ & public & quantitative & team & N/A \\
\hline $\begin{array}{l}\text { Van } \\
\text { Knippenberg, De } \\
\text { Dreu, \& Homan } \\
(2004)\end{array}$ & theoretical & theoretical & group & N/A \\
\hline $\begin{array}{l}\text { Webber, Dona- } \\
\text { hue }(2001)\end{array}$ & meta & quantitative & group & N/A \\
\hline Zohar (2002) & private & quantitative & group & $\mathbf{N} / \mathbf{A}$ \\
\hline
\end{tabular}




\section{Appendix 2: Interview guide}

\section{Introduction}

Thank you for taking the time

Brief introduction to the research project and its goal

Explanation why it is important to conduct the interview

Reassurance that data are confidential

\section{Interview}

Describe your work environment

- do you work in a team/group. If yes

- how do you define a team/group

- who do you work with, which countries are they from

- is there a related story you might want to tell

- how long have you worked together

What is the role of management

What is the structure of the organisation

How do you experience red tape

What would you change if you could

\section{End}

Any other comments

Administration of demographic elements

Exchange of documentation etc. mentioned in interview

Referral to other interview partners

Thankyou 


\section{Appendix 3: Notes on interview situation}

To be completed by the interviewer immediately after the interview

\section{Background of the interviewee}

Name:

Job title:

Educational background:

Organisational level:

Tenure with organisation:

Foreign assignments:

Number of foreign languages:

\section{Interview situation}

Where did the interview take place? Short description of location:

Did the interviewee seem to understand the questions?

Good 0 fair 0 poor 0

How was the interviewee cooperating during the interview?

Good 0 fair 0 poor 0

Did the interviewee refer to documents?

Yes 0 no 0

Other comments regarding the interviewee and/or interview situation: 


\section{Forschungsergebnisse der Wirtschaftsuniversität Wien}

Herausgeber: Wirtschaftsuniversităt Wien vertreten durch a.o. Univ. Prof. Dr. Barbara Sporn

Band 1 Stefan Felder: Frequenzallokation in der Telekommunikation. Ökonomische Analyse der Vergabe von Frequenzen unter besonderer Berücksichtigung der UMTS-Auktionen. 2004.

Band 2 Thomas Haller: Marketing im liberalisierten Strommarkt. Kommunikation und Produktplanung im Privatkundenmarkt. 2005.

Band 3 Alexander Stremitzer: Agency Theory: Methodology, Analysis. A Structured Approach to Writing Contracts. 2005.

Band 4 Günther Sedlacek: Analyse der Studiendauer und des Studienabbruch-Risikos. Unter Verwendung der statistischen Methoden der Ereignisanalyse. 2004.

Band 5 Monika Knassmüller: Unternehmensleitbilder im Vergleich. Sinn- und Bedeutungsrahmen deutschsprachiger Unternehmensleitbilder - Versuch einer empirischen (Re-)Konstruktion. 2005.

Band 6 Matthias Fink: Erfolgsfaktor Selbstverpflichtung bei vertrauensbasierten Kooperationen. Mit einem empirischen Befund. 2005.

Band 7 Michael Gerhard Kraft: Ökonomie zwischen Wissenschaft und Ethik. Eine dogmenhistorische Untersuchung von Léon M.E. Walras bis Milton Friedman. 2005.

Band 8 Ingrid Zechmeister: Mental Health Care Financing in the Process of Change. Challenges and Approaches for Austria. 2005.

Band 9 Sarah Meisenberger: Strukturierte Organisationen und Wissen. 2005.

Band 10 Anne-Katrin Neyer: Multinational teams in the European Commission and the European Parliament. 2005.

Band 11 Birgit Trukeschitz: Im Dienst Sozialer Dienste. Ökonomische Analyse der Beschäftigung in sozialen Dienstleistungseinrichtungen des Nonprofit Sektors. 2006

Band 12 Marcus Kölling: Interkulturelles Wissensmanagement. Deutschland Ost und West. 2006.

Band 13 Ulrich Berger: The Economics of Two-way Interconnection. 2006.

Band 14 Susanne Guth: Interoperability of DRM Systems. Exchanging and Processing XML-based Rights Expressions. 2006.

Band 15 Bernhard Klement: Ökonomische Kriterien und Anreizmechanismen für eine effiziente Förderung von industrieller Forschung und Innovation. Mit einer empirischen Quantifizierung der Hebeleffekte von F\&E-Förderinstrumenten in Österreich. 2006.

Band 16 Markus Imgrund: Wege aus der Insolvenz. Eine Analyse der Forttührung und Sanierung insolventer Klein- und Mittelbetriebe unter besonderer Berücksichtigung des Konfigurationsansatzes. 2007.

Band 17 Nicolas Knotzer: Product Recommendations in E-Commerce Retailing Applications. 2008.

Band 18 Astrid Dickinger: Perceived Quality of Mobile Services. A Segment-Specific Analysis. 2007.

Band 19 Nadine Wiedermann-Ondrej: Hybride Finanzierungsinstrumente in der nationalen und internationalen Besteuerung der USA. 2008.

Band 20 Helmut Sorger: Entscheidungsorientiertes Risikomanagement in der Industrieunternehmung. 2008.

Band 21 Martin Rietsch: Messung und Analyse des ökonomischen Wechselkursrisikos aus Unternehmenssicht: Ein stochastischer Simulationsansatz. 2008. 
Band 22 Hans Christian Mantler: Makroökonomische Effizienz des Finanzsektors. Herleitung eines theoretischen Modells und Schătzung der Wachstumsimplikationen für die Marktwirtschaften und Transformationsőkonomien Europas. 2008.

Band 23 Youri Tacoun: La théorie de la valeur de Christian von Ehrenfels. 2008.

Band 24 Monika Koller: Longitudinale Betrachtung der Kognitiven Dissonanz. Eine Tagebuchstudie zur Reiseentscheidung. 2008.

Band 25 Marcus Scheiblecker: The Austrian Business Cycle in the European Context. 2008.

Band 26 Aida Numic: Multinational Teams in European and American Companies. 2008.

Band 27 Ulrike Bauernfeind: User Satisfaction with Personalised Internet Applications. 2008.

Band 28 Reinhold Schodl: Systematische Analyse und Bewertung komplexer Supply Chain Prozesse bei dynamischer Festlegung des Auftragsentkopplungspunkts. 2008.

Band 29 Bianca Gusenbauer: Őffentlich-private Finanzierung von Infrastruktur in Entwicklungsländern und deren Beitrag zur Armutsreduktion. Fallstudien in Vietnam und auf den Philippinen. 2009.

Band 30 Elisabeth Salomon: Hybrides Management in sino-österreichischen Joint Ventures in China aus österreichischer Perspektive. 2009.

Band 31 Katharina Mader: Gender Budgeting: Ein emanzipatorisches, finanzpolitisches und demokratiepolitisches Instrument. 2009.

Band 32 Michael Weber: Die Generierung von Empfehlungen für zwischenbetriebliche Transaktionen als gesamtwirtschaftliche Infrastrukturleistung. 2009.

Band 33 Lisa Gimpl-Heersink: Joint Pricing and Inventory Control under Reference Price Effects. 2009.

Band 34 Stefan A. Zopf: Analyse der Potentiale und Effekte des Ingredient Brandings und dessen Einfluss auf die Markenstärke. 2009.

Band 35 Dagmar Kiefer: Multicultural Work in Five United Nations Organisations. An Austrian Perspective. 2009.

www.peterlang.de 


\section{Multinational Teams in European and American Companies}

Frankfurt am Main, Berlin, Bern, Bruxelles, New York, Oxford, Wien, 2008. 234 pp., 19 tab.

Forschungsergebnisse der Wirtschaftsuniversität Wien. Vol. 26

Edited by: Wirtschaftsuniversität Wien

ISBN 978-3-631-57767-7 · pb. € 42.50*

Incorporating team context into research and practice concerning team effectiveness in multinational organizations still remains an ongoing challenge. This book aims at drawing the attention of researchers and practitioners towards the importance of various 'layers' of context on multinational teams and developing an empirically derived framework for multinational team functioning in business organisations. It shows how companies make use of these teams and how these teams contribute to competitive advantage. The study has been conducted in an Austrian, a German, and an American company. It reveals that if managed appropriately, these teams reduce the complexity of operations by facilitating the creation and transfer of explicit and tacit knowledge and by transferring appropriate dimensions of headquarter corporate culture between geographically dispersed business units.

Contents: Literature review on multinational teams - A purposeful approach to the interview and data analysis method in studying MNTs in European and American companies - Empirical findings from VA TECH - Empirical findings from Henkel Central Eastern Europe (CEE) - Empirical findings from K\&M International Summary and the conceptual framework for MNTs in business organisations

Frankfurt am Main - Berlin - Bern - Bruxelles - New York · Oxford - Wien

Distribution: Verlag Peter Lang AG

Moosstr. 1, $\mathrm{CH}-2542$ Pieterlen

Telefax 0041 (0) $32 / 3761727$

*The $€$-price includes German tax rate Prices are subject to change without notice

Homepage http://www.peterlang.de 
Dagmar Kiefer - 978-3-631-75389-7

Downloaded from PubFactory at 01/11/2019 05:36:21AM

via free access 
Dagmar Kiefer - 978-3-631-75389-7

Downloaded from PubFactory at 01/11/2019 05:36:21AM

via free access 